\title{
Studying the Health of the Bitcoin Ecosystem in GitHub
}

by

\section{Khadija Osman}

\author{
A Thesis submitted to \\ the Faculty of Graduate Studies and Research \\ in partial fulfilment of \\ the requirements for the degree of \\ Master of Computer Science \\ with \\ Specialization in Data Science
}

Department of Computer Science

Carleton University

Ottawa, Ontario, Canada

2020

Copyright (C)

2020 - Khadija Osman 
The undersigned recommend to

the Faculty of Graduate Studies and Research

acceptance of the Thesis

\title{
Studying the Health of the Bitcoin Ecosystem in GitHub
}

\author{
Submitted by Khadija Osman \\ in partial fulfilment of the requirements for the degree of \\ Master of Computer Science
}

Dr. Olga Baysal, Thesis Supervisor

Dr. Majid Komeili, Internal Examiner

Dr. Mohamed Al-Guindy, External Examiner

Dr. Matthew Holden, Chair of Defence

Dr. Michel Barbeau, Department Chair

Carleton University

2020 


\section{Abstract}

Bitcoin is a virtual and decentralized cryptocurrency that operates in a peer-to-peer network providing a private payment mechanism. It is a multi-billion dollar cryptocurrency, and hundreds of other cryptocurrencies are created based on it. Bitcoin is based on Open-Source (OSS) software development, and OSS is a convenient way to qualitatively measure software development and growth. This thesis presents the first comprehensive study of the Bitcoin ecosystem in GitHub organized around 481 most popular and actively developed Bitcoin related projects over eight years (2010-2018). Our work includes manual and data-driven categorization of the projects, defining software health metrics, classification of projects into three different classes of health, and evaluation of trends in the health of the ecosystem as a whole. Four classification algorithms such as decision tree, Support Vector Machines, K-Nearest Neighbor and Naïve Bayes are leveraged to predict the health of a project and compare the classifiers' performance.

The dataset used in this research is a combination of GHTorrent and a data collected during this study. The main findings suggest that the Bitcoin ecosystem in GitHub is represented by nine categories as result of manual categorization and 4 clusters based on the data-driven approach. Moreover, majority of the projects are assessed as "Low Risk". Our classification results show that decision trees outperform

other classifiers in predicting the health of a software projects with an accuracy of $98 \%$. 
I dedicate this thesis to my parents, without their love and support this would not have been possible. 


\section{Acknowledgments}

I, Khadija Osman, would like to express my sincere gratitude to my amazing supervisor, Professor Olga Baysal, for her continuous guidance, advice, and friendly discussions throughout my thesis. Our joint vision for this project, and her continuous efforts provided me valuable feedback and support, which made this work successful.

Besides my supervisor, I wish to express my deepest appreciation to all the thesis committee members for their valuable time, insightful comments and encouragement.

I would also like to pay my special regards to all my teachers who believed in me and helped me throughout the various aspects of this work, as their assistance was milestone in the completion of this project.

In addition, a wholehearted appreciation goes to my class and lab mates for their fruitful discussions, which were not only helpful but also encouraging. Last but not the least I am extremely grateful to my family, my parents, my husband, my siblings and my friends. They gave me a tremendous amount of support by motivating, encouraging and keeping me optimistic in difficult moments. 


\section{Table of Contents}

Abstract iii

\begin{tabular}{|l|l}
\hline Acknowledgments & v
\end{tabular}

Table of Contents $\quad$ vi

List of Tables $\quad \mathrm{x}$

List of Figures $\quad$ xi

Nomenclature xiii

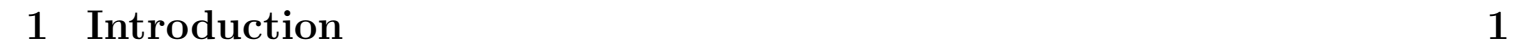

$1.1 \quad$ Bitcoin Software Ecosystem . . . . . . . . . . . . . . . . . . 1

1.2 Motivation . . . . . . . . . . . . . . . . . . . . . 3

1.3 Research Questions . . . . . . . . . . . . . . . . . . . . . 4

1.4 Contributions . . . . . . . . . . . . . . . . . . 6

1.5 Organization of the Thesis $\ldots \ldots \ldots \ldots \ldots$

\begin{tabular}{llr|}
\hline 2 & Background & 8
\end{tabular}

$2.1 \quad$ Cryptocurrency $\ldots \ldots \ldots \ldots \ldots \ldots$

2.2 Bitcoin Cryptocurrency $\ldots \ldots \ldots \ldots \ldots \ldots$. . . . . . . . . . . . 11

2.3 Software Ecosystem $\ldots \ldots \ldots \ldots \ldots \ldots$ 
2.3 .1 Software Ecosystem Health . . . . . . . . . . . . . . . . . 13

$2.3 .2 \quad$ Bitcoin Ecosystem . . . . . . . . . . . . . . . . . . . 14

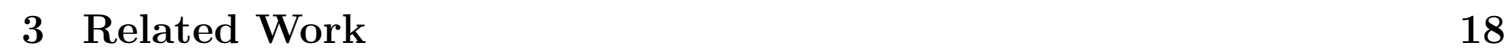

$3.1 \quad$ Software Ecosystem . . . . . . . . . . . . . . . . . . . . . . . 18

$3.1 .1 \quad$ Software Ecosystem's Architecture . . . . . . . . . . . . 18

3.1 .2 Identification and Evolution . . . . . . . . . . . . . . 20

$3.1 .3 \quad$ Software Ecosystem's Health $\ldots \ldots \ldots \ldots \ldots . \ldots \ldots$

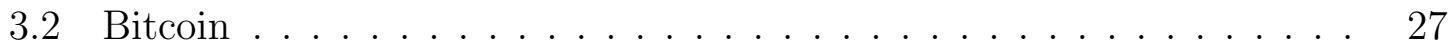

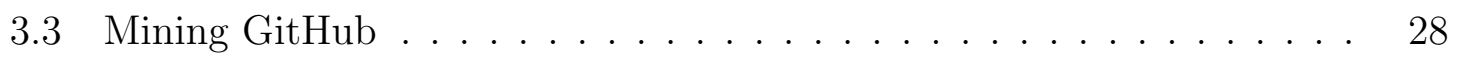

$3.3 .1 \quad$ Mining Bitcoin Data $\ldots \ldots \ldots \ldots$. . . . . . . . 30

3.3 .2 Software Ecosystems $\ldots \ldots \ldots \ldots$

\begin{tabular}{|ll|l}
4 & Methodology & 33
\end{tabular}

4.1 Data mining . . . . . . . . . . . . . . . . . . . . 33

4.1 .1 Project Selection and Preprocessing . . . . . . . . . . . . . 34

4.1 .2 Collecting Source Code Features $\ldots \ldots \ldots \ldots \ldots$

4.2 Defining the Bitcoin Ecosystem . . . . . . . . . . . . . . . 36

4.2 .1 Manual classification of GitHub projects . . . . . . . . . 36

4.2 .2 Clustering . . . . . . . . . . . . . . . . . . . . 40

$4.2 .2 .1 \quad$ Data Pre-processing . . . . . . . . . . . . . . . 40

$4.2 .2 .2 \quad$ Feature Selection . . . . . . . . . . . . . . . . . . . 41

$4.2 .2 .3 \quad$ K-means clustering $\ldots \ldots \ldots \ldots \ldots \ldots$

4.2 .3 Semi-supervised Clustering . . . . . . . . . . . . . . . . . 43

4.2 .4 Category Prediction . . . . . . . . . . . . . . . . 43

4.3 Health Analysis . . . . . . . . . . . . . . . . . . . . . . 44

4.3 .1 Health Metrics Definition and Heuristics . . . . . . . . . . 44

4.3 .2 Trends in Health of the Ecosystem as a Whole. . . . . . . . 50 
$4.4 \quad$ Predictive Modeling and Analysis $\ldots \ldots \ldots \ldots \ldots \ldots \ldots$

$4.4 .1 \quad$ Preparing Data for Training Models . . . . . . . . . . . . . 54

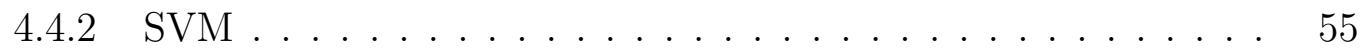

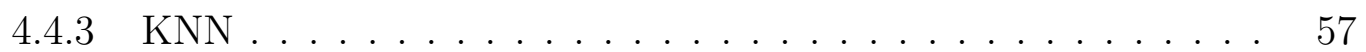

4.4 .4 Decision Trees . . . . . . . . . . . . . . . . . . 58

4.4 .5 Naive Bayes . . . . . . . . . . . . . . . . . . . . . 60

4.4 .6 Model Evaluation . . . . . . . . . . . . . . . . . . . 62

4.4 .7 Model explainability . . . . . . . . . . . . . . . . . 64

4.5 Tools and Libraries Used $\ldots \ldots \ldots \ldots$

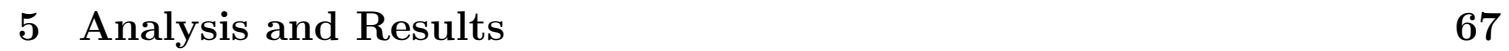

5.1 RQ1: What Categories Are Representing the Bitcoin Ecosystem in

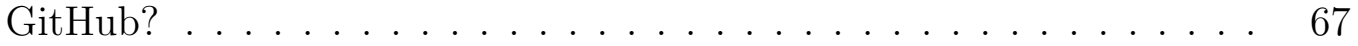

5.1 .1 Results of Manual Categorization . . . . . . . . . . . . 67

5.1 .2 Clustering Results . . . . . . . . . . . . . . . . . . 70

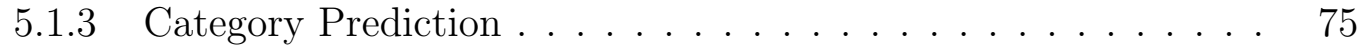

5.2 RQ2.1: How Healthy is the Bitcoin Ecosystem in GitHub? . . . . . . 77

5.3 RQ2.2: What Are the Trends in the Health of the Bitcoin Ecosystem? 78

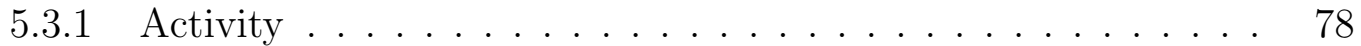

$5.3 .2 \quad$ Popularity $\ldots \ldots \ldots \ldots \ldots \ldots \ldots \ldots \ldots \ldots$

$5.3 .3 \quad$ Code Quality . . . . . . . . . . . . . . . . . . . . . 90

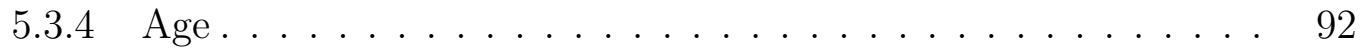

5.4 RQ3: How Accurately Can We Predict the Bitcoin Ecosystem's

Health? . . . . . . . . . . . . . . . . . . . . . . . . . . 9 92

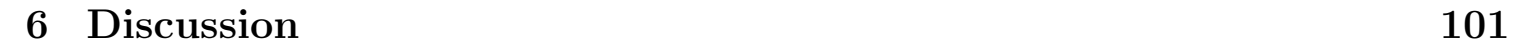

$6.1 \quad$ Main Findings $\ldots \ldots \ldots \ldots \ldots$

6.2 Implications for the Bitcoin Community in GitHub . . . . . . . . . 102 
$6.3 \quad$ Implications for the Bitcoin Community $\ldots \ldots \ldots \ldots \ldots \ldots$

6.4 Implications for Research Community . . . . . . . . . . . . . . 105

6.5 Threats to Validity $\ldots \ldots \ldots \ldots \ldots \ldots \ldots$

\begin{tabular}{|ll|}
\hline 7 Conclusions & 107
\end{tabular}

7.1 Summary of Contributions . . . . . . . . . . . . . . . . . 107

7.2 Future Work . . . . . . . . . . . . . . . . . . . . 108

\begin{tabular}{ll}
\hline List of References & 110
\end{tabular} 


\section{List of Tables}

$1 \quad$ Sofware ecosystem health metrics defined by Jansen et al. $|1|$. . . . . . 22

$2 \quad$ Features in the dataset: repository-level. . . . . . . . . . . . . . . . 37

$3 \quad$ Features in the dataset: commit-level. . . . . . . . . . . . . . . . . . 38

$4 \quad$ Keywords. . . . . . . . . . . . . . . . . . . . . . . . 39

5 Parameter optimization for decision trees. . . . . . . . . . . . . . . 43

$6 \quad$ Metric calculated . . . . . . . . . . . . . . . . . . 45

7 Metrics calculated. . . . . . . . . . . . . . . . . . 47

$8 \quad$ SVM models with different F1-scores. . . . . . . . . . . . . . . 57

$9 \quad$ Parameter optimization for decision trees. . . . . . . . . . . . . . . . 60

$10 \quad$ Confusion matrix $\ldots \ldots \ldots \ldots$. . . . . . . . . . . . . . 62

11 The categories in the Bitcoin software ecosystem. . . . . . . . . . . . 68

12 CART classification report. . . . . . . . . . . . . 76

13 Descriptive statistics for health categories. . . . . . . . . . . . . . . . 77

14 Number of projects created per year. . . . . . . . . . . . . 79

15 Descriptive statistics for projects' age. . . . . . . . . . . . . . . 92

16 SVM classification results. . . . . . . . . . . . . . . . . . . . . 93

17 CART classification report. . . . . . . . . . . . . . . . 95

18 KNN classification results. . . . . . . . . . . . . . . . 96

$19 \quad$ Naive Bayes classification results. . . . . . . . . . . . . . . . . 97 


\section{List of Figures}

1 Research roadmap. . . . . . . . . . . . . . . . . . . 5

$2 \quad$ Data collection process. . . . . . . . . . . . . . . . . 35

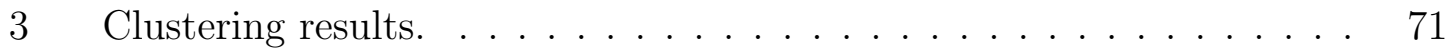

$4 \quad$ Cluster evaluation. . . . . . . . . . . . . . . . . . . . . 72

5 Semi-supervised clustering results. . . . . . . . . . . . . 75

$6 \quad$ Average number of commit per year per project. . . . . . . . . . . . 79

7 Change in the number of commits per year. . . . . . . . . . . 80

$8 \quad$ The average number of commits per category and per year. . . . . . . 80

$9 \quad$ Average number of commits per developer. . . . . . . . . . . . . . . . . 81

$10 \quad$ Average number of authors per year per project. . . . . . . . . . . . 82

11 Loss of contributors per year. . . . . . . . . . . . . . . 83

12 Developer retention rate over the years. . . . . . . . . . . . . . . . 84

13 Developer collaboration within the ecosystem. . . . . . . . . . . 85

14 Collaboration across different categories of the ecosystem. . . . . . . 86

15 Average number of issues per year per project. . . . . . . . . . . . 87

$16 \quad$ Average number of issues per year per category. . . . . . . . . . . . . 87

17 Average number of watchers per year per project. . . . . . . . . . . . 88

18 Average number of watchers per year and per category. . . . . . . . . . 89

19 Average number of forks per year. . . . . . . . . . . . . . 89

$20 \quad$ Average number of forks per category. . . . . . . . . . . . . . . . . 90 
$21 \quad$ Average code complexity per project. . . . . . . . . . . . . . . . . . 91

$22 \quad$ Average code complexity per category. . . . . . . . . . . . . . . . . . 91

23 Decision metrics for classifiers. . . . . . . . . . . . . . . . . . . . . 94

24 ROC curves. . . . . . . . . . . . . . . . . . . . . . . . . . . . 95

$25 \quad$ Feature permutation with increase in loss. . . . . . . . . . . . . . . 98

26 Weights after feature permutation. . . . . . . . . . . . . . . 99 


\section{Nomenclature}

\section{Abbreviations}

This thesis uses some common abbreviations existing in the domain of computer science. The following table lists the abbreviations and their meaning:

\begin{tabular}{cc}
\hline Abbreviation & Name \\
\hline AUC & Area Under Curve \\
BTC & Bitcoin Cryptocurrency \\
CC & Cyclomatic Complexity \\
CSV & Comma-Separated Values \\
DLT & Distributed Ledger Technology \\
GH & GitHub \\
KNN & K-Nearest Neighbor \\
LDA & Latent Dirichlet Allocation \\
OSS & Open-Source Software \\
OSSE & Open-source Software Ecosystem \\
ROC & Receiver Operator Curve \\
RQ & Research Question \\
SECO & Software Ecosystem \\
SVM & Support Vector Machine \\
TD-IDF & Term Frequency - Inverse Document Frequency \\
\hline
\end{tabular}




\section{Chapter 1}

\section{Introduction}

In this chapter, Section 1.1 introduces Bitcoin and software ecosystems, Section 1.2 discusses our motivation behind this work and Section 1.3 explains our research questions. In Section 1.4, we outline the contributions of this study. Section 1.5 defines the overall structure of the thesis.

\subsection{Bitcoin Software Ecosystem}

The invention of Bitcoin as a currency has introduced an information technology that facilitates secure and decentralized payment systems and tools for storage, verification, and auditing the information. Bitcoin is not a company that is run by an individual or a government, but it is a decentralized entity that is regulated by its users. Furthermore, it provides online transfers between two individuals based on cryptographic proofs rather than trust. Bitcoin relies on cryptographic algorithms and a distributed network of users, allowing them to mine, store and exchange their Bitcoins. Since the invention of Bitcoin in 2008, it has become the fastest growing

and most trusted cryptocurrency in the market. According to Williams [2], as of 2017 there are 2.8 to 5.8 million users of Bitcoin, and it is currently accepted by wellknown businesses as a payment solution. However, since Bitcoin is not controlled 
by a centralized authority it is manipulated by criminals to anonymously transfer funds $[3,4]$.

The concept of software ecosystem first appeared in a book by Messerschmitt and Szyperski in 2003 [5]. Since then, there are many different definitions to software ecosystems. Messerschmitt et al. [5] defined the software ecosystem as a collection of software products that have some given degree of cooperative relationships. On the other hand, Manikas et al. [6] tried to combine the technical, architectural and business viewpoints to single definition: "Software ecosystems are sets of software solutions functioning as a unit, enabling actors to automate activities and transactions." Precisely, they concluded in their study that a software ecosystem constitutes the interactions of a set of actors on top of a common technological platform that results in several software solutions or services. They added, that each actor in the ecosystem is motivated by a set of interests and business models and connected to the rest of the actors and the ecosystem as a whole with cooperative relationships, while, the technological platform is structured in a way that allows the involvement and contribution of the different actors. In this work, we adopt the definition of a software ecosystem as proposed by Jansen et al.: "software ecosystem a set of projects, and actors functioning around a common technological platform or a market; they exchange knowledge, resource, and artifacts" [7]. Thus, our definition is also concerned about projects and people involved in developing them for the purpose of sharing their knowledge, expertise, and contributions.

In this research, our goal is to define the Bitcoin software ecosystem in GitHub in terms of its available projects and its contributors. Furthermore, we will perform an analysis of the health of the defined ecosystem. 


\subsection{Motivation}

Currently, the total supply of Bitcoins available is 21 million, and the reward for Bitcoin mining is 6.25 Bitcoins. However, the reward for Bitcoin mining is cut in half every four years leading to a regular decrease in the incentive to mine Bitcoin, and with it a decrease in the incentive to develop software around Bitcoin as the reward to do so diminishes. Furthermore, another concern that miners face is related to how they would be paid if the supply of Bitcoin overruns. One possible solution would be charging a transaction fee. Nevertheless, Bitcoin remains the leading cryptocurrency in the market, so it is important to explore its software ecosystem and investigate whether it has formed a healthy software ecosystem.

The Bitcoin ecosystem is composed of five main players. First one is miners - a group of people who create Bitcoins by using a huge number of resources and time. Miners spend their Bitcoin in exchange for fiat or other goods. The estimated number of coins mined per month is $108 \mathrm{~K}$ with an operational cost of US\$18 million [8]. Miners reinvest $50 \%$ of their income, exchange $25 \%$ and hoard the rest of the $25 \%$. The second category in this chain is traders. Traders provide a platform for the rest of the entities to sell and buy Bitcoins for the purpose of profit. The third category is merchants, the businesses that accept Bitcoins as payments. Fourth, hoarders are people who hold on to their Bitcoins for a time when they would have value in millions. The last category is consumers, who spend Bitcoins for goods and services. Besides these five groups, there are many external factors that affect this ecosystem. For example, the

economy and cost of electricity. Each of these categories are explained in more detail in Section 2.3.2.

The motivation behind studying this ecosystem in GitHub is that GitHub is the most favored and popular OSS development platform. GitHub is hosting more than 10 million software repositories (i.e., repos) and over millions of developers collaborate 
with each other and have shared repos in GitHub. Besides, Bitcoin's main repo is hosted on GitHub. Furthermore, this study can provide useful insights to the developers and researchers in the field of OSS development, cryptocurrency, and software ecosystem.

\subsection{Research Questions}

The objective of this study is three-fold: 1) we are interested in defining the structure of the Bitcoin ecosystem in GitHub by categorizing the Bitcoin related GitHub projects, 2) we aim to evaluate the health of the defined Bitcoin ecosystem, and 3) we want to apply predictive modeling to determine how accurately we can predict the health of an individual project within the ecosystem and to analyze the trends in the health of the ecosystem as a whole. Figure 1 shows the overall research roadmap that connects our research questions to the process we followed.

In this thesis, we aim at answering the following research questions:

1. RQ1: How the Bitcoin ecosystem is represented in GitHub? Bitcoin is the leading cryptocurrency, understating its development in GitHub can help us define its open-source software ecosystem (OSSE). Furthermore, we can understand the ecosystem in more detail by categorizing the projects into different types of applications of Bitcoin. Such classification can enable the researchers in the field of cryptocurrency to better understand the public and open source side of the Bitcoin ecosystem, and they can conduct further studies that are related to OSS practices and processes.

\section{RQ2.1: How healthy is the Bitcoin ecosystem in GitHub?}

Software is central to the Bitcoin function, and it follows OSS development. 


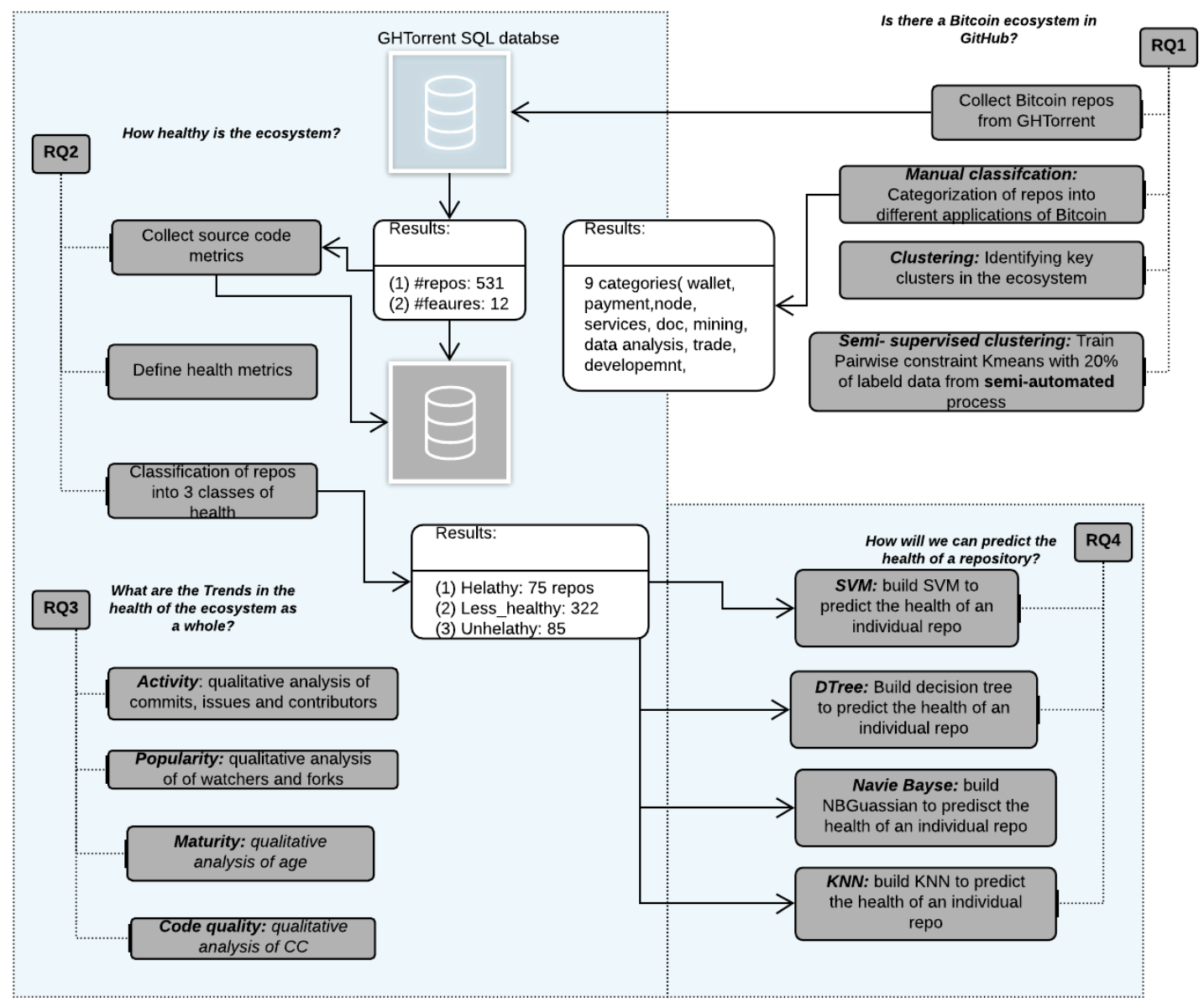

Figure 1: Research roadmap.

Maintaining a healthy ecosystem of OSS is also a prerequisite for the sustainable development of open-source communities. Thus, studying the health of the Bitcoin ecosystem can provide practical insights into the well being of the projects in the ecosystem and the ecosystem as a whole. Based on the results we can provide some potentially useful insights to the Bitcoin community to help them adapt the practices essential for maintaining a healthy OSSE.

\section{RQ2.2: What are the trends in the health of the Bitcoin ecosystem?}

By answering this question we can understand the changing trends in the popularity, activeness, and code quality of the ecosystem. Such findings can provide 
us with the indication of whether the health of the ecosystem is improving overtime or deteriorating. As an outcome, we can provide some potential guidelines and recommendations to the Bitcoin development community in the GitHub on maintaining the health of their projects that comprise the Bitcoin ecosystem.

4. RQ4: How accurately can we predict the health of the Bitcoin ecosystem?

Open source developers often face problems when choosing a project to contribute to. There are many different approaches to suggest and recommend projects to newcomers. The method proposed in this study is an efficient and data-driven approach to label GitHub projects into different classes of health. The classification of the project is based on the social and technical features of OSS. We aim to build classification models and predict the health of a project based on the social and technical features that we extract from GitHub. The results to this question can help developers to choose a project which is mature, popular, actively developed, and has a high quality of the source codebase.

\subsection{Contributions}

This thesis makes the following contributions:

1. Mapping out the Bitcoin ecosystem in GitHub by collecting and analyzing all relevant projects.

2. Classification of the Bitcoin projects in GitHub into different categories: datadriven vs. manual approach.

3. Extraction and collection of projects' features to define the health metrics based on both social and technical aspects of software projects. 
4. Classification of the projects in the Bitcoin ecosystem into three classes of health.

5. Development of four classification algorithms to predict the health of a project in the Bitcoin ecosystem.

6. Analysis of the trends in the ecosystem's health based on the four metrics such as popularity, maturity, activity, and code quality.

7. A set of recommendations to researchers and developers in the field of cryptocurrency, software ecosystem and GitHub.

\subsection{Organization of the Thesis}

The rest of the paper is organized as follows. Chapter 2 presents the background on software ecosystems in general and the Bitcoin ecosystem, while Chapter 3 discusses the related work. In Chapter 4, we explain the dataset and methodology we used in this research. Chapter 5 presents the results of our analysis. Chapter 6 discusses our findings, some challenges and implications. Finally, Chapter 7 concludes our work. 


\section{Chapter 2}

\section{Background}

In this chapter we will discuss cryptocurrency (Section 2.1), Bitcoin (Section 2.2), software ecosystems (Section 2.3), health of a software ecosystem (Section 2.3.1), and the Bitcoin ecosystem (Section 2.3.2.

\subsection{Cryptocurrency}

Cryptocurrency is a virtual or digital currency used as a medium of exchange in online transactions. It uses cryptography for the purpose of secure transactions and to control the creation and maintenance of new units of a particular cryptocurrency. It is decentralized, there is no one authority who controls the system; rather a group of miners, or owners of the coins control the transactions of the currency. It uses the blockchain technology to achieve decentralization, immutability and transparency [9].

Cryptography: Cryptography is the process of securing the communication and information. It uses mathematics rules to change the information in a way that no third party can interpret it. Cryptography is used in communication to insure the following:

1. Confidentiality: Insures that the information is not understood by any other person than the intended receiver. 
2. Integrity: It insures that the information is not changed between the sender and the receiver.

3. Authentication: It enables that the receiver and the sender can identify and authenticate each other.

4. Non-repudiation: It is to make sure that the sender and receiver can not deny at the later stage of the information exchanged.

This is achieved with the help of cryptographic algorithms. These algorithms are used to encrypt and decrypt the message being communicated. They do this by generating a pair of public and private key, digital signing and verification, and key exchange. There are two kinds of cryptography algorithms, single-key symmetric algorithms where both the sender and receiver use the same key for encryption and decryption. The other type is public-key asymmetric algorithms. They use two keys, a private and a public one. Private key is used to encrypt the information and not being shared with the receiver. The receiver uses the public key which is associated with the sender to decrypt the message. There are some standard public-key algorithms such as Rivest-Shamir-Adleman (RCA) used over Internet and Elliptic Curve Digital Signature Algorithm (ECDSA) used by Bitcoin.

Blockchain: Blockchain, also known as Distributed Ledger Technology (DLT), makes any digital asset immutable and transferable through decentralization and cartographic hashes. It is a growing list of blocks, where each block has a cryptographic hash of the previous block, a timestamp, and transaction data. The blockchain is managed by a peer-to-peer network. Furthermore, it is an open and distributed on all the nodes in the network. Blockchain has three main concepts: 1) Blocks, 2) Miners, and 3) Nodes.

Blocks: Every chain has multiple blocks and each block has the following elements: 
1. The data in the block, in case of cryptocurrencies, is the transaction.

2. A 32-bit whole number called a nonce. The nonce is randomly generated when a block is created, which then generates a block header hash.

3. The hash is a 256-bit number added to the nonce.

When the first block of a chain is created, a nonce generates the cryptographic hash. The data in the block is considered signed and forever tied to the nonce and hash, unless it is mined.

Miners: Miners create new blocks on the chain. In a block chain every block has its own unique nonce and hash, and references the hash of the previous block in the chain, so mining a block is not easy, especially on large chains. Miners use special software to solve very complex math problem of finding a nonce that generates an accepted hash. There are roughly four billion possible nonce-hash combinations that must be mined before the right one is found, because the nonce is only 32 bits and the hash is 256 bit long. When that happens miners are said to have found the "golden nonce" and their block is added to the chain. Making a change to any block earlier in the chain requires re-mining not just the block with the change, but all of the blocks that come after. This is the reason that it is extremely difficult to manipulate the blockchain technology, since finding golden nonces requires an enormous amount of time and computing power. When a block is successfully mined, the change is accepted by all of the nodes on the network, and the miner is rewarded financially.

Nodes: One of the main concepts in the blockchain is decentralization, and this is the reason that no single computer or organization can own the chain. Thus, it is a distributed ledger via the nodes. Nodes can be any kind of electronic device that maintains copies of the blockchain and keeps the network functioning. Every node has its own copy of the blockchain and the network must algorithmically approve any newly mined block for the chain to be updated, trusted and verified. Since blockchains 
are transparent, every action in the ledger can be easily checked and viewed. Each participant is given a unique alphanumeric identification number that shows their transactions. Combining public information with a system of checks-and-balances helps the blockchain maintain integrity and creates trust among users.

\subsection{Bitcoin Cryptocurrency}

Bitcoin cryptocurrency (BTC) is the first digital currency created in the year 2008 and is based on OSS. The first paper on Bitcoin was published by Satoshi Nakamoto [10], but the original creators of this cryptocurrency remain unknown. Some researchers believe that the cryptocurrency was created by Satoshi, while others think it was a group of people called Satoshi. At the time of writing, according to CoinMarketCap [11], Bitcoin is the leading cryptocurrencies in the market. BTC allows peerto-peer transactions over the Internet, which are recorded in a public ledger. The ledger is simply a network of peers. Every peer has a record of the complete history of all transactions and the balance of every account. A transaction is a file that says: "Bob gives X Bitcoins to Alice" and is signed by Bob's private key. This process is the basic public key cryptography. After signed, a transaction is broadcasted in the network, sent from one peer to every other peer.

Bitcoin transactions are documented in a virtual ledger called "the blockchain". The ledger follows a decentralized structure. This ledger is accessible to everyone in the Bitcoin network, everyone can view the transactions. The architecture of Bitcoin gives complete control over one's account unlike the bank systems, there is no centralized authority controlling Bitcoin. Anyone having access to the Internet can generate Bitcoins through a process called "mining".

Mining: Mining Bitcoins follows the process discussed in Section 2.1. When users allocate computer processing power towards the mining of Bitcoins, they are 
rewarded with transaction fees and newly created coins. Through the mining process, we can update the ledger of Bitcoin (blockchain) which is done with high powered computers known as ASICS. The miners race against each other to guess a random number first, by guessing correctly the miner is awarded 12.5 Bitcoins. At the time of writing, 12.5 is the current rate of Bitcoin, meaning that adding one block to the Bitcoin ledger values 12.5 Bitcoins. The random number (nonce) is used to solve a mathematical question generated by the system.

Cryptographic algorithm: Bitcoin use ECDSA to make sure that the coins are used by the right person. It has three main concepts: (1) Private key: This is a 256-bit random integer generated by the miner. This is only known to the miner and is associated with him/her on the blockchain. (2) Public key: This key is associated and calculated with a private key and is not supposed to be a secrete. This key is used when sending Bitcoin to another party. (3) Signature: It is a number that proves that a transaction took place on the blockchain. It is mathematically generated from a hash of something to be signed plus the private key. Wallet applications, discussed in Section 4.2.1, are used to store private key and keep track of public keys.

At the time of writing, Bitcoin is accepted by many legal merchants and charities [12]. Bitcoin gains more popularity throughout the years and it is accepted as a payment method by the most recognized organizations like Microsoft 13 and Amazon [14. Amazon is not accepting Bitcoin as a payment method directly, but you can buy things in Amazon through a third party application purse.io [15. According to the CoinMarketCap [11] Bitcoin has taken over the market of cryptocurrencies, because the most popular organizations such as Wikipedia [16, Microsoft [13], Expedia [17], and Overstock [18] accept it as the payment method. Wikipedia [16], the world's largest open-source encyclopedia, accepts donations with Bitcoins through bitpay [19. Microsoft [13 allows users to top their Microsoft account with Bitcoins through their Bitcoin wallet. Expedia, a travel company, accepts Bitcoin as payment 
though coinbase 20].

\subsection{Software Ecosystem}

Unfortunately, in contrast to natural ecosystems, there is no common definition for Software Ecosystems (SECO). A SECO can be defined and interpreted in different ways, for example, it can be business-centric or platform-centric [21]. Some researchers define an software ecosystem as a network of entities or stakeholders that are gathered and interacting around a single platform 22 24]. Similarly, a SECO is defined as a collection of software projects that are developed together and co-evolve, due to project relevance and shared developer communities, in the same environment 25,26.

\subsubsection{Software Ecosystem Health}

As the software development is growing fast, there should be some measures to keep the development environment healthy. Thus, the right precautions should be taken towards a healthy SECO. Furthermore, there should be a set of reliable maintenance measures to keep a supportive and collaborative environment. From a biological perspective, health means that an organism's vital system is working normally at a given

time [27]. According to [27], this definition can be extended to SECO software ecosystem health as well. For a SECO to be healthy, all the contributing entities should perform well. Whether its the quality of the software, or a healthy communication among the developers of a certain project.

Health problems can be very diverse in nature and have many different causes. A healthy communication among the contributors of an ecosystem plays an important role. For example, communication styles of open-source communities, such as the one of the Linux Kernel community, caused a prominent developer to quit [28]. The 
blog 28 also explains that how the community suffered due to the unexpected leave of a central contributor to the bug handling community of Gentoo Linux. Another example of health issues is faced by npm ecosystem due to an unexpected wait of a key member to publish his package in 2016 [29]. Many other packages directly or indirectly depended on the this package. The delay was caused due a dispute regarding the package name. Besides, Marsan et al. 30 identified some social and technical problems that are being faced by the community of open-source SECOs.

\subsubsection{Bitcoin Ecosystem}

The Bitcoin ecosystem brings multiple parties to a common purpose. Cusumano 31 discussed Bitcoin as an ecosystem for the first time. According to him, wallet services, exchange markets, and business that accept Bitcoin as a payment solution compose Bitcoin ecosystem [22], where different kinds of agents interact, trade, and communicate through digital channels. The Bitcoin ecosystem can be defined from different perspectives, for example, we can define it as consisting of three parties such as developers, miners, and investors. If we want to define it from a business point of view, we can define it as miners, traders, merchants, hoarders, and consumers having different roles in maintaining this cryptocurrency. These parties along with the services they provide can be considered as Bitcoin ecosystem.

The Spark Capital [32] has explained the Bitcoin ecosystem in detail. They divided the ecosystem into the following seven entities, interacting to maintain the ecosystem:

1. Wallets: A Bitcoin wallet is a software program where Bitcoins are stored, it is also used to send and receive coins. More specifically wallets are used to store public and private keys for a node in the Bitcoin network. The sender uses the public key of the recipient to send or receive coins. Majority of the cryptocurrencies have their own wallets that work only for that cryptocurrency. 
There are many kinds of wallets you can use including online, offline, mobile, hardware, desktop, and paper. A wallet can be either a full-Bitcoin node, which stores the complete network locally or a lightweight client. The full node wallets need heavy resources to store the whole blockchain ledger, but is more secure than lightweight-Bitcoin clients. While a lightweight-Bitcoin client does not store the block chain history, it uses remote server. It is fast and efficient but less secure. Some examples of popular Bitcoin wallets are Bitcoin Core wallet [33], Samourai [34], Armory [35], and GreenAddress [36].

2. Payment processors: These entities enable users to accept and make payments in Bitcoins. There are two types of payment processors, payment services providers and payment networks. The first type provides services for Bitcoin exchanges and offline/online merchants. The second type are independent networks with Bitcoin as one of the services they provide. Both entities generate revenue by charging fees for transferring funds, security, and subscription. Ripple Labs [37] is an example of payment networks. It is a distributed payment network with Bitcoin as one of the currencies. There are many payment service providers such as BitInstant [38, bitpay [19], BIPS (2011-2013) [39], coinbase [20], etc.

3. Exchanges/Trades: These entities allow users to trade Bitcoins for cash or stock shares. There are three types of platforms providing exchange services: (1) OTC Bitcoin Exchanges: They aggregate the Bitcoin value and display the outstanding Bitcoin supply and demand. They also allow users to transact directly with each other. There are many examples of this service, e.g., LocalBitcoins [40]. (2) Spot and forward exchanges: They allow users to transact using a centralized system. It also exchanges Bitcoin for multiple currencies, and bets on the future price of Bitcoin. BitStamp [41] is a European based 
Bitcoin exchange and is one of the popular exchanges in the market. (3) BTC Stocks: They allow users to invest Bitcoins in exchange for shares in a business. All the three types of services generate revenue by charging users deposit and withdrawal fees.

4. Borrowing and Lending: These entities allow users to borrow and lend Bitcoins and earn interest. There are two types of services in this category: (1) Peer-to-Peer: In this category they let the borrower to choose the lender according to their investment criteria. Borrowers are also graded according to creditworthiness just like the traditional loan system. (2) Bank-like Lending: In this method the borrower and lender are not identified to each other. They both interact with a central authority to borrow or lend Bitcoins. Bitbond [42] is an example of the first type, it connects borrowers and lenders directly. BitLender [43] is a website, providing loans, credit checks and certificates of deposits.

5. Hardware and Equipment: This group of entities provide hardware and equipment to the whole Bitcoin infrastructure. There are two types of hardware: (1) Mining Hardware: It includes hardware which provides a secure method to store the Bitcoin ledger and also considers the computations power and electricity consumption. Butterfly labs [44], Avalon, and HashFast Technologies are some of the high speed mining and encryption processors for Bitcoin mining. (2) Bitcoin ATMs: They are created specifically for cashing Bitcoin into local currency. It also supports depositing Bitcoins into an individual account. Lamassu Bitcoin venture [45] is a one way Bitcoin ATM, just for cashing Bitcoins into local currency. While, Robocoin 46 supports both buying ans selling Bitcoins.

6. Investment Vehicles: These entities provide services to invest in Bitcoins for the purpose of generating return. They are many services who provide these 
services including Bitcoin ETFs. It tracks Bitcoin price and functions like a traditional stock exchange.

7. Others: Beside the entities explained above, there are many other concepts in Bitcoin ecosystem. We provide a brief definition for some of them next. (1) Miner lease provides Bitcoin mining contracts for investment. For example, Bitcoin cloud mining [47] allows users to earn coins without mining hardware, Bitcoin mining software, electricity, bandwidth or other offline issues. (2) SatoshiDice [48] is a leading Bitcoin gaming site. (3) Coinstar 49] is a platform through which you can donate your coins to charity. (4) Bitcoinget [50] allows users to earn Bitcoins for watching a video and completing a simple task. (5) Cryptocard [51] is an international prepaid Bitcoin MasterCard to get money to the people who do not accept Bitcoins.

To study Bitcoin software ecosystem in GitHub and its health, we consider all the repositories/projects in GitHub relevant to these entities. These include Bitcoin core projects, wallet apps (mobile, desktop), exchange services applications, and platforms that provide libraries to develop different kind of apps. 


\section{Chapter 3}

\section{Related Work}

In this chapter, we present state-of-the art research related to the definition of software ecosystem and ecosystem's health (Section 3.1), Bitcoin cryptocurrency (Section 3.2), and mining GitHub data (Section 3.3).

\subsection{Software Ecosystem}

There is an extensive research in the field of software ecosystems (SEs) in the past 10 years. Manikas et al. [52] found that there is a significant increase in the published papers in this field each year form 2007 to 2014. Some researchers consider such rapid evolution of the large ecosystems as one of the reasons, beside the product-line, and global development, for a growing complexity in software development [53]. The research state-of-the-art in this field is organized around three main concepts such as defining the architecture of SEs, identifying new SEs, evolution and health of SEs. We will discuss the research related to each concept in the following sections.

\subsubsection{Software Ecosystem's Architecture}

There is a vast number of studies towards defining the architecture of a software

ecosystem. Some think that it should be construed around project relevance [25], 
while others say that it should be business oriented [7,54,55]. For example, Lungu et al. [56] state that the structure of an ecosystem can be well defined by the project relevance in an ecosystem. Some researchers have conducted user studies with experts in this domain to develop a definition of a software ecosystem. For example, Serebrenik et at. [57] have conducted a study with 26 software ecosystem experts. The respondents answered the question related to how they define as an ecosystem and provided their justification on such definitions. The authors have also identified the current challenges and future trends in the field of software ecosystems research.

Similarly, Franco et al. 58 analyzed 82 papers to understand open-source software ecosystems. The purpose of their study was to evaluate the current state of the art in OSS ecosystems (OS-SECOs) research, specifically: (a) what are the most relevant definitions related to OSSECOs; (b) what the particularities of this type of SECO are; and (c) how the knowledge about OSSECO is represented. Furthermore, Manikas et al. 52 have conducted an extensive literature review of software ecosystems form 2007 till 2014. They analyzed a total of 231 papers and identified a set of challenges in this field. A similar study by Kondel et al. [59] has defined an ecosystem in the terms of the following building blocks:

1. Actors,

2. Actors incentives,

3. Contribution and environment, and

4. Common technology: ecosystems emerge around a shared technology. It can be of two kinds: 1) at engineering time (e.g., infrastructure, IDEs, SDKs, APIs, or standards) or 2) at execution time while the ecosystem is in operation (RTEs, platforms, frameworks, or protocols).

Hyrynsalmi et al. [60] have summarized the definitions in the literature into three 
groups. The first group sees the software ecosystems (SE) as a special case of business ecosystem, similarly to Jansen et al.'s view [7]. According to Jansen et al. [7], “ $a$ software ecosystem is a set of businesses functioning as a unit and interacting with a shared market for software and services, together with the relationships among them. These relationships are frequently underpinned by a common technological platform or market and operate through the exchange of information, resources and artefacts.". The second group includes the SEs around open-source projects, its dependencies and community. Lungu et al. 25] define open-source SE as a "collection of software projects which are developed and co-evolve in the same environment". The last group defines SE as a set of developers or organizations working together on a set of development projects.

Our research focuses on exploring how a well-known Bitcoin ecosystem is represented and defined in GitHub. Following the second group of SE, we define the structure of the Bitcoin ecosystem in GitHub in terms of the Bitcoin repositories, the developer community, and a shared framework.

\subsubsection{Identification and Evolution}

In this section, we highlight the literature on the evolution of existing SEs and identification of new ecosystems. The majority of these studies explores different aspects of well-known ecosystems. The well-known ecosystem literature includes but not limited to programming languages, mobile software, and different development platforms. In programming languages, Python and $\mathrm{R}$ are well studied ecosystems by the researchers. For example, Granger et al. 61] have studied Python as an ecosystem for scientific studies, while Ma et al. [62] have identified the dominant projects in the Python ecosystem. Beside Python, R language and its CRAN have been studied by

many researchers in different contexts. For instance, Claes et al. 63 have studied 
how R packages are being maintained. Furthermore, Adams et al. [64 have studied the evolutionary characteristics of R software ecosystem. Similarly, Vasilescu et al. 65] have investigated how social question and answer sites are changing knowledge focused on $R$ package. Beside the programming languages, platforms such as .NET and Smalltalk have been identified and studied as an ecosystem. Robbes et al. 66 have focused and studied Pharo development platform for the Smalltalk programming language, and Microsoft's.NET platform (supporting a variety of programming

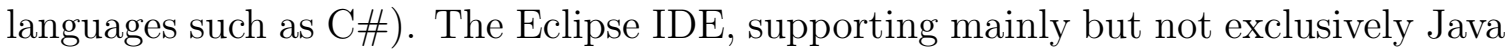
programming, and allowing third parties to contribute with different plugins have been mentioned 10 times by survey respondents. This software ecosystem, supported by IBM, has been extensively studied [67 70]. Mobile ecosystems are also extensively studied by the researchers in this field, particularly in the domain of open-source software research. For example, Harman et al. [71] performed data mining and analysis of app stores for Android and Apple. Similarly, Hyrynsalmi et al. [72] have studied and analyzed the app store for multi-homing systems. Furthermore, Bettenburg et al. 73] have studied contribution management in two different open-source software ecosystems. They have considered both for-profit (ANDROID) and non-profit (Linux kernel) ecosystems. They have found that both ecosystems have different contribution management styles and adopt different approaches. Some researchers think that software ecosystems are not self contained, but there are ecosystems which share certain packages. For instance, Constantinou et al. [74 have investigated cross-ecosystem packages in 12 known ecosystems. Similarly, Teixeira et al. 75] have claimed that software ecosystems do not evolve in isolation but with other SEs. They have studied Open Stack SE in attempt to understand how it evolves with other SEs.

Beside the aforementioned domains where SE are studied extensively, crytocurrencies have been also studied as SEs $22,24,76,77]$. We discuss the studies related to cryptocurrencies in Section 3.2. Our study aims at identifying and studying different 
aspects of the Bitcoin ecosystem within an open-source platform, i.e., GitHub. In the following section we discuss the research on the health of SEs.

\subsubsection{Software Ecosystem's Health}

The health of a SE defined by Jansen et al. [1] refers to the normal functioning of the projects that constitute the ecosystem and of the ecosystem as a whole. Jansen et al. 1] have provided a comprehensive overview of the health metrics shown in Table 1 that can be used to determine the health of an open-source ecosystem. They created a framework by creating an inventory of all the health indicators. The framework they created is based on three pillars: (1) Productivity, (2) Robustness, and (3) Niche creation. Each pillar is further divided into three levels: (1) Theory, (2) Network, and (3) Project level. Metrics that particularly address growth, such as new related projects, are put into the productivity category. Metrics that address robustness, such as the number of active projects, are defined in the robustness category. Furthermore, they evaluated the framework with four projects.

Table 1: Sofware ecosystem health metrics defined by Jansen et al. [1].

\begin{tabular}{l|l|l}
\hline Metric (label) [health category] & Source & Data Gathered On \\
\hline New projects (Met01) [productivity] & GitHub & January 4, 2018 \\
Commits for projects (Met02) [productivity] & GitHub & January 4, 2018 \\
Number of forks (Met03) [productivity] & GitHub & December 28, 2017 \\
Number of active projects (Met04) [robustness] & GitHub & December 28, 2017 \\
Interest(Met05) [robustness] & Google Trends & January 4, 2018 \\
Potential for niche creation (Met06) [niche creation] & Company website & January 2, 2018 \\
Actual niche creation (Met07) [niche creation] & GitHub & December 28, 2017 \\
\hline
\end{tabular}

A group of researchers [27] have initiated an interdisciplinary project (SECOHealth) to contribute to the software engineering community by recommendation tools for improving the health of a software ecosystem. This project is a collaboration between the research teams of Polytechnique Montreal (Canada), the University 
of Mons (Belgium) and Laval University (Canada). The project is considered as a successor for their ECOS (ecological studies of open source software ecosystems) project [78]. As part of this project, the researchers have conducted many studies between 2012 and 2017 63,64,79,84. Their aim is to equip the key actors of an ecosystem with the right tools to maintain the ecosystem health. For their methodology, they adopted a socio-technical perspective, since the social and technical layer of a software ecosystem is strongly correlated [85]. Following this perspective, they considered three levels of health: social, technical and phenomenological. Technical characteristics include traditional software quality metrics such as error rate, growth rate and so on. Social characteristics include characteristic of contributors such as turnover rate and interaction among the community. Phenomenological health characteristics include the amount of company involvement such as paid contributors and market share and so on. Furthermore, they conducted face-to-face interviews with open source summit for the Linux foundation in Prague 2017 to identify health problems faced by the experts. Eventually, they summarized their goals of this project into the following points which are based on both social and technical perspectives:

1. Define a conceptual model of SECO health; explore analogies from other scientific disciplines such as ecology and toxicology;

2. Determine indicators capable of measuring the different aspects of SECO health;

3. Determine events that affect the health of a SECO and its constituent projects;

4. Empirically validate these health indicators and events, both qualitatively and quantitatively;

5. Build and evaluate models to predict the impact of a given event on SECO health;

6. Build and evaluate a socio-technical dependency model to understand how health problems propagate throughout a SECO; and

7. Propose a catalog of guidelines and recommendations for supporting SECO 
health.

The health problems propagate to the ecosystem due to the social and technical dependencies between the projects [86]. Social and technical problems are dependent events, usually linked through cause-effect relations [30]. They used an original approach consisting of interviews with experts, content analysis, and root-cause analysis (RCA). They interviewed the experts in open-source software ecosystem community to find out the health problems they believe their ecosystems face. Three of the authors of this article interviewed 10 OSSECO experts during the 2017 Open Source Summit (OSSummit) in Prague. They identified a number of technical and social health problems. The following are the problems mentioned by at least $50 \%$ of experts:

The important social problems are (in order of importance):

1. Loss of contributors,

2. Lack of interactions between contributors,

3. Low number of contributors, and

4. Low number of contributors.

Two important technical problems are:

1. Poor code quality, and

2. Low number of code commits.

They concluded their study by identifying that loss of contributors and poor code quality are important problems that effect the health of an ecosystem.

Some researchers study the health of SEs from different aspects. For example, Dijkers et al. [87] have explored whether health of a software ecosystem effects the financial position of an open-source company. They performed a case study on two open-source companies Cloudera and Hortonworks. They used the metrics defined by [1] to evaluate the health of two organizations. As a result, they found that Cloudera is healthier in terms of robustness and niche creation, while Hortonworks is 
healthier in terms of productivity. Financially, Cloudera performs better than Hortonworks. They have built a hypothesis that the health of software ecosystem has a positive relation with financial position of a company. Niche creation health is the main contributor to this relationship, while robustness is a minor indicator of this relationship, while software ecosystem productivity health has little to no influence. They measured the financial performance with metrics such as share price, return in equity, earnings per share, total revenue, and Altman's Z-score. They also performed an observational study, which is "a study of a real-world case without performing an intervention" [88]. The selection of companies was based on the following criteria: 1) the company should be open-source, and 2) they should have gone public, so its financial information is available. The data was gathered in year 2017, from companies' GitHub pages, using a combination of scarping tools and GHTorrent dataset [89]. For the analysis of data, developments in SECO health and financial performance were judged on desirability. Desirability is defined as improvement in the metrics. That is, if the measure for a certain metric has improved over time, then the health for that metric is changing in a desirable way. The following categories were used: "+" to indicate a positive desirability, "-" to indicate a negative desirability, and "0" to indicate stagnation.

A similar study was conducted by Alami et al. [90]. They have explored the health of three most successful e-commerce platforms according to their shares in the market. They used the metrics defines by Jansen et al. [1]. They aim to answer two questions, including: 1) what makes these platforms healthy, and 2) how it complements the knowledge on SEs health. They evaluated Magento, WooCommerce and PrestaShop, which holds $28.3 \%, 17 \%$ and $7.5 \%$ shares of e-commerce, respectively. Magento is an eBay Inc. company, an open-source content management system for e-commerce web sites that was launched in 2008. PrestaShop is another open source e-commerce 
solution that was launched in August 2007. WooCommerce is the most popular ecommerce plugin for Wordpress, and it was launched in late 2011. Furthermore, Soussi et al. [91] have explored the Vuforia ecosystem's health by mining the Vuforia platform's application repository. Vuforia is a platform for Augmented Reality (AR) that provides Application Programming Interfaces (API) in $\mathrm{C}++$, Java, Objective-C, and the .Net languages through an extension to the Unity game engine. The source of their dataset is the developer website and forums.

Some researchers have investigated the challenges related to quality assurance of a software ecosystems which is critical in embedded systems [92]. They performed a systematic mapping of literature review on the health of SEs based on six studies. Beside this study, there is a number of literature review studies published in the last few years regarding ecosystem health in the context of software. For example, Hyrynsalmi et al. [93], Manikas et al. [94], and da Silva Amorim et al. [95] have addressed the state-of-the-art of software ecosystem health research. Silva et al. 93 analysed 23 research papers and Hyrynsalmi et al. [93] reviewed 38 research papers. Hyrynsalmi et al. [60] have also provided critics on the current health and evaluation metrics in the literature. They adopted a business oriented definition of a SE [54, 55]. They also provided three proposals that could help researchers in the field of SE health. They criticized four points regarding health metrics: 1) majority of the metrics are based on a static view of an ecosystem, while the ecosystem is constantly changing, 2) the studies do not explain whether the metrics have proactive power to predict or only reactive power to explain past events, and 3) the studies do not define the question "healthy for whom?" as it will add more complexity to the ecosystem health concept. Finally, they proposed the following. The first proposal of this study is that each research should answer the following three questions 1) What type of characteristic is measured from an ecosystem? 2) How do the measured characteristics impact the ecosystem? 3) Why is it measured? [60]. The second proposal is to focus on 
a certain type of ecosystems e.g., open-source. The third proposal considers the level of analysis. They argue that, when measuring a system, we should use system-level metrics and characteristics, instead of individual project-level measures.

\subsection{Bitcoin}

Since the creation of Bitcoin in year 2009, the cryptocurrency has become a trending topic in a diverse field if research, from security to the blockchain technology. We will discuss some studies that explore Bitcoin within the open-source community. For example, Baura er al. [96] have explore whether Bitcoin is an asset or a medium of exchange. Furthermore, they investigated the current uses of Bitcoin and its potential uses in future. Bitcoin is among the top cryptocurrencies according to Li et al. 97. They reported the state of 20 famous cryptocurrencies including Bitcoin. They selected the top 20 cryptocurrencies on MarketCap. Beside the prices of the cryptocurrencies, their measurements also include the popularity metrics which is calculated based on their followers on Twitter and GitHub.

Significant interest from the researchers falls into prediction of the price of Bitcoin based on its popularity on the social platforms. For example, Abraham et al. 98 have presented a method to predict the price of Bitcoin and Ethereum based on Twitter and Google Trends data. They found that the tweet volume, rather than tweet sentiment, is a good predictor of the price direction. While, sentiment is proved to be invariably overall positive regarding the price direction. They built a linear model that takes tweets and Google Trends data as input, to accurately predict the direction of price changes. Similarly, Mittal et al. [99] correlated Bitcoin price predictions with Google search patterns and Twitter data. They believed that the sentiment in the blogs, emails and tweets can change human decision-making. By using linear regression,

polynomial regression, Recurrent Neural Network, and Long Short Term Memory 
based analysis, they found that there is a significant correlation of Bitcoin price change with Twitter data and Google trends data. Furthermore, Burnie et al. 100 have developed a new approach to temporalizing word2vec-based topic modelling that determines which topics on social media vary with shifts in the phases of a time series to understand potential interactions, i.e., relevant to the highly volatile Bitcoin price.

Some researchers predict volatility based on the volume. Balcilar et al. [101 have used a qualitative-based approach to find whether volume can predict Bitcoin returns and volatility.

In this work, we aim to study Bitcoin software ecosystem within a well-known collaborative platform (GitHub) that hosts millions of open-source projects. Furthermore, we plan to conduct a deep analysis of Bitcoin ecosystem's health considering the measures introduced by the research community of SEs health. Moreover, we are interested in developing and evaluating models that predict the health of the ecosystem.

\subsection{Mining GitHub}

GitHub being the leading collaborative development platform has been studied from different perspectives. The state-of-the-art research offers many studies related to mining GitHub data.

Kalliamvakou et al. 102 have identified a number of perils researchers should keep in mind while exploring GitHub repositories. Their study is around the main characteristics of a repository such as commit, pull-requests, and etc. They show that GitHub is a rich source of code, and mining GitHub can lead to interesting insights. They also found that GitHub is also used just for storing data and as a web hosting service. In another study, Kalliamvakou et al. [102] have conducted an online survey with GitHub users. They aimed to explore how GitHub is used for collaboration in 
commercial projects. They found that many commercial projects adopted practices that are more typical to open-source projects including characteristic such as reduced communication, more independent work, and self-organization. Furthermore, they discuss how GitHub's transparency and popular workflow can promote open collaboration, allowing organizations to increase code reuse and promote knowledge sharing across their teams.

Gousios et al. 103 have initiated the GHTorrent project. GHTorrent is a replica of GitHub REST API. It monitors the timeline of GitHub API and stores the raw JSON files in a MongoDB database. It also extracts the structure in a MySQL database. It includes all the features related to the repositories like timely metrics for commits, contributors, forks, watchers, issues, pull requests, etc. In another study, Gousios et al. [89 presented the dataset details and construction process and outlined the challenges and research opportunities emerging from this project.

In addition to studying GitHub itself, there are diverse studies on GitHub mining, such as identifying experts [104], analysing the discussions to explore the social norms among GitHub developers [105. Tsay et al. 105] claim that there is a lack of detailed understanding of the form and content of the discussions around pull requests. They performed a detailed analysis of a sample from the discussions around the pullrequests in GitHub. They selected the pull requests with extended discussions which reveal developers reasoning process. They used two methods to accomplish this, interviews with GitHub developers and content analysis of the pull-request discussion. They contributed with informed policies and tools that enable software developers to better manage open-source projects. Some researchers [106, 107] combine GitHub and Stack Overflow, the two leading social platforms, to bring some very interesting social and technical insights around open-source software development. 


\subsubsection{Mining Bitcoin Data}

There are various studies focused around cryptocurrencies including Bitcoin in GitHub. Trockman et al. [108] studied whether a cryptocurrency's market capitalization depends on its development (actively developed, high quality of code). They followed an econometric approach to answer these questions, using dynamic regression models for panel data with autoregressive errors and conducting Granger causality analysis. Using Granger causality tests, they found no compelling evidence for a dynamic relation between market capitalization and metrics such as daily stars, forks, watchers, commits, contributors, and lines of code changed. Their dataset includes 241 cryptocurrency projects on GitHub. Tonder et al. [109] created a dataset from GitHub including 7,000 repositories about different cryptocurrencies. The dataset is the foremost a quantitative measure of developer activity for building open source cryptocurrency development. The dataset includes data for 236 cryptocurrencies for 380 days (roughly from January 2018 to January 2019). They also collected cryptocurrency market capitalization, price, and volume from CoinMarketCap, currently one of the leading sites indexing cryptocurrencies. Zhauniarovic et al. [110] studied the use of Bitcoin for the purpose of donations to the open-source GitHub repositories. They analyzed the volume of donations over time. Furthermore, they evaluated the relationship of the amount of donations with popularity and age of the repository. They found a limited adoption of Bitcoin as a payment method for receiving donations, with nearly 44 thousand deposits adding up to only 8.3 million dollars in the last 10 years.

\subsubsection{Software Ecosystems}

Majority of the studies around identifying and studying the evolution of a software ecosystem is in the domain of open-source development. There are many studies 
that identify different ecosystems in GitHub. For example, Blincoe et al. [111] have identified 43 software ecosystems in GitHub. They proposed a new method, reference coupling, for detecting technical dependencies among the projects. Zhang et al. 112 proposed a new approach to detect similar repositories on GitHub based on the following three heuristics:

1. Repositories whose readme file contain similar content are likely to be similar with one another,

2. Repositories starred by users of similar interests are likely to be similar, and

3. Repositories starred together within a short period of time by the same user are likely to be similar.

Liao et al. 113 proposed a Topic-based Project Knowledge Metrics Framework (TPKMF). They used Latent Dirichlet Allocation (LDA) to construct topic-project relation matrix. They used TPKMF to create the project-relevance network and used Project-Spectral Clustering (CP-SC) algorithm to divide the project-relevance network.

Some researchers study specific ecosystems in GitHub. for example, Constantinou et al. 83] have studied social and technical permanent changes in Ruby ecosystem in GitHub.

Programming languages are also mostly favoured while identifying and studying a software ecosystem in open-source community. For example, Claes et al. 63] based their study on the challenges software ecosystems face in terms of dependency management. They explored whether use of GitHub influences R ecosystem in terms of distribution of packages and inter-repository package dependency management. The comprehensive R Archive Network (CRAN) contains official R repository and recompiled stable packages compatible with the recent version of $\mathrm{R}$ environment. According 
to the authors there are more than $74,000 \mathrm{R}$ packages. Their paper illustrated that many $\mathrm{R}$ packages hosted by GitHub are subject to inter-repository problems. The dataset used for the study contained R packages hosted on CRAN and GitHub. From the description file of a package they extracted the field of import packages and depends package for a package. Furthermore, they gathered the historical metadata about each package from CRAN and GitHub. They also interviewed five of the maintainers of $\mathrm{R}$ repositories on GitHub. They used experts views to confirm their views throughout the study. They provided actionable guidelines for $\mathrm{R}$ development community to deal the aforementioned problems. Some of the findings are as follows. The number of packages hosted on GitHub is increasing, while the packages hosted on CRAN are older. Majority of R developers use GitHub for development. They concluded their study with the following guidelines: the $\mathrm{R}$ package-based software ecosystem would strongly benefit from an automatic package installation and dependency management tool, like the ones that are currently available for other package-based software ecosystems.

Mens et al. [82] studied social and technical permanent changes in Ruby ecosystem in GitHub. They analyzed the co-evolution of a large number of base projects in this ecosystem over a period of nine years. The following are their main contributions: (1) modifications of the socio-technical network of the Ruby ecosystem in GitHub, (2) measured the impact on the ecosystem when contributors permanently abandon projects, (3) provided preliminary evidence about contributors migrating to different ecosystems, and (4) illustrated how data in GitHub can be used for socio-technical analyses of software ecosystems.

To the best of our knowledge, we are aware of no study that studies the Bitcoin software ecosystem and its health in GitHub or any other collaborative platform. 


\section{Chapter 4}

\section{Methodology}

As mentioned in the objectives this work (Section 1.3), we have divided our efforts into three main branches: categorization of the projects based on their contribution to the Bitcoin ecosystem, classification of the projects into different classes of health, and training classification models based on the labeled dataset to predict the health of the projects in the ecosystem. This chapter discusses the techniques we adopted in this research work. Section 4.1 explains the data collection process. Section 4.2 discuses the categorization of the projects, while Section 4.2.2, Section 4.2.3, and Section 4.2.4 explain the process of clustering, semi-supervised clustering, and category prediction, respectively. In Section 4.3, we define the health metrics and label the projects into three classes of health. Section 4.4 explains predictive modeling and analysis. Finally, Section 4.5 discusses the tools and libraries used in this study.

\subsection{Data mining}

A subset of the dataset used for this research comes from GHTorrent [89]. We retrieved a number of metrics such as the number of commits, issues, watchers, forks, contributors and programming languages from the GHTorrent dataset. However, GHtorrent does not store the data related to source code of the repositories. To 
collect source code features we used Pydriller [114 to clone each of the selected repositories and collect source code metrics.

\subsubsection{Project Selection and Preprocessing}

We used GHTorrent's MySQL database to identify Bitcoin related projects. We

queried the database using Google BigQuery [115] using the keyword "Bitcoin" in the name and description fields of a project. This query resulted to 80,453 projects. Further, we cleaned the dataset by applying the following filters:

1. We removed all "deleted" projects, as a result, the dataset was reduce to 74,628 projects.

2. We considered only "master" repositories. This filter reduced our dataset to a significant number, i.e., 16,833 projects. This shows that the majority of the Bitcoin projects are forks of the existing projects.

3. Furthermore, we considered projects with at least 10 commits. We ended up with 3,601 projects having more than 10 commits.

4. We focused on "active" projects. Thus, we selected the projects that have their last commit made in the last six months. This filter limits our dataset to only active or ongoing projects.

5. Our last filter eliminated non-solo projects, thus considering projects that are developed by more than two developers. This filter reduced the dataset to 581 projects.

The data collection process is illustrated in the Figure 2 . 


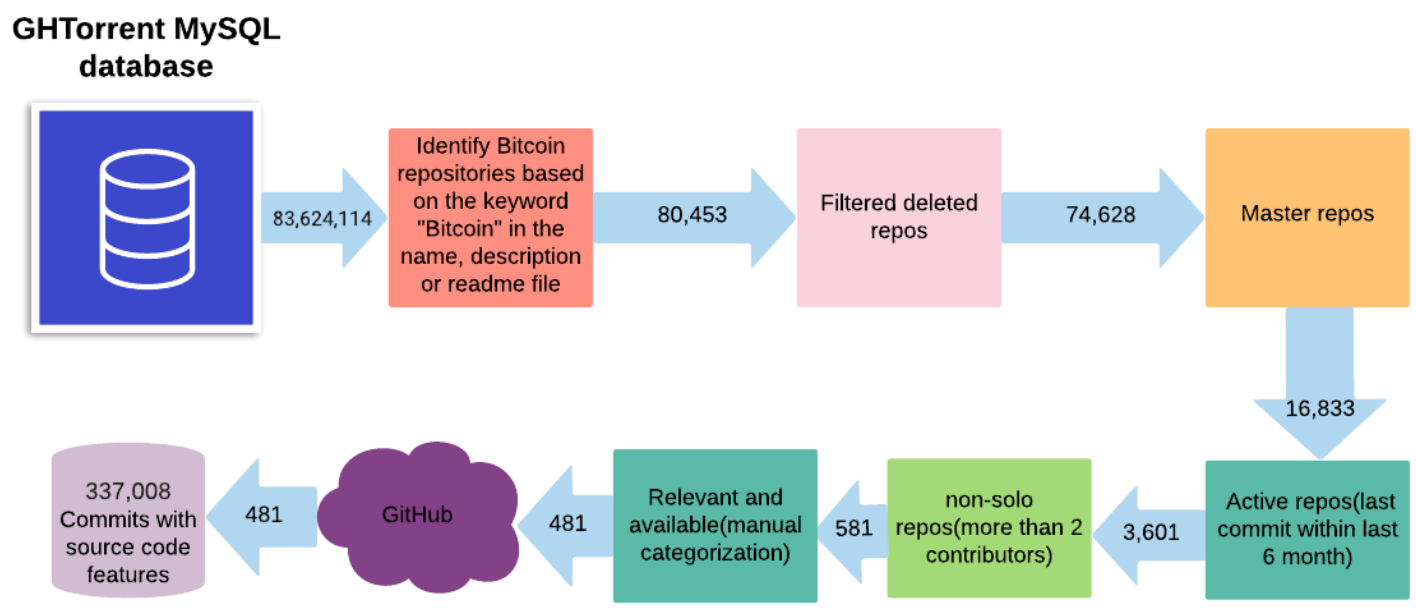

Figure 2: Data collection process.

\subsubsection{Collecting Source Code Features}

In order to analyze the quality of the source code, we need to extract features such as code complexity, methods count, token count, etc. To collect these code quality features we used pydriller to clone each repository in the dataset and extract the required features. Pydriller [114] is a Python framework which is used by the researchers to mine software repositories. By using this tool we retrieved cyclomatic complexity, methods count, token count, number of line, number of lines added, number of lines removed, and number of modifications for each commit. Out of 581 repositories 50 of them were not not found during the cloning process. We confirmed this by visiting the GitHub page for each repository, and found that these projects no longer exist. Furthermore, during our semi-automated classification of the repositories we found that 50 repositories are irrelevant, they are not Bitcoin repositories even though the name of the repository contains a "Bitcoin" word. Therefore, we have collected the source code features for 481 repositories over the period of 8 years. As result, there are 337,008 commits in the dataset for 481 repositories. Thus, we ended up having two final datasets: repo-level and commit-level. The repo-level dataset is comprised of 480 
projects with 24 features over the 8 years shown in Table 2. While the commit-level dataset contains 12 features reported in Table 3 over the period of 8 years.

\subsection{Defining the Bitcoin Ecosystem}

In this section, we define the Bitcoin ecosystem around its projects in GitHub. We aim to determine which applications of Bitcoin have open-source projects on GitHub. For this purpose, we will categorize the repositories in the dataset into different classes based on their contribution to the ecosystem. This will allow us to better understand the ecosystem. For categorization, we apply both semi-automated and data-driven approaches. The following sections discuss both approaches in detail.

\subsubsection{Manual classification of GitHub projects}

We studied the selected GitHub projects based on their name, description and README file. We started with the known Bitcoin ecosystem as discussed in Section 2.3.2. Initially, we started with development, wallet and payment categories. They are the prominent concepts in the Bitcoin ecosystem, so we assumed that they are present in GitHub. We confirmed this assumption by manually searching the GitHub website for Bitcoin projects which could be a part of a certain category. For

example, we knew that Bitcoin has its official repository on GitHub, and is a part of the development category. Similarly, for payment category, there are many businesses that are accepting Bitcoin as payment and have applications and likely host and maintain open-source projects on GitHub. Furthermore, after searching for Bitcoin wallets we were able to find that some very popular wallet applications such as Bitcoin Core wallet [33], Samourai [34], Armory [35] have GitHub projects. Thus, we categorized these projects in the dataset into the mentioned three categories. The process is semi-automated following two steps: 1) We used regular expressions to search for 
Table 2: Features in the dataset: repository-level.

\begin{tabular}{|c|c|c|}
\hline No & Feature & Description \\
\hline 1 & project_id & Unique ID for each repository \\
\hline 2 & name & Name of the repository \\
\hline 3 & URL & GitHub API URL of the repository \\
\hline 4 & description & Description for the repository \\
\hline 5 & language & The first 3 most frequent programming languages \\
\hline 6 & created_at & Date the repository is created at \\
\hline 7 & forked from & ID for its parent repository \\
\hline 8 & deleted & Whether repository is deleted or not \\
\hline 9 & updated_at & Date the repository is last updated \\
\hline 10 & forked_commit_id & ID of the commit when the repo was forked \\
\hline 11 & commit & Number of commits per repository \\
\hline 12 & issues & Number of issues per repository \\
\hline 13 & watchers & Number of watchers per repository \\
\hline 14 & contributors & Number of contributors per repository \\
\hline 15 & forks & Number of forks per repository \\
\hline 16 & cyclomatic complexity & Mean of CC over the commits in repository \\
\hline 17 & nloc & Mean of nloc over the commits in repository \\
\hline 18 & lines_added & Mean of lines added to repository over the commits \\
\hline 19 & lines_removed & Mean of lines removed from repository over commits \\
\hline 20 & token_count & Mean of token count over the commits \\
\hline 21 & method_count & Mean of method count over commits \\
\hline 22 & modifications & Number of modification per repository \\
\hline 23 & frq_change_type & Frequently changed type during the commits \\
\hline 24 & frq_file_changed & Frequently changed file \\
\hline
\end{tabular}


Table 3: Features in the dataset: commit-level.

\begin{tabular}{ll}
\hline Feature & Description \\
\hline project_id & Project ID \\
name & Name of the project \\
date & Date of the commit \\
cyclomatic complexity & Number of CC over the modifications in the commit \\
nloc & Mean of nloc over the modifications in the commit \\
lines_added & Number of lines added to the commit over the the modifications \\
lines_removed & Number of lines removed from the commit over modifications \\
token_count & Number of tokens over the modifications \\
method_count & Number of methods over modifications \\
modifications & Number of modifications for the commit \\
frq_change_type & Frequently changed type during the modifications \\
frq_file_changed & Frequently changed file during the modifications \\
\hline
\end{tabular}

keywords shown in Table 4 in the name or description of a repo. 2) Manually visiting the GitHub URL for the repositories to confirm the results.

During the initial search, we found that some of the repositories can be better presented by other categories other than the initial three categories that we stared with. Because they have very specific implementation, for example, the repositories in mining category are specific towards mining and payment, wallet or development can not represent these projects. Thus, we have identified six more categories of the ecosystem.

Our classification approach includes also some manual analysis of the repositories with no description or if the type of the repository was not clear from its description. For the repositories with no description (385 projects), we manually classified them using their README files.

In the following Section 4.2.2, we conduct a data-driven classification of the 
Table 4: Keywords.

\begin{tabular}{ll}
\hline Class & Keywords \\
\hline Development & $\begin{array}{l}\text { implementation,ide,protocol,plugin,module,algorithm,api,libraries, } \\
\text { tool,blockchain }\end{array}$ \\
\hline Wallet & $\begin{array}{l}\text { wallet,bitkey,xapo,opendime,wasabi,coldcard,greenaddress,sentinel, } \\
\text { electrum,Bitcoin core wallet,samourai,armory }\end{array}$ \\
\hline \multirow{2}{*}{ Services } & website,Bitcoin cash,proxy,optech,bitnode,otx,Bitcoin visuals, \\
& $\begin{array}{l}\text { blockstream,coindance,byll,lolli,fold,bazaar,tallycoin,bitpatron,bitrefill, } \\
\text { fastBitcoin,purse }\end{array}$ \\
\hline Documentation & documentation,doc \\
\hline Mining & $\begin{array}{l}\text { mining,miner,mine,honeyminer,pool,bitfury,canaan,bitmain,ebang, } \\
\text { halong mining,sluch pool }\end{array}$ \\
\hline Trade & $\begin{array}{l}\text { trade,exchange,bakkt,hodl hodl,localBitcoins,cash app,xbt provider, } \\
\text { paxful,cme group,bull Bitcoin,Bitcoin international trust }\end{array}$ \\
\hline \multirow{2}{*}{ Payment } & $\begin{array}{l}\text { payment,bitpay,opennode,blockstream satellite,paynym,azteco,btcpay, } \\
\text { gotenna }\end{array}$ \\
\hline Node & node,casa,nodl,dojo,raspiblitz,lightning,bitseed \\
\hline Data Analysis & data,analysis,social,media,analytic \\
\hline
\end{tabular}


repositories. We apply K-means clustering and compare the results from the semiautomated and data-driven approaches.

\subsubsection{Clustering}

In this section, we describe how we apply a clustering technique to find similar categories within the ecosystem. Clustering is an unsupervised machine learning technique which divides the sample points in a dataset into groups such that the points in a group are similar to each other but dissimilar to the points in the opposite groups.

\subsubsection{Data Pre-processing}

Outlier detection: We used z-score to find the outliers in the dataset. A z-score value of $+/-3$ is generally considered to be an outlier. We used this threshold, and as a result we removed 46 points from the dataset.

Z-score: A z-score describes the position of a raw score in terms of its distance from the mean. The z-score is positive if the value is above the mean, and negative if it is lower than the mean.

Data normalization: Furthermore, we standardize the features by using the following formula:

$$
\mathrm{X} \_s t a n d a r d=\frac{\mathrm{x}-\operatorname{mean}(\mathrm{x})}{\operatorname{std}(\mathrm{X})}
$$

Standardization of features is important for every machine learning task. This makes sure that features with values in different ranges have the same importance. For example, two features $\mathrm{x}$ and $\mathrm{y}, \mathrm{x}$ having values in range $1-100,000$ and $\mathrm{y}$ having values in range 1-20 does not make $\mathrm{x}$ more important. Standardization brings the features into the same range. 


\subsubsection{Feature Selection}

We include all the numerical features from Table 2 numbered 11-22 plus name and description of the repository. For name and description we included their TF-IDF values. We found that the Silhouette score, defined in Section 4.2.2.3, for clusters with and without name and description is almost the same. The silhouette score with these features is 0.772 , while without these features is 0.771 . Thus, we proceed with our final analysis without these feature.

TD-IDF values [116]: TF-IDF (term frequency-inverse document frequency) is a statistical measure that evaluates how relevant a word is to a document in a collection of documents. It is calculated by multiplying two metrics; how many times a word appears in a document, and the inverse document frequency of the word across a set of documents. TF-IDF is also used for extracting keyword from a text. The highest scoring word of a document are considered to be keywords for that document. We used it to extract the keywords from the name and description of the repositories.

\subsubsection{K-means clustering}

K-means 117 divides the data into $\mathrm{K}$ clusters and is the most widely used clustering algorithm. The number of clusters, K, should be provided as an input to the algorithm. The algorithm starts with $\mathrm{K}$ random centroids. A centroid is a data point in the dataset around which the cluster is formed. Iteratively, each data point in the dataset is assigned to a cluster based on its distance to the centroid. A data point is assigned to a cluster with minimum distance to the centroid. After the data points are clustered the mean of each cluster is taken and let that be the new centroid. The process is repeated (using the new centroids to form clusters, etc.) until the algorithm stops moving the centroids. There are different distance measures used by K-means such as, Euclidean, Cityblock (Manhatten), Correlation or Hamming. 
We use Euclidean distance, which calculates the distances between two real value vectors or two rows of data that have numerical values, such a floating point or integer values. Since the input features in this problem have numerical values, we use Euclidean distance with K-mean. We have also considered cosine similarity, but for normalized vectors cosine similarity and Euclidean distance are connected linearly. Also the resulted clusters from Euclidean distance with normalized data have higher Silhouette score than the clusters with cosine similarity. Therefore, we computed the final clusters with normalized data using Euclidean distance. We have also considered self-organizing maps [118] as clustering method. Yet, we found that this algorithm does not provide promising results for a small dataset. Self-organizing maps are best for high dimensional datasets [119].

Finding optimal K: Finding an optimal number of clusters or K is the fundamental step in unsupervised learning. We should find $\mathrm{K}$ such that adding another cluster does not give much better modeling of the data. We use the elbow method to find the optimal value for $\mathrm{K}$.

Elbow Method [120]: The elbow method plots values for K on the horizontal axis and the distortion on the $\mathrm{Y}$ axis. Distortion is calculated as the average of the squared distances from the cluster centers of the respective clusters. Typically, the Euclidean distance metric is used. When $\mathrm{K}$ increases, the centroids are closer to the clusters centroids. The improvements will decline, at some point rapidly, creating the elbow shape. This point the improvements decline is the optimal value for $\mathrm{K}$.

Silhouette Score [121]: We use silhouette score for cluster evaluation. It is used to measure the separation distance between two clusters. It is in the range of $[-1,+1]$, -1 means that the clusters are assigned in the wrong way and samples may have been assigned to wrong clusters. 0 means that the clusters are very near to the decision boundary, the distance between the clusters is insignificant. While +1 means that clusters are apart from each other. 
Table 5: Parameter optimization for decision trees.

\begin{tabular}{llll}
\hline \hline Model & Algo & tree_depth & F-score \\
\hline \hline Model 1 & Gini & $\mathbf{9}$ & $\mathbf{0 . 8 3 1}$ \\
Model 2 & Gini & auto & 0.8111 \\
Model 3 & Entropy & 9 & 0.765 \\
Model 4 & Entropy & auto & 0.790 \\
\hline \hline
\end{tabular}

\subsubsection{Semi-supervised Clustering}

Semi-supervised clustering [122] uses some knowledge which can be of two forms: pairwise-constraints or class labels for some of the data points. The class labels can also be converted to pairwise constraints. Pairwise constraints build a must-link and not-link relationship between the data points from the known labels. Since we have pre-defined nine categories after semi-automated categorization process. We can use some data points with class labels to learn pairwise constraints. We build Pairwise Constrained K-Means (PCK-Means) [123], providing the learned constraint and the number of clusters. We used $20 \%$ of the dataset to learn pairwise constraints and build the model with $\mathrm{K}=9$ and the learnt pairwise constraints. We applied the model to cluster the rest of $80 \%$ of the dataset.

\subsubsection{Category Prediction}

In this section, we build a decision tree classifier, discussed in Section 4.4.4, to predict the category of a software project. We used all the numeric features numbered as 11-24 in Table 2. We used 10-fold cross validation to find the optimal depth for the tree to avoid over-fitting. We also tuned the criteria parameter for the model. Table 5 shows the F1-score for different parameters. Based on the results we used Model1 for category prediction. 


\subsection{Health Analysis}

In this section, we define health metrics and classify the projects into different classes of health. Furthermore, we analyze the trends in the health for project level and analyze the health of the ecosystem as a whole. After classifying the projects into health classes we predict the project's health.

\subsubsection{Health Metrics Definition and Heuristics}

In order to classify the repositories we introduce four categorical variables into our dataset: i) Popularity, ii) Activity, iii) Complexity, and iv) Age. Furthermore, we assigned the features from the dataset which best describe the new category. We created the new categories for the purpose of simplicity, so we can easily label the data points. Table 6 shows the new categories, distinct classes in each category, features contributing to the category, and the algorithms through which the data points are classified.

Next, we offer a brief explanation for each category.

Activity: According to Brams et al. [30], repositories with a high number of source code commits are well developed. In addition, the high number of contributors is a proof that the open-source project is developed and maintained by multiple

developers. We also associate the number of issues created within a repository to the activity of the project, assuming that a high number of issues means more interest from the community towards the project.

Age: Age is another indicator used for determining the health of an open-source project. We prioritize the projects that are six or seven years old while are still being maintained by developers than those projects that are only a few months old. Thus, we include age as well in the process of labeling projects into health categories. 
Table 6: Metric calculated.

\begin{tabular}{llll}
\hline \hline Category & Labels & Contributing_features & Process_of_Labeling \\
\hline \hline Popularity & high & watchers & Algorithm 1 \\
& low & & \\
\hline \multirow{2}{*}{ Complexity } & high & corks & \\
& medium & nloc & Algorithm 3 \\
& low & lines_added & \\
& & lines_removed & \\
& & token_count & \\
Activity & medium & issues & Algorithm 2 \\
& low & contributor & \\
\hline Age & new & age_in_weeks & \\
\hline \hline
\end{tabular}


Popularity: From popularity and activity categories, we can determine how a project is performing socially. We include forks and watchers as the popularity metrics to our dataset. We believe that more popular projects are more likely to be actively developed and maintained. This belief is influenced by the literature, for example, according to Jansen et al. [1] if a project has more downloads it can predict the good health of the project. Furthermore, according to a study by Borges et al. [124], three out of four users consider the number of stars for an open-source project before contributing to it. The results from their study show that stars are viewed by contributors as the most useful measure of popularity on GitHub, with $83 \%$ of answers with scores $3(31 \%)$ or $4(52 \%)$. It is followed by forks with $72 \%$ of answers with scores $3-4$ (35\% and 37\%, respectively) and by watchers with $67 \%$ (37\% and $30 \%$, respectively).

Code Quality: From the features in the code complexity category, we can determine how a project is performing technically. If the complexity of the source code is high, it may contribute to a poor quality of code [125]. The complexity of source code can impact testability 126 of a program 125,127], thus, programs with good quality of code are easy to test [128]. In order to measure the code complexity, we choose cyclomatic complexity [127] because it is easy to calculate and interpret the complexity of the source code.

Cyclomatic Complexity: Cyclomatic complexity (CC) is a source code complexity measure that is directly correlated with the number of errors in the code. It is calculated through building a control flow graph of the code. Through this graph, CC measures the number of linearly independent paths in the code. Thomas McCabe [129] developed this metric for procedural languages, but it also supports object-oriented languages as well. According to Giuliano et al [125], a module's CC value directly correlates with the number of test cases necessary. Moreover, CC is simple to calculate and easy to understand to understand. A high CC thus implies 
Table 7: Metrics calculated.

\begin{tabular}{lcccccc}
\hline Feature & Min & 1st quartile & 2nd quartile & 3rd quartile & Mean & Max \\
\hline Commits & 12 & 44.5 & 342 & 914.3 & 623.86 & 10,901 \\
Issues & 0 & 2.25 & 70.06 & 527.73 & 111.12 & 13,038 \\
Contributors & 2 & 2.35 & 5.19 & 128.28 & 6.5 & 442 \\
Watchers & 0 & 10.45 & 122.53 & 778.41 & 223.06 & 32,056 \\
Forks & 0 & 6.39 & 47.97 & 538.94 & 82.95 & 1,4951 \\
Age & 0.43 & 15.79 & 48.03 & 51.56 & 51.63 & 380 \\
CC & 0 & 4.51 & 30.23 & 211.54 & 34.72 & $1,929.77$ \\
\hline
\end{tabular}

high critically, and the code will have a higher defect density in comparison to the code with a relatively lower CC; test effort is higher and maintainability severely reduced.

Labeling: Using quartiles as thresholds is a common practice by the researchers in different fields [130, 131]. Shih et al., [131], used quartile analysis for automatic threshold determination in an image segmenting task. Similarly, for labeling, we considered quantiles as the threshholds instead of the mean, because the mean can be affected easily by the outliers in the data. Furthermore, we want to classify the projects into three classes, so setting the thresholds with quantile for the classes is more meaningful and can divide the data effectively. The following algorithms show the labeling process of the data points. Table 7 shows the descriptive statistics for the features in the ecosystem. The Algorithm 1, Algorithm 2, Algorithm 3, Algorithm 4, Algorithm 5 show the process of classification.

Algorithm 1 shows the process of categorizing the projects based on the popularity metric, i.e., watchers and forks. If the values of both these features are greater than the first quartile then we label the project's popularity as "high". If the value for either watchers or forks is greater than the third quantile, then we label the project as highly popular as well. While if any of the features have a value less than the first 
quantile then we label the project as having "medium" popularity. If the values for watchers and forks are less than the first quartile then it's popularity is labeled as "low".

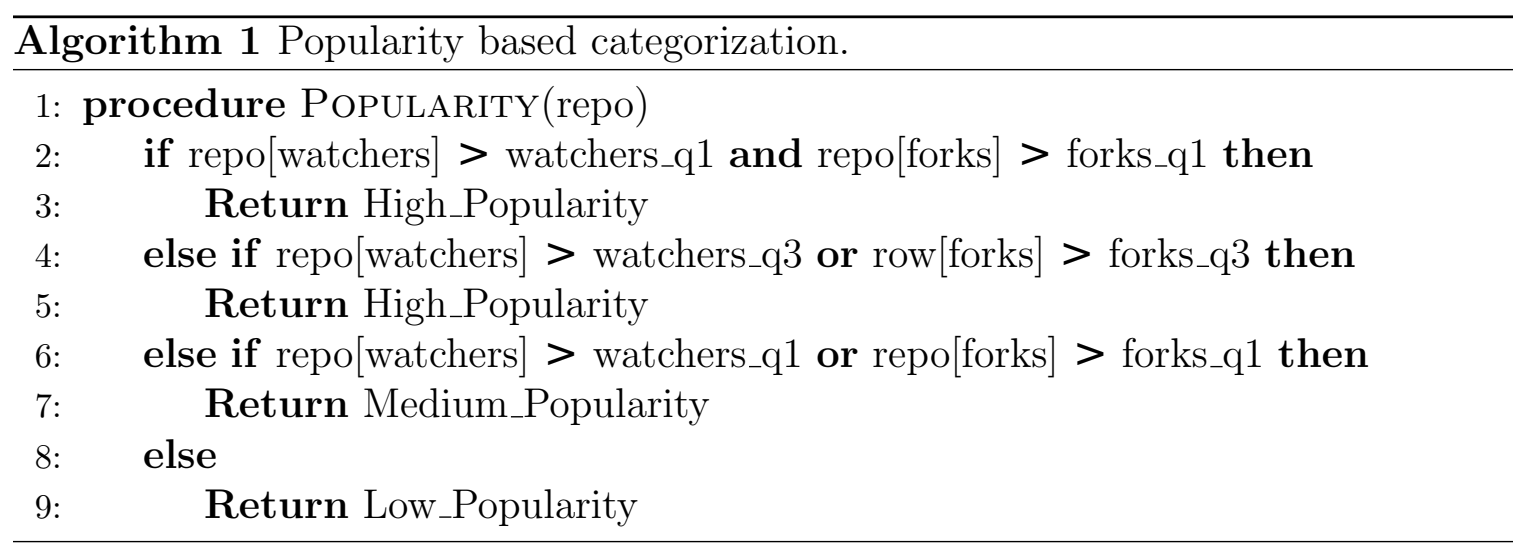

Algorithm 2 shows the classification based on the activity of a project. If the values of all the features are greater than the first quantile, then we label the project's activity as high. Furthermore, if two of the features have a value greater than the thirst quantile we label the project as having high activity. On the other hand, if any of the features have a value less than the first quantile, then we label the project as low. For the class medium acitivity; two of the features should have a value greater than the first quantile.

Algorithm 3 shows the classification based on the age of the repositories. We set two thresholds for this category. If the value of age is less than the first quartile then we label the repo as a new project. While if any of the features have a value less than the first quartile then we label the repo as growing. On the other hand, if both of the values are less than the first quartile then we label the repo as mature.

Similarly, Algorithm 4 shows the process of classification of projects based on the complexity of the source code. Here again, we set three thresholds. First, if the value of the complexity is less than the first quartile then we label the project as low in terms of complexity, but if the value is between the first quartile and second we 

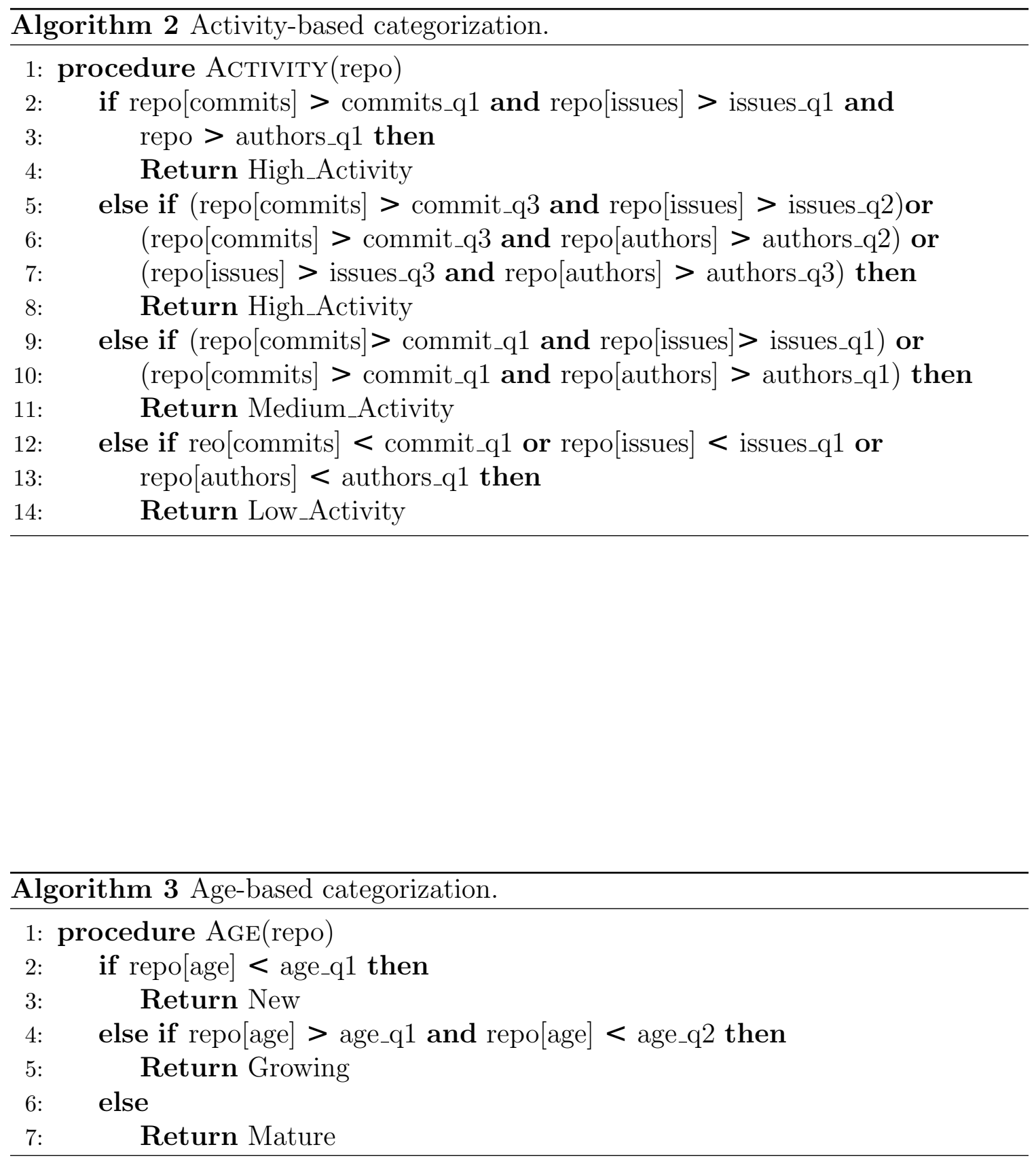
the project is labeled as medium complexity. Third, if none of the above options then the repo is classified as having high complexity.

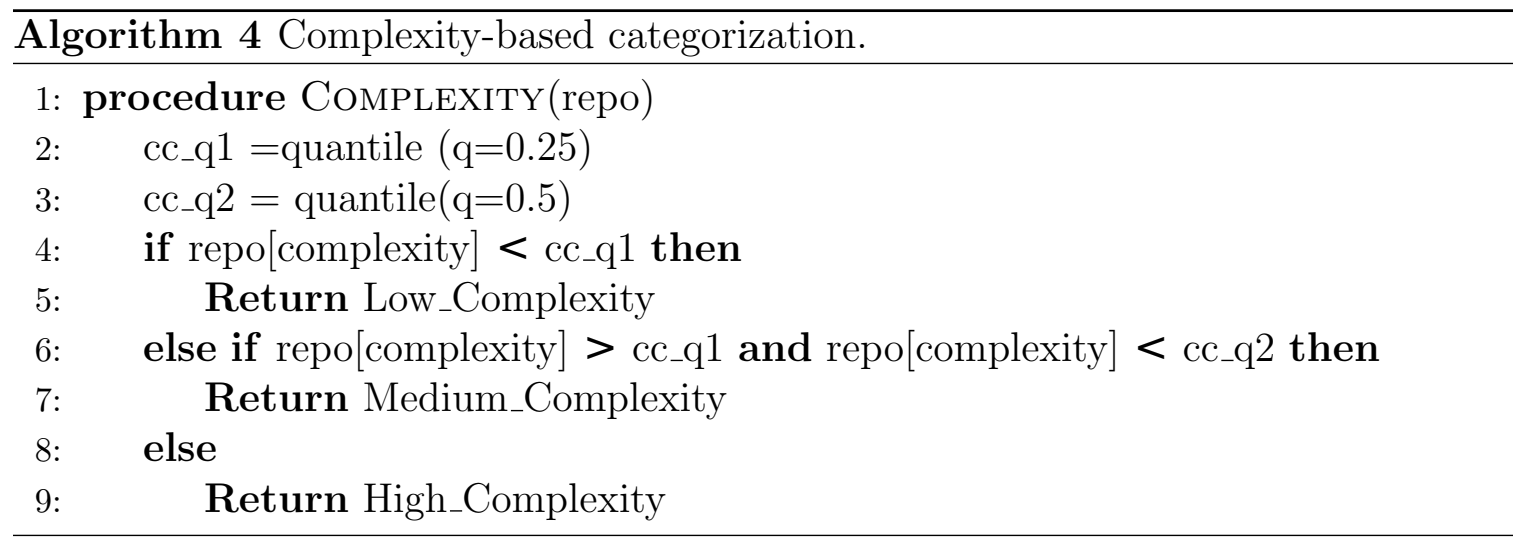

Finally, we combine the new categories and classify projects into three categories of health. While health can be defined as spectrum of different categories, we classify project's health into "healthy", "low risk", and "at risk" categories. Algorithm 5 shows the process of classification based on project's health. If a project shows "low" activity, defined as "new", with "low" popularity, "high" or "medium" complexity, then we classify the project as At Risk. For the second class, if a project demonstrates "high" or "medium" activity, is a mature or growing project, with "high" and "medium" popularity, with "low" and "medium" complexity values, we label the project as Low Risk. If none of the above conditions are satisfied, then the repository is classified as Healthy.

\subsubsection{Trends in Health of the Ecosystem as a Whole}

We define the health of a software project in terms of four qualities such as popularity, activity, maturity (age), and code quality. These measures combine both technical and social aspects related to the health of an open-source project. In this section, we perform a quantitative analysis of the trends considering all the defined health metrics for the ecosystem as whole, not just at the project-level. 


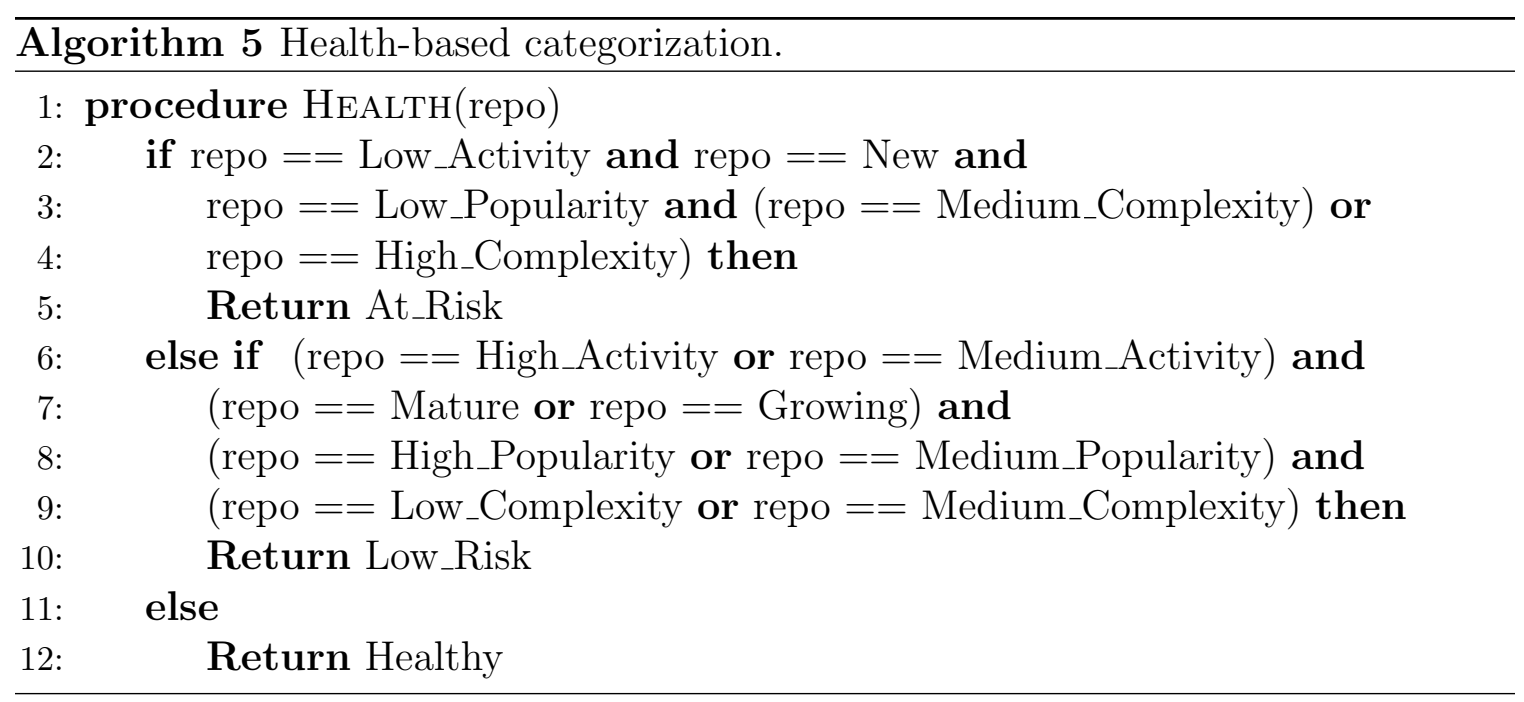

Activity: To identify the trends of the ecosystem's health over the years we did following:

1. For the analysis of the contributions, we consider the number of commits per year, per category, and a combination of both, as well as the change in contribution per year.

2. According to De Laat 132 a continuous collaboration between different stakeholders is important for an ecosystem to function well. To analyze collaboration between developers, we analysed the number of authors working in one, two, three, four and five or more projects. For retention rate, we calculate the number of new authors subtracted from the loss of authors every year. We calculate the change of contributors for all the projects per category, per year and per category.

3. To determine the loss of contributors in a year, we consider the authors whose last commit was made in that year.

4. To analyze issues, we determine the average number of issues created each year for all projects and the average number of issues created for each year for all 
categories.

Popularity: To analyze the popularity, we calculate the mean of watchers and forks for each year for all repositories, as well as the average number of watchers and forks per year and per category.

Code Quality: To analyze the quality of the source code, we determine how the quality of the $\mathrm{CC}$ is changing over the years.

\subsection{Predictive Modeling and Analysis}

In this section, we conduct a comparative study to predict the health of software projects based on the features we have in our dataset. As discussed in Section 4.3, we classified the repositories in our dataset into three different classes. We will use this for training different classification models and evaluating which one of them performs better when predicting the health of a project in the ecosystem.

Classification [133 is a supervised machine learning algorithm. It is a technique to categorize the dataset into distinct categories. It has a vast variety of applications, for example, text classification, speech recognition, handwriting recognition, etc. Classifiers can be binary or multi-class classification. Binary classification categorizes the data into two classes. While in multi-class classification we have more than 2 classes. In this research, we are focused on multi-class classification, because we have three distinct classes in our dataset. The following are some concepts that we briefly discuss as they are used throughout this section.

Loss function: The loss function calculates the difference between the actual value for every data point in the training dataset and the predicted value by the model. The average of the values returned by the loss function is determined as the cost function. The computed value is the error value and is directly proportional to the difference between the actual values for the data points and predicted values 
by the model. Different models use different loss functions, for example, logistic regression uses the sigmoid function to calculate the difference, while SVM uses hinge loss function.

Regularization [134: Regularization is the trade-off between the complexity of the model and the loss function. If the model is too complex it would lead to overfitting, and over-fitted models do not perform well on unseen data. On the other hand, if the model is too simple, it would demonstarte a poor accuracy. It prevents the model from overfitting. There are two kinds of regularization in machine learning.

1. L1 Regularization — Lasso Regularization: L1 Regularization or Lasso Regularization adds a penalty to the error function. The penalty is the sum of the absolute values of weights.

$$
\operatorname{Min}\left(\sum_{i=1}^{n}\left(y_{i}-w_{i} x_{i}\right)^{2}\right)+p \sum_{i=1}^{n}\left|w_{i}\right|
$$

, where $\mathrm{p}$ is the tuning parameter which decides that how much we want to penalize the model.

2. L2 Regularisation - Ridge Regularization : L2 Regularization or Ridge Regularization also adds a penalty to the error function. But the penalty here is the sum of the squared values of weights.

$$
\operatorname{Min}\left(\sum_{i=1}^{n}\left(y_{i}-w_{i} x_{i}\right)^{2}\right)+p \sum_{i=1}^{n}\left(w_{i}\right)^{2}
$$

, $\mathrm{p}$ is the same as in L1 Regularisation.

Gradient descent: 135 Gradient descent is an optimization algorithm used to find the values of parameters (coefficients) of a function (f) that minimizes a cost function (cost). The goal of this algorithm is to change different values for the 
coefficients and reach minimal loss for the model. Gradient descent is best used when the parameters cannot be calculated analytically (e.g., using linear algebra) and must be searched for by an optimization algorithm.

Cross validation [136]: Cross validation is a one of the re-sampling methods, which try to assess the generalization ability of a model on real data and avoids overfitting.

Confusion matrix: [137] Confusion matrix is a table representing the prediction results for a classification model based on test data. Most of the evaluation metrics such accuracy, F1-score, precision, recall, specificity, sensitivity, and ROC curve are calculated from the confusion matrix.

\subsubsection{Preparing Data for Training Models}

Balancing: For classification problems it is important to balance the dataset, meaning that there should be similar number of data points in each class. The dataset which we labeled in the previous section, resulted in an imbalanced dataset. We have 322, 85, 74 data points for the class Low Risk, Healthy and At Risk, respectively. To balance the dataset we up sampled the minority classed in our dataset. We also test the models with a down sampled dataset, where we down sampled the the majority class. But we have achieved better results with up sampling.

Data normalization: We used the Standard scalar class from scikit-learn to normalizes our dataset.

Outlier detection: We calculated z-score for the features to detect outliers, since the number of outliers is not significant, we removed them from the dataset. We used z-score class for data pre-processing from scikit-learn. We set the threshold for the z-score to 3. We removed the points with a $\mathrm{z}$-score greater than the threshold. 


\subsubsection{SVM}

Support Vector Machines (SVM) 138 is a machine learning algorithm which separates classes with a hyperplane. Hyperplanes are decision boundaries to classify the data. The data point falling on either side of the hyperplane can be attributed to a different class. The data points that are near the boundaries are called support vectors, and they influence the orientation of the hyperplane.

SVM is highly preferred by data scientists because it achieves higher accuracy with less computation. It can be used for both regression and classification tasks, but mostly it is used for classification. The objective of this algorithm finds the best hyperplane in $\mathrm{N}$-dimensional space (N- features) to distinctly classify the data points. There will be many planes that can divide the points, but it aims to find a hyperplane with maximum margin. Margins are the distance between the points from the opposite class. The number of features in the dataset is the number of dimensions of the hyper plane. If there are two features in the dataset the hyperplane is a line. If it is more than two, it is a hyperplane.

Loss function: In SVM we try to maximize the margin to minimize the loss. For this purpose, we use Hinge Loss. The following is the formula to calculate the Hinge Loss:

$$
c\left(x, y, f(x)= \begin{cases}0 & \text { if } \quad y * f(x)>=1 \\ 1-y * f(x), & \text { else }\end{cases}\right.
$$

We can also add the regularization parameter to balance the margin maximization and loss or to prevent models from overfitting. After adding the regularization 
parameter the hinge loss function looks as follows.

$$
\operatorname{Min}_{w} \lambda\|w\|^{2}+\sum_{i=1}^{n}\left(1-y_{i}\left\langle x_{i}, w\right\rangle\right)_{+}
$$

The difference between SVM and other classifiers is the kernel trick. If the data point is not linearly separable it amps the points in a higher dimension, where they can be distinctly separable. There are many kernels that are used according to different problems and detests. For example, polynomial kernel, Gaussian kernel, Linear kernel, Gaussian radial basis function (RBF), Laplace RBF kernel, Hyperbolic tangent kernel, Sigmoid kernel, Linear splines kernel in one-dimension, ANOVA and radial basis kernel. We discuss the following two kernels which are used in this study:

1. Linear kernel: For linear kernel the equation for prediction for a new input using the dot product between the input (x) and each support vector (xi).

2. Gaussian radial basis function (RBF): RBF uses normal curves around the data points, and sums these so that the decision boundary can be defined by a type of topology condition such as curves. It is a general-purpose kernel; used when there is no prior knowledge about the data.

We used SVC class from scikit-learn which implements on-vs-all approach for multi-class classification. Furthermore, we iterative train the model on different parameters to choose the parameters with the best weighted F-score. The models are trained with 10 -fold cross-validation. Table 8 shows the weighted F1-score for different parameters. Accordingly, we used $\mathrm{C}=1000$ with a linear kernel.

$\mathrm{C}$ is the penalty for miss-classifying a data point. When $\mathrm{C}$ is small, the classifier is okay with miss-classified data; it shows high bias and low variance. While if $\mathrm{C}$ is high, the classifier is heavily penalized for miss-classifying, and it shows low bias and high variance. Gamma is the spread of the kernel and therefore, the decision region. When 
Table 8: SVM models with different F1-scores.

\begin{tabular}{lcccc}
\hline \hline Model & Kernel & C & Gamma & Weighted F-score \\
\hline \hline Model 1 & RBF & 1 & 0.001 & 0.338 \\
Model 2 & RBF & 1 & 0.0001 & 0.173 \\
Model 3 & RBF & 10 & 0.001 & 0.488 \\
Model 4 & RBF & 10 & 0.0001 & 0.354 \\
Model 5 & RBF & 100 & 0.001 & 0.654 \\
Model 6 & RBF & 100 & 0.0001 & 0.472 \\
Model 7 & RBF & 1000 & 0.001 & 0.731 \\
Model 8 & RBF & 1000 & 0.0001 & 0.582 \\
Model 9 & Linear & 1 & - & 0.682 \\
Model 10 & Linear & 10 & - & 0.723 \\
Model 11 & Linear & 100 & - & 0.730 \\
Model 12 & Linear & $\mathbf{1 0 0 0}$ & - & $\mathbf{0 . 7 3 8}$ \\
\hline \hline
\end{tabular}

the Gamma is high, the decision curve of the decision boundary is high which creates islands of decision boundary around data points. On the other hand, if Gamma is low, the decision curve is very low and hence, the decision boundary of the classier.

\subsubsection{KNN}

The assumption behind the K-Nearest Neighbor (KNN) [139] is that similar things exist in close proximity. It calculates the similarity by calculating the distance between the data points. The critical point in this algorithm is choosing the right number for $\mathrm{K}$. To select the $\mathrm{K}$ that is right for your data, we run the KNN algorithm several times with different values of $\mathrm{K}$ and choose the $\mathrm{K}$ that reduces the number of errors we encounter while maintaining the algorithm's ability to accurately make predictions when it's given data it has not seen before. 
In order to find the optimal value for $\mathrm{K}, \mathrm{p}$, and leaf_size we apply 10-k crossvalidation with GridSearch. We select the value which minimises the cross-validated score. From the GridSearch we get $\mathrm{p}=1, \mathrm{k}=1$, and leaf_size $=1$.

$\mathrm{K}$ identifies the numbers of neighbors to consider when labeling a new data point. We provide the GridSearch a range of values between 1 to 30 for $\mathrm{K}$. The distance used by the model is identified by $\mathrm{p}$, if $\mathrm{p}=1$, the algorithm is using Manhattan distance, otherwise if $\mathrm{p}=2$, the algorithm is using Euclidean distance. We only consider Manhattan and Euclidean distances for choosing the optimal measure of distance. Furthermore, the value of leaf size affects the speed of construction and query, and in the GridSearch we provided a range of values from 1 to 50 .

\subsubsection{Decision Trees}

Decision trees [140 are another predictive model that covers both regression and classification problems. Decision trees are most commonly used in data mining, but it also has a vast number of applications in machine learning. In general decision tree algorithm is referred to as CART (Classification and Regression Tree). The algorithm is recursive in nature as it recursively splits the tree based on the same function. This is why it is also known as the greedy algorithm. Tree models are created through two main steps such as induction and pruning.

Induction: It is the process of creating the tree where we set all the hierarchies boundaries based on our dataset. It follows the Recursive Binary splitting strategy to split the tree. In this strategy, all the features are considered at different split points. At each level, a feature that causes a minimum cost is selected for the split.

Pruning: The accuracy of the tree can be increased by pruning the branches with features that have less importance. By using this method, we reduce the complexity of the tree and improve the predictive power of the tree by avoiding overfitting. There are the following two strategies for pruning: 
1. Reduced error pruning: In this method the pruning starts at the leaf level, each node with the most prominent class is removed, the change is kept if it does not deteriorate the accuracy of the model.

2. Weakest link pruning: This strategy is also known as cost complexity pruning, this is a more sophisticated method. It uses the learning rate to decide whether a node should be removed based on the subtree at a certain node.

Cost function: The following is a simple cost function for regression

$$
E=\sum(y-\text { prediction })^{2}
$$

, where $\mathrm{y}$ is the actual value for the data point and prediction is the value predicted by the model. For classification problems, there are many methods, for example, the Gini score, or entropy. The following is the formula for Gini score:

$$
E=\sum\left(p_{k}\right) *\left(1-p_{k}\right)
$$

Where $p_{k}$ is the proportion of training instances of class $\mathrm{k}$ in a particular prediction node. A node should ideally have an error value of zero, which means that each split outputs a single class $100 \%$ of the time. This is exactly what we want because then we know, once we get to that particular decision node, what exactly our output will be and whether we are on one side of the decision boundary or the other.

Since the performance of decision tree depends on the attribute selection method and the depth of the tree, we iterative trained some models to choose the best hyper parameters. We do tree pre-pruning parameter optimization by limiting the depth of the tree for the value 3. Since our dataset is small limiting the dept of the tree is decreasing the F-score for the model. Table 9 shows the F-score for different parameters. Based on the results we used Model 2 with parameters Criterion $=$ Gini 
Table 9: Parameter optimization for decision trees.

\begin{tabular}{llll}
\hline \hline Model & Algo & tree_depth & F-score \\
\hline \hline Model 1 & Gini & auto & 0.970 \\
Model 2 & Gini & auto & $\mathbf{0 . 9 7 9}$ \\
Model 3 & Entropy & 3 & 0.953 \\
Model 4 & Entropy & 3 & 0.957 \\
\hline \hline
\end{tabular}

and tree_depth $=$ auto in our evaluation of the models.

\subsubsection{Naive Bayes}

Naive Bayes [141] is a probabilistic classifier based on Bayes theorem. It assumes that the variables are conditionally independent.

$$
\begin{gathered}
p(c \mid x)=p(x \mid c) p(c) / p(x) \\
p(c \mid x)=p\left(x_{1} \mid c\right) \times p\left(x_{2} \mid c\right) \times p\left(x_{3} \mid c\right) \ldots p\left(x_{n} \mid c\right)
\end{gathered}
$$

The above formula is the foundation of Naive Bayes algorithm. It finds the probability of an event $p(c \mid x$ based on the prior knowledge that may relate to that event $p(x)$. It finds the maximum posterio which is maximal $p\left(c_{i} \mid x\right)$ with the above assumption applying to Bayes theorem in order to do classification. The following are the parts of Naive Bays algorithm:

1. $p(x \mid c)$ - Posterior Probability The conditional probability of the response variable (target variable) given the training data inputs.

2. $p(x)$ - Prior Probability The probability of the response variable (target variable).

3. $p(c)$ - Evidence The probability of the training data. 
4. $p(c \mid x)$ - Likelihood The conditional probability of the training data given the response variable.

This assumption reduces the cost of computation because it is counting the distribution of the class only. Even though the assumption is not valid in most cases since the attributes are dependent, surprisingly Naive Bayes is able to perform impressively. Navies Bayes are simple and easy to implement. This algorithm needs a small training dataset and performs efficiently on large datasets.

There are three types of Naive Bayes classifiers.

1. Multinomial Naive Bayes: This technique is mostly used for document classification. The features/predictors used by the classifier is the count of words present in a document.

2. Bernoulli Naive Bayes: This is similar to multinomial, but the out or the dependent variable is binary. In this, the classifier categorizes the data into two distinct classes.

3. Gaussian Naive Bayes: When the features are continuous values not discrete, we assume that they are taken from a Gaussian or normal distribution. Since we have only numeric and continuous features in our dataset we chose Gaussian as the Kernel Function for our model.

Training and testing the model For training and testing we split the data into 80, 20 percent for training and testing sets, respectively. We run the model with $10 \mathrm{k}$ cross validation technique. We trained the Naive Bayes model with GuassianNB with default parameters. We choose GuassianNB because the input features are continuous variables. 
Table 10: Confusion matrix.

\begin{tabular}{l|lll}
\multicolumn{3}{c}{ Actual Values } \\
\hline \multirow{3}{*}{ Predicted Values } & Yes & TP & FP \\
No & FN & TN
\end{tabular}

\subsubsection{Model Evaluation}

In classification algorithms, the matrix which shows how many samples are in the right class and how many is in the wrong class is called the confusion matrix. Its structure is shown in the Table 10 .

From the confusion matrix of a classifier we can discuss about the performance (accuracy) of the classification model using the following terms:

1. TN (True Negative): When a case is negative and predicted negative.

2. TP (True Positive): When a case is positive and predicted positive.

3. FN (False Negative): When a case is positive, but predicted negative.

4. FP (False Positive): When a case is negative, but predicted positive.

There are many metrics based on which we can evaluate the performance of a classification algorithm. The most important ones are accuracy, precision, F1-score, recall, specificity and ROC. All of them are based on the confusion matrix of the classifier.

Accuracy: Accuracy of a classifier is the percentage of number of observations that are correctly classified. The following equation calculates the accuracy.

$$
\text { Accuracy }=\frac{T P+T N}{T P+F P+T N+F N}
$$


Recall: Recall or sensitivity indicates the correct positive values in the total number of positive samples.

$$
\text { Sensitivity }=\frac{T P}{T P+F N}
$$

Precision: Precision identifies how accurately the model predicted the positive classes.

$$
\text { Precision }=\frac{R P}{T P+F P}
$$

Specificity: Specificity (true negative rate) measures the rate of actual negatives identified correctly.

$$
\text { Specificity }=\frac{T N}{T N+F P}
$$

F1-score: F1-score is the weighted average score of recall and precision. The value at 1 is the best performance and at 0 is the worst.

Receiver Operator Curve (ROC): The ROC plots TPR against FPR at various threshold settings. This is the best method to compare the performance of multiple classifiers. The true positive rate is also known as recall, sensitivity, or probability of detection. The false-positive rate is also known as the fall-out (1-specificity). Accuracy is measured by the area under the ROC curve (AUC). An area of 1 represents a perfect model and an area of .5 represents a worthless model. The higher is the AUC, the better is the model in predicting 0's as 0 and 1 as 1 . When the value of $\mathrm{AUC}$ is near to 0 it reciprocates class, e.g., if $\mathrm{AUC}=0$ then it predicates 0 as 1 and 1 as 0.

We use the classification results and ROC curve to evaluate the performance of the classification models. The classification results shows the main accuracy, precision, recall, and F1-score on a per-class basis. It also calculates the macro average and weighted average for every metric except accuracy. The reported averages include 
macro average which is averaging the unweighted mean per label, the weighted average is averaging the support-weighted mean per label.

\subsubsection{Model explainability}

In this section, we determine which features are used by the models to predict the outcome. We use permutation feature importance to identify the features that are considered as important by the model. We calculated permutation importance after a model is fitted and on the test data. We used eli5 [142], a scikit-learn library for calculating permutation importance and apply AUC score as a measure of performance.

Permutation Feature Importance: Permutation feature importance [143] is a model inspection method. It calculates the importance of a feature by calculating the increase in an estimator's prediction. It randomly selects a feature by shuffling its values. A feature is "important" if shuffling its values results into an increase in the model's error. Because, in this case the model relied on this feature to predict the outcome. A feature is "not important" if shuffling its values does not change the model's error.

\subsection{Tools and Libraries Used}

Pydriller [114]: We used Pydriller to collect the source code features, and calculate the cyclomatic complexity. This tool clones a repository, collects the commits and calculates the metrics. It requires the repository's HTML URL, the start and the end dates to collect the commits. It calculates the features for each commit by iterating over all the modification of a commit. Pydriller uses Lizard [144] to calculate to calculate the source code features. Lizard is a library which analyzes cyclomatic complexity of the source code. It supports almost all the popular languages such as C/C++, Java, C\#, JavaScript (With ES6 and JSX), Objective-C, Swift, Python, 
Ruby, TTCN-3 and more.

Scikit-Learn [145]: is a machine learning library for Python. It is build on top of some most popular technologies such as Pandas, Numpy, and matplotlib. It has almost all the functionalities needed in data science tasks, for example, preprocessing, unsupervised and supervised machine learning algorithms which includes, but not limited to regression, classification, clustering and model selection. We used scikit-learn machine learning algorithms such as SVM, CART, KNN and logistic regression for the predictive analysis of the repositories' health. In addition we used preprocessing and normalization for preparing the data and models.

Pandas [146] is an open source data analysis tool build on top of Python. It works very efficiently with large datasets by providing fast indexing techniques. We used the data frame object of this library through out the analysis. Furthermore, we used it for reading and writing SQLite DB, and csv files. We also used this tool for cleaning and manipulating the data, for example merging the dataset from different sources such as GHTorrent and Pydriller.

NumPy 147 is a scientific package for Python. Beside string operations it provides a range of other powerful operations such as an N-dimensional array object, sophisticated (broadcasting) functions,and useful linear algebra. We used the Ndimensional array object during different sections for calculating metrics such source code quality metrics.

Regular expressions are a special text string for describing a search pattern. We used re, a Python library identifying Bitcoin repositories in GHTorrent and categorizing the repositories based on their name, description and README files.

Seaborn 148 is a data visualization library for Python based on matplotlib. We used different plots such as scatter, bar, heatmap, density plots for data visualization.

SQLite and CSV We used SQLite database and csv files for data storage. SQLite is a c-language library that implements a small, self maintained SQL engine. Python 
has sqlite3 package to work with SQLite engine. Comma separated values file is a plain text file, which stores values in different forms such as numbers, strings, etc. in a structured tabular form. Python's Pandas package works very efficiently with csv files.

Google BigQuery [115]: We used Google BigQuery to query the GHTorrent SQL dataset.

Colaboratory [149]: We used Colaboratory for the development with Python programming language. Colaboratory is a Google-based product which hosts Jupeter notebook services. It is feasible for machine learning and data analysis tasks.

Linux server We used a Linux server to run Python scripts for mining GitHub data. 


\section{Chapter 5}

\section{Analysis and Results}

This chapter presents the results and answers to the research questions from the Section 1.3. In Section 5.1, we present the results of our manual classification and clustering to answer RQ1. Section 5.2 explains the results of the health analysis (RQ2), and Section 5.4 presents the results of the analysis of trends. Section 5.4 discusses the results of predictive modeling (RQ4).

\subsection{RQ1: What Categories Are Representing the Bitcoin Ecosystem in GitHub?}

\subsubsection{Results of Manual Categorization}

After a thorough research about the applications of Bitcoin, we categorized the selected projects into nine major categories. Table 11 presents the descriptive statistics of the categories that we found to represent the Bitcoin ecosystem in GitHub.

During this manual classification, 50 out of 581 projects (8\%) were excluded from the dataset as they were determined to be irrelevant. These were not Bitcoin related projects. We now offer a brief explanation of each category: 
Table 11: The categories in the Bitcoin software ecosystem.

\begin{tabular}{c|cccccc}
\hline Category & \#repos & \#issues & \#watchers & \#devs & \#commits & Languages \\
\hline Data analysis & 25 & 95 & 103 & 80 & 1,539 & Javascript,Python,Swift \\
Development & 223 & 32,114 & 79,050 & 1,661 & 181,875 & C++,Python,batchfile \\
Documentation & 6 & 17 & 23 & 17 & 186 & None \\
Mining & 11 & 41 & 58 & 36 & 2,116 & Groff,Shell \\
Node & 26 & 6,952 & 10,030 & 421 & 28,065 & Python,C++,Javascript \\
Payment & 27 & 626 & 1,067 & 84 & 8,580 & HTML,Javascript,CSS \\
Services & 86 & 5,366 & 6,153 & 387 & 26,023 & CSS,Javascript,HTML \\
Trade & 31 & 2,180 & 5,501 & 180 & 14,578 & Java,Elixir \\
Wallet & 46 & 6,062 & 5,309 & 291 & 34,173 & Java,Python,Ruby \\
\hline
\end{tabular}

Development: Any repository which contains specific implementation of a protocol, IDE, plugin, module, library, API, tool, or algorithm is classified as development. This category has the highest number of projects and commits - 223 and 181,875, respectively. Bitcoin's main project, called bitcoin/bitcoin [150], is a part of this category, which is the most active project in terms of collaboration, popularity and age in the ecosystem. The second representative of this category is CCTX (CryptoCurrency eXchange Trading Library) [151], which is a trading API. Moreover, 1,661 unique developers are contributing to this category. The top three programming languages in this category are JavaScript, Python and Swift.

Documentation: The projects containing Bitcoin documentation are classified under this category. There are only six projects that contain documentation for Bitcoin. One of the projects in this category explains the security threat model of the Bitcoin cryptocurrency [152]. Besides, 17 unique developers are contributing to this category.

Services: Any specific implementation of any kind of service is classified under this class. For example, there are different online resources that offer a variety of services related to Bitcoin and its information. Furthermore, many businesses are 
adopting Bitcoin as payment, they host their websites to provide any needed information related to their business. For example, Bitcoin Visuals [153 provides the following services related to Bitcoin: 1) makes Bitcoin data available, 2) identifies emerging trends within the Bitcoin market, 3) uncovers new insights from visual analytics which are much more engaging and comprehensive, and 4) identifies patterns and relationships from complicated data. There are many other similar websites like Coin Dance [154], and Blockstream [155]. This category has the second highest number of projects in the ecosystem and 387 unique developers. Moreover, majority of the projects are developed in CSS, JavaScript, and HTML. The most active projects in this category is OpenBazaar Desktop [156], it is an interface for OpenBazaar Bitcoin node.

Node: A node is a device, usually a computer or any other electronic device, that participates in running a cryptocurrency network. Casa [157] is an example of an application that is used to run a node of the Bitcoin network. Bitcoin Lightning [158 is another example of a Bitcoin node. This category has 26 projects and 421 unique contributors. One of the most active projects in this category are Eclair 159

Wallet: Wallets are discussed in detail in Section 2.3.2. This class contains all the projects specific to the implementation of wallets. This category includes 51 repos and 291 contributors. The two most popular projects in this category are Electrum [160 and Bither [161]. The top three languages in this category are Java, Python and Ruby.

Mining: Mining is the process of adding various cryptocurrency transactions and evidence of mining work to the blockchain ledger. This category includes repositories that implement features specific to the mining process. There are many software applications for mining Bitcoins, for example, Bitcoin miner [162]. This is a desktop application that can be used as a solo-miner or connect you to a miner pool. BTCMiner [162] is another example of Bitcoin mining software. This category has 
26 projects and includes 36 contributors. 0xbitcoin $[163]$ is one the projects in this category. The programming languages in this category are Groff and shell.

Trade: Trading Bitcoins means selling or buying coins. There are multiple platforms through which we can trade Bitcoin. MetaTrader5 164 is a popular multifunctional platform offering state-of-the-art trading capabilities, technical analysis tools, and advanced auto trading systems. This category includes 86 projects and 180 contributors, the top two used programming languages are Java and Elixir. The most popular project in this category is Zenbot [165], a command-line cryptocurrency trading bot using Node.js and MongoDB.

Data analysis: The projects that are related to the analysis and prediction on Bitcoin data. For example, a project 166 in this category implements an application that could predict Bitcoin market prices based on the discussions on Twitter. This category covers 25 projects and has 80 contributors.

Payment: Many big businesses are adopting cryptocurrencies as a method of payment. BTCPay [167] is an example of payment software, which also has a public repository in GitHub. Its server is an open-source payment processor. It enables online buyers to accept Bitcoins as a form of payment. There are 29 projects in this category which offer different solutions to the payment systems. HTML is used by majority of the projects followed by Javascript and CSS.

\subsubsection{Clustering Results}

According to the elbow method in Figure 3(a), we choose $K=4$. Figure 3(b) shows four clusters returned by $\mathrm{K}$-means with $\mathrm{K}=4$. Cluster3, Cluster0, Cluster2, and Cluster1 has 25, 20, 375 and 15 data points, respectively.

Cluster evaluation: Figure 4(a) shows the Silhouette score for different segmentings of the dataset. For $\mathrm{K}=4$, the Silhouette score is 0.619 .

Now that we have segmented our projects, it would be nice if we would know 


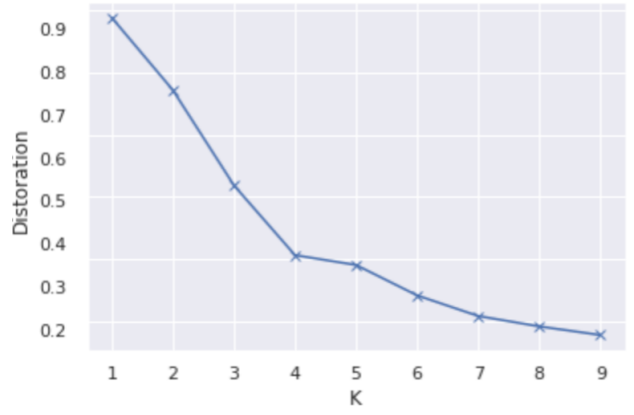

(a) Elbow method

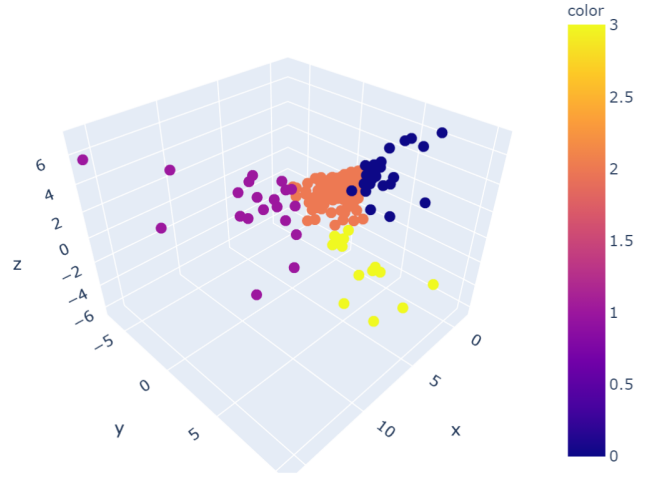

(b) Clusters

Figure 3: Clustering results.

what makes each cluster unique. This will help us better understand which types of data-driven clusters we have. To do so, one simple approach is to plot every variable in each cluster to see the differences between the clusters. We have more than ten features, thus this method fails. Since it would be difficult to compare the variance in all the features across clusters; for simplicity, we select eight most important features and compare them across the clusters. Thus, we use the variance between averaged groups. One assumption of variable importance in cluster tasks is that if the average value of a variable ordered by clusters differs significantly among each other, that variable is likely important in creating the clusters. We start by simply aggregating the data based on the generated clusters and retrieving the mean value per variable. Next, we simply calculate the variance of means between clusters within each variable and select the top 8 variables with the highest variance. From Figure 4 we can can see the difference between the clusters. The number of tokens vary the most between the clusters, for example, Cluster2 have the lowest number of token count, while Cluster 3 has the highest number of tokens, and there a significant difference between these two clusters in terms of token count and code complexity. The least important variable is the number of authors of the projects, which does not change significantly across clusters. Cluster 1 has the oldest projects followed by Cluster3. 

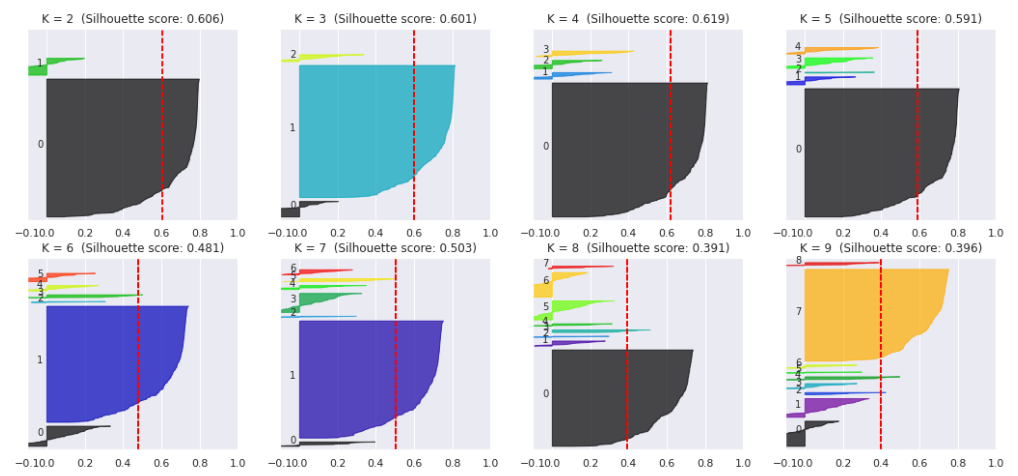

(a) Silhouette Analysis

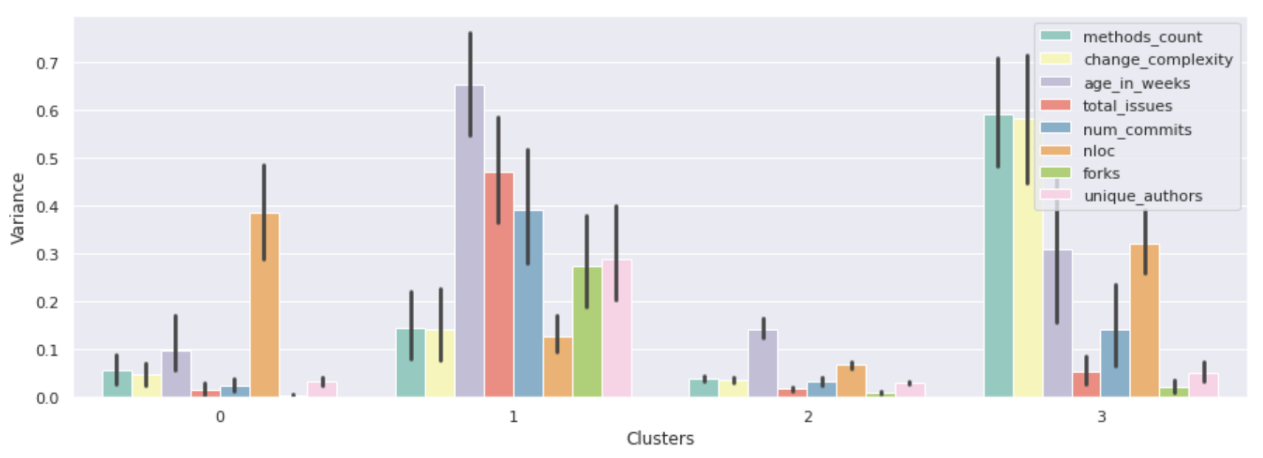

(b) Variance Within Variables and Between Clusters

Figure 4: Cluster evaluation.

Analysis of each cluster: Cluster0 consists of the projects that have the lowest average number of commits, the lowest average number of watchers, and are the second youngest in age. Furthermore, Cluster0 consists of the projects with the highest number of lines of code on average per repository as well as the highest amount of lines added and the second highest amount of lines removed, as well as having the second lowest change in complexity. The characteristics of Cluster0 suggest that it consists of new projects with a small number of active contributors and that it is too early in the project life to determine whether it would be popular or not.

Cluster1 consists of the projects that have the highest average number of commits, as well as the highest average number of watchers, unique authors, forks and 
average age in weeks. Cluster 1 also consists of the projects with the second lowest number of lines of code, the lowest number of lines added, the second lowest number of lines removed, and the second highest change in commits. The characteristics of Cluster1 suggests that it consists of the oldest and most popular repositories that are still viewed but are not changing as much as the younger repositories which is reinforced by the fact that cluster consists of repositories with a higher average change in complexity than Cluster1.

Cluster2 consists of the repositories that have the second lowest number of commits, average number of watchers, average number of forks and average age in weeks and the lowest average number of unique authors and the lowest average change in complexity. Cluster2 also contains repositories with the lowest number of lines of code, the second lowest number of lines added, and the lowest average lines removed. The characteristics of Cluster2 suggests that it represents projects that have failed to gain popularity.

Cluster 3 consists of the projects with the second highest average number of commits, average number of watchers, unique authors, forks, age in weeks. As well as the second highest average number of lines of code and the second highest average lines added and removed. Cluster3 also consists of repositories with the highest average change in complexity. The characteristics of Cluster3 suggests that it represents projects that are more mature and popular but not as mature and popular as projects in Cluster1. However, the fact that Cluster3 has a higher average change in complexity as compared to Cluster1, this suggests that projects in Cluster3 may be in active development stage until they reach maturity and a reasonable complexity of the projects in Cluster1.

Overall, the four clusters sort themselves neatly into the four following groups: Cluster0 consists of the projects that are young and currently lacking popularity, 
Cluster 1 consists of projects that are mature and the most popular, Cluster 2 represents projects that are more mature but have failed to gain popularity, and Cluster3 is made of projects that are popular and mature but have not reached the popularity of Cluster1 due to the fact that these projects' codebases are quite complex, i.e., have the highest average change in complexity.

Cluster 2 represents the largest portion of the projects, i.e., 374 out of 434 repositories (86.1\%). Therefore, we can conclude that over $85 \%$ of the Bitcoin software ecosystem in GitHub includes relatively young projects (average age is 37.89 weeks) that are still gaining popularity or increasing their code complexity.

Cluster3 contains $3.4 \%$ of the projects within the Bitcoin ecosystem (15 out 434 repositories). Thus, we can make a claim that around $3 \%$ of projects have gained a reasonable level of popularity but are not anywhere near as popular as those of Cluster 1 with an average number of watchers at 57 compared to Cluster1's average of 912 watchers. Cluster 1 includes 20 out of 434 of the projects (4.6\%) and consists of popular and complex code repositories with the highest average number of watchers and unique authors. Finally, Cluster 0 consists of 25 out of the 434 total repositories $(5.7 \%)$ which include young repositories with an average age of 27 weeks that have not been around long enough to determine their success in popularity.

Semi-supervised: Semi-supervised clustering gives a Silhouette score of -0.300 . The best value for Silhouette score is 1 and the worst value is -1 . Values near 0 indicate overlapping clusters. Negative values generally indicate that a sample has been assigned to the wrong cluster, as a different cluster is more similar. Random adjusted score which calculates the known labels for the $80 \%$ of the dataset is -0.030 . Random adjusted score is used to see the similarity between two clusters; it is near to 0 if the labels are assigned randomly, and 1 if the clusters are identical. Furthermore, 23 out 384 repositories are clustered into the right clusters according to the pre-defined categories. Figure 5 shows nine clusters. We can see that clusters are overlapping. 


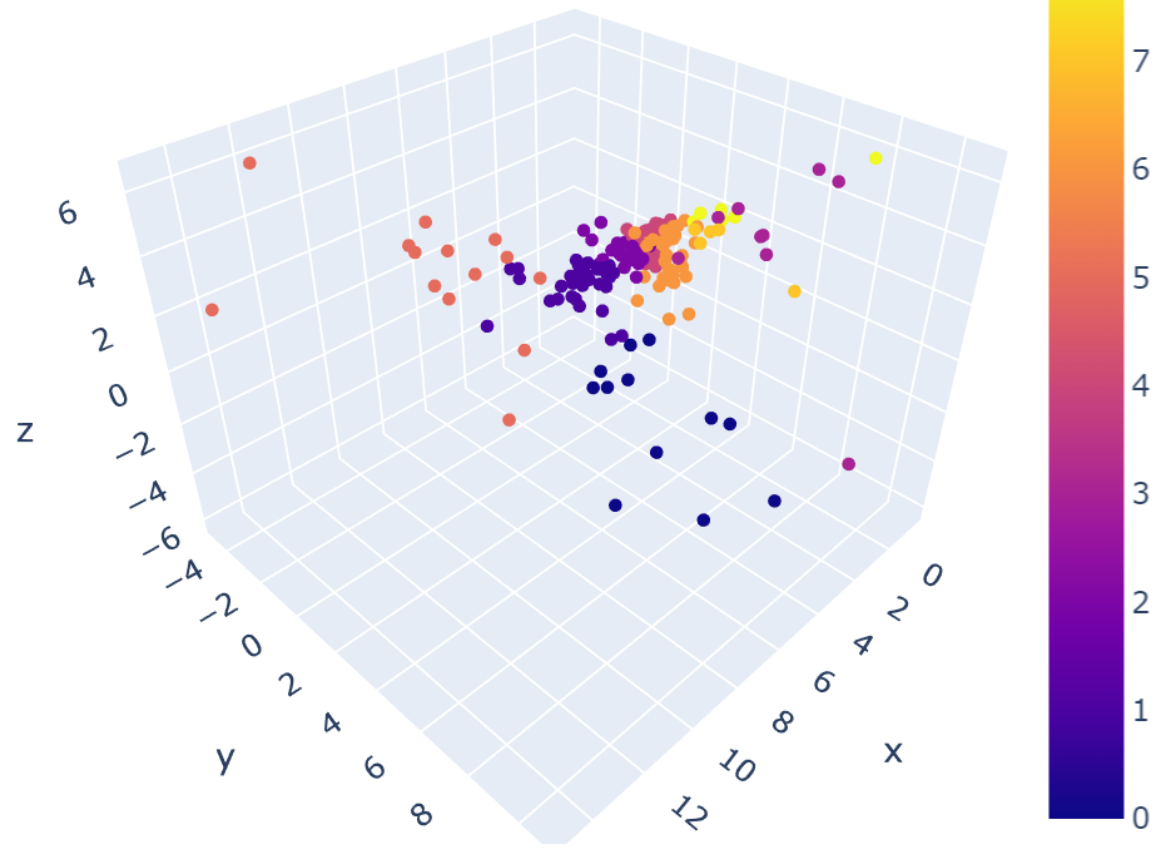

Figure 5: Semi-supervised clustering results.

\subsubsection{Category Prediction}

In this section, we present the results from the decision tree classification for predicting the category of a project. The model achieved an accuracy of $93.156 \%$ and $84.3 \%$ for training set and test set, respectively. Furthermore, the average F1-score is 0.828 as shown in the classification results in Table 12. Recall for class "Data analysis", "Mining", "Node" and "Documentation" is 1, which means that this model can correctly identify all samples of these classes. The precision for "Documentation" is 1 which means that all the positive predicted observations are correctly identified. For class "Development", the model has the lowest recall, which means that this model can identify only $2 \%$ samples of this class. This is the largest category in out dataset. Overall, F1-score is the highest for class "Mining" and "Documentation". If we look at the average values, we observe no significant difference between the 
Table 12: CART classification report.

\begin{tabular}{l|cccc}
\hline & Precision & Recall & F1-score & Support \\
\hline Development & 0.552 & 0.258 & 0.352 & 62 \\
Data analysis & 0.812 & 1.000 & 0.896 & 56 \\
Payment & 0.951 & 0.935 & 0.943 & 62 \\
Services & 0.729 & 0.729 & 0.729 & 48 \\
Mining & 0.965 & 1.000 & 0.982 & 55 \\
Trade & 0.780 & 0.807 & 0.793 & 57 \\
Node & 0.884 & 1.000 & 0.938 & 61 \\
Wallet & 0.750 & 0.894 & 0.816 & 47 \\
Documentation & 1.000 & 1.000 & 1.000 & 54 \\
Accuracy & & & 0.843 & 502 \\
Macro average & 0.825 & 0.847 & 0.828 & 502 \\
Weighted average & 0.825 & 0.843 & 0.825 & 502 \\
\hline
\end{tabular}

different metrics.

The micro average AUC score is 0.62 , which means that this model is $62 \%$ correct in distinguishing between different classes. The AUC score for class "Data analysis" and "Payment" is 1 and 0.99 , respectively, while it is the lowest for class "Trade" with value of 0.33 .

Answer to RQ1: Based on our findings, the Bitcoin software ecosystem in GitHub is represented by 531 projects as of 01-04-2018. The manual classification of these projects resulted in nine categories, including wallets, services, trade, node, mining, development, payments, documentation, and data analysis. On the other hand, unsupervised clustering resulted in four major clusters of the data. Semi-supervised clustering offered least promising outcomes for our dataset, while supervised classification achieved an average F1-score of $84 \%$ when predicting project category. 
Table 13: Descriptive statistics for health categories.

\begin{tabular}{l|c|c|c|c|c|c|c|c|c}
\hline Feature & \multicolumn{3}{|c|}{ Healthy } & \multicolumn{3}{c|}{ Low Risk } & \multicolumn{3}{c}{ At Risk } \\
\hline \# of projects & \multicolumn{3}{|c|}{85} & \multicolumn{3}{c}{322} & \multicolumn{3}{c}{74} \\
\hline & Min & Mean & Max & Min & Mean & Max & Min & Mean & Max \\
\hline Commits & 12 & 752.188 & 5,049 & 10 & 676.618 & 10,901 & 10 & 246.918 & 9,580 \\
\hline Contributors & 2 & 9.141 & 91 & 2 & 6.888 & 442 & 2 & 2.189 & 7 \\
\hline Issues & 2 & 202.352 & 2,432 & 0 & 112.357 & 13,038 & 0 & 1 & 12 \\
\hline Watchers & 3 & 355.964 & 7,853 & 0 & 239.034 & 32,056 & 0 & 0.918 & 7 \\
\hline Forks & 0 & 134.529 & 2,212 & 0 & 88.267 & 1,4951 & 0 & 0.554 & 6 \\
\hline Age & 16.285 & 127.304 & 343.571 & 0.857 & 41.215 & 380.0 & 0.428 & 10.050 & 15.714 \\
\hline CC & 0 & 10.610 & 29.901 & 0 & 33.040 & 984.496 & 4.544 & 69.718 & $1,929.768$ \\
\hline
\end{tabular}

\subsection{RQ2.1: How Healthy is the Bitcoin Ecosystem in GitHub?}

In this work, the Bitcoin software ecosystem in GitHub is classified into three classes of health - "Healthy", "Low Risk", and "At Risk" with 85, 322 and 74 projects, respectively. Table 13 shows the min, max and mean values for the features we used to classify the projects.

The average number of commits in class "Healthy" is 752.188 , while it is 676.618 in class "Low Risk", and 246.918 in class "At Risk". Similarly, the average number of contributors in class "Healthy" is 9.141, 6.888 in "Low Risk", and 2.189 in class "At Risk". Whereas the average number of CC is 10.610 in class "Healthy", 29.901 in class "Low Risk", and 69.718 in class "At Risk". "Healthy" projects appear to be mature, very active projects (on average around 750,000 commits), with large community of contributors, high popularity, high number of forks (forks are used to experiment with changes without affecting the original project), high number of issues (issues are representing requests to fix bugs or add new functionality), and low code complexity. "At Risk" projects can be defined as 2-3 month old repositories 
with a few or zero watchers, forks, issues, much smaller community of contributors, and of high code complexity. The "Low Risk" projects are performing well on at least one category of health. For example, this class includes a project which has the maximum number of contributors in the ecosystem, i.e., 442, but the project also has the highest cyclomatic complexity which makes it at "Low Risk" and not "Healthy".

Answer to RQ2.1: Majority of the projects of the Bitcoin ecosystem demonstrate "Low Risk" health indicators which is a good sign for the overall health of the ecosystem, while $17 \%$ of projects are appear to be "Healthy" and only $15 \%$ of projects are deemed to be "At Risk".

\subsection{RQ2.2: What Are the Trends in the Health of the Bitcoin Ecosystem?}

In this section, we present the results of our analysis of the ecosystem's health trends. We analyze the trends over nine categories which have been identified in Section 4.2.1.

\subsubsection{Activity}

Table 14 shows the number of projects created each year. Five projects that were created in the year 2010, when Bitcoin has emerged as a cryptocurrency, are still being actively developed. In 2011, 14 more projects have been created, but in 2012 only 4 new Bitcoin related projects were added to GitHub. The number of new projects is increasing after year 2013. In 2014, 30 new projects have been created, and in 2017 the number of new projects has jumped to 218. 2018 is marked with 83 new projects created just in the first 4 months of the year.

Contributions: The average number of commits per year per project is shown in Figure 6. We can observe that the trend is fluctuating through the years. 
Table 14: Number of projects created per year.

\begin{tabular}{l|lllllllll}
\hline Year & 2010 & 2011 & 2012 & 2013 & 2014 & 2015 & 2016 & 2017 & 2018 \\
\hline Repos & 5 & 14 & 4 & 12 & 30 & 31 & 23 & 218 & 154 \\
\hline
\end{tabular}

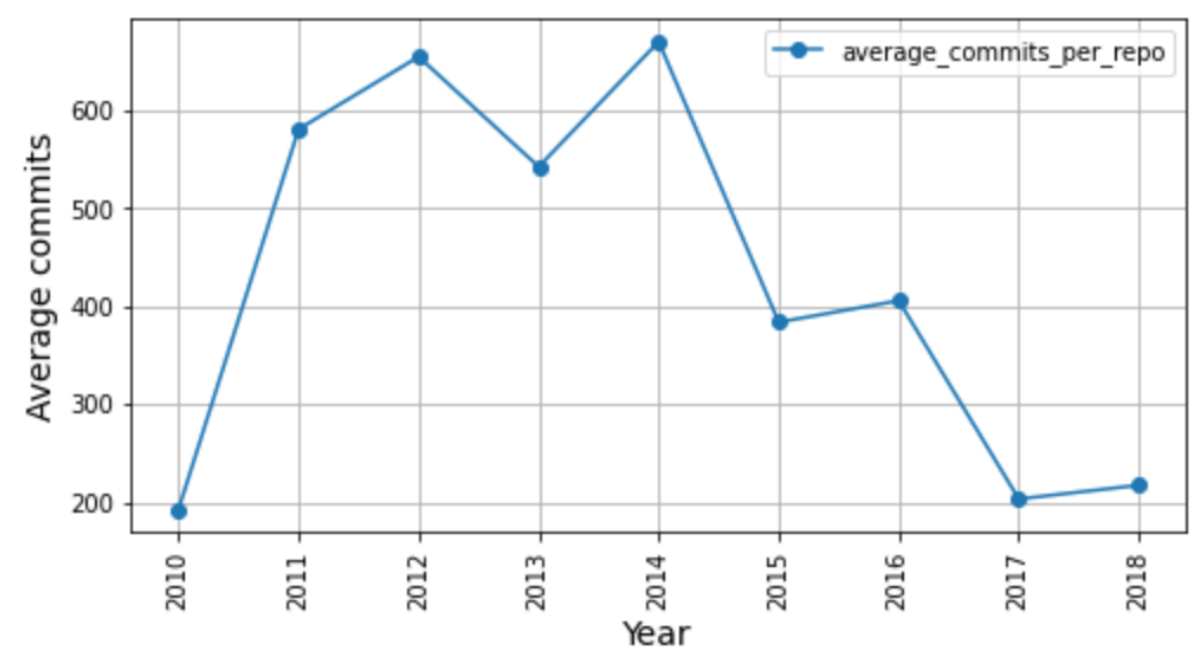

Figure 6: Average number of commit per year per project.

There is a significant increase from year 2010 to 2011, but the rest of years it is not changing in one direction. Table 14 shows the number of projects created each year. As we can see from the table the number of projects is increasing rapidly after year 2016. We can observe an increased interest in the Bitcoin related development and representation of projects in GitHub since 2017.

Figure 7 shows the year-to-year growth rate in terms of commits. 


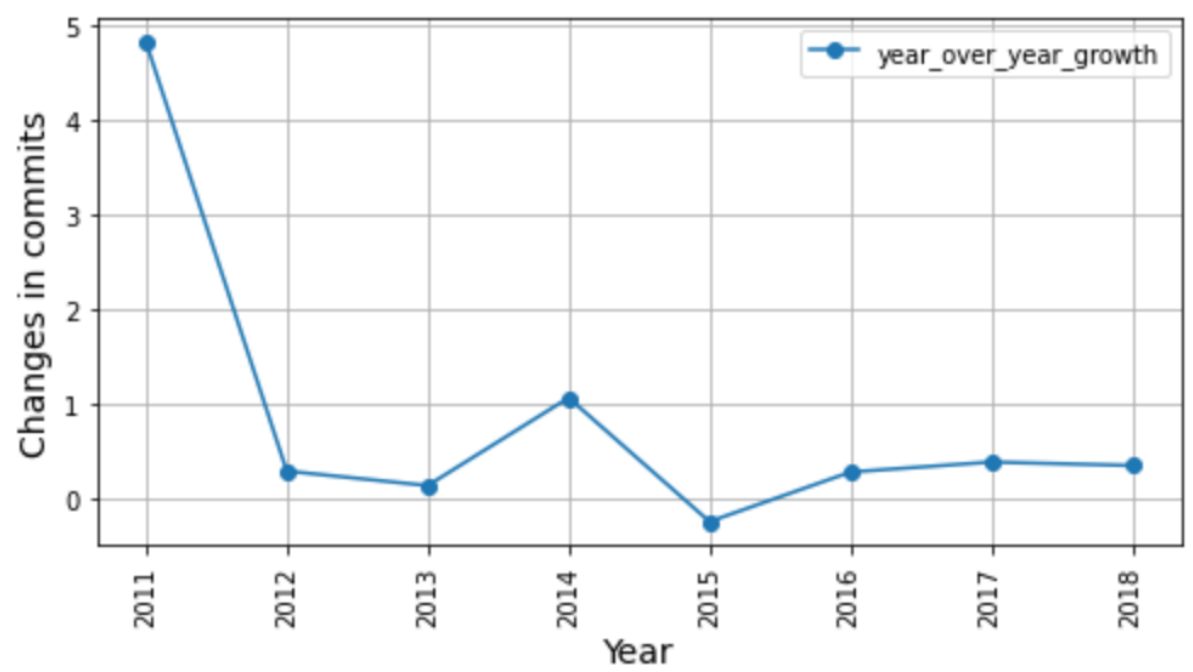

Figure 7: Change in the number of commits per year.

The growth rate is decreasing till year 2015 except 2014, where there is a slight increase and the this change is significant from year 2011 to 2012. After 2015 the growth rate is constantly increasing till year 2018. Furthermore, Figure 8 shows the number of commits per each category in the ecosystem.

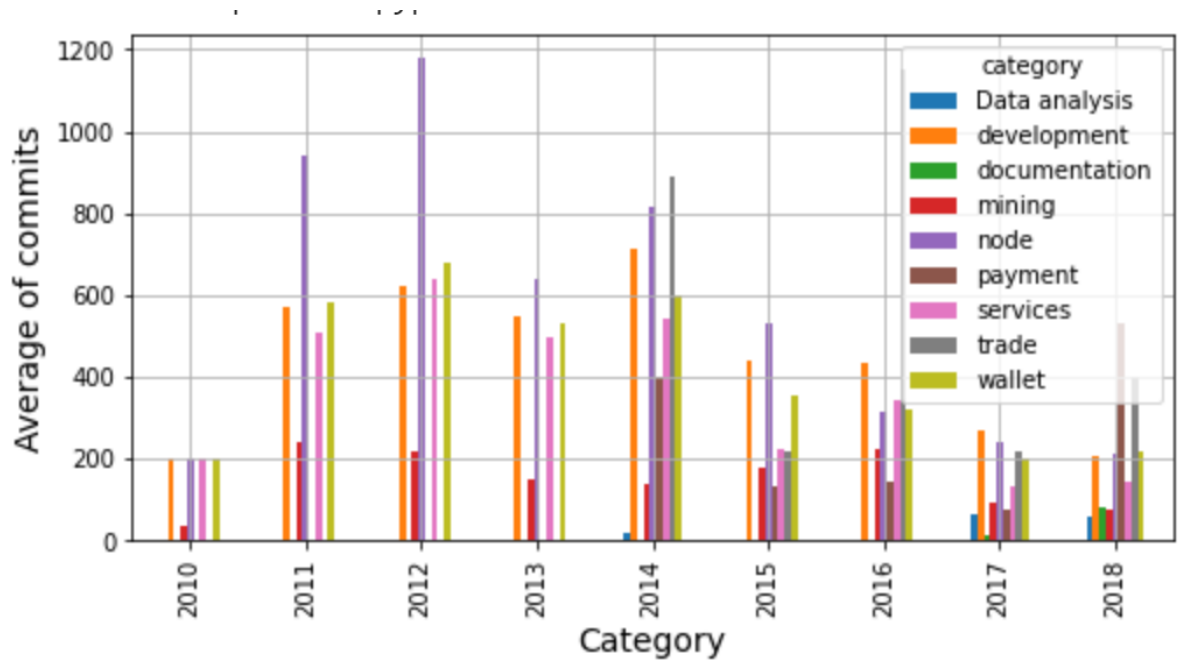

Figure 8: The average number of commits per category and per year.

Till year 2014, the Bitcoin ecosystem was represented only via five categories such as development, mining, node, services, and wallet. In 2010, four categories 
(except mining) have equal number of commits, i.e., 200. Up until year 2013, mining category of projects has the lowest number of commits. After 2011, node category has the highest number of commits, while there is no significant difference between other four categories. In 2014, documentation, trade, and payment categories have emerged. Documentation has the lowest number of commits, while trade has the highest number of commits followed by node. The documentation category shows no activity in 2015 and 2016, but shows slight activity in 2017 and 2018. In 2017, data analysis has emerged as a category with the second lowest activity. In 2018, payment has the highest number of commits, followed by trade, while development, node, and wallet have almost the same number of commits.

Figure 9 demonstrates the average number of commits per developer. The average is taken by dividing the total number of commits by the number of projects a developer has contributed to. The mean number of commits per developer is shown by the green line near to the maximum values in the plot. The number of commits for the majority of the developers is below the average, i.e., 35 commits.

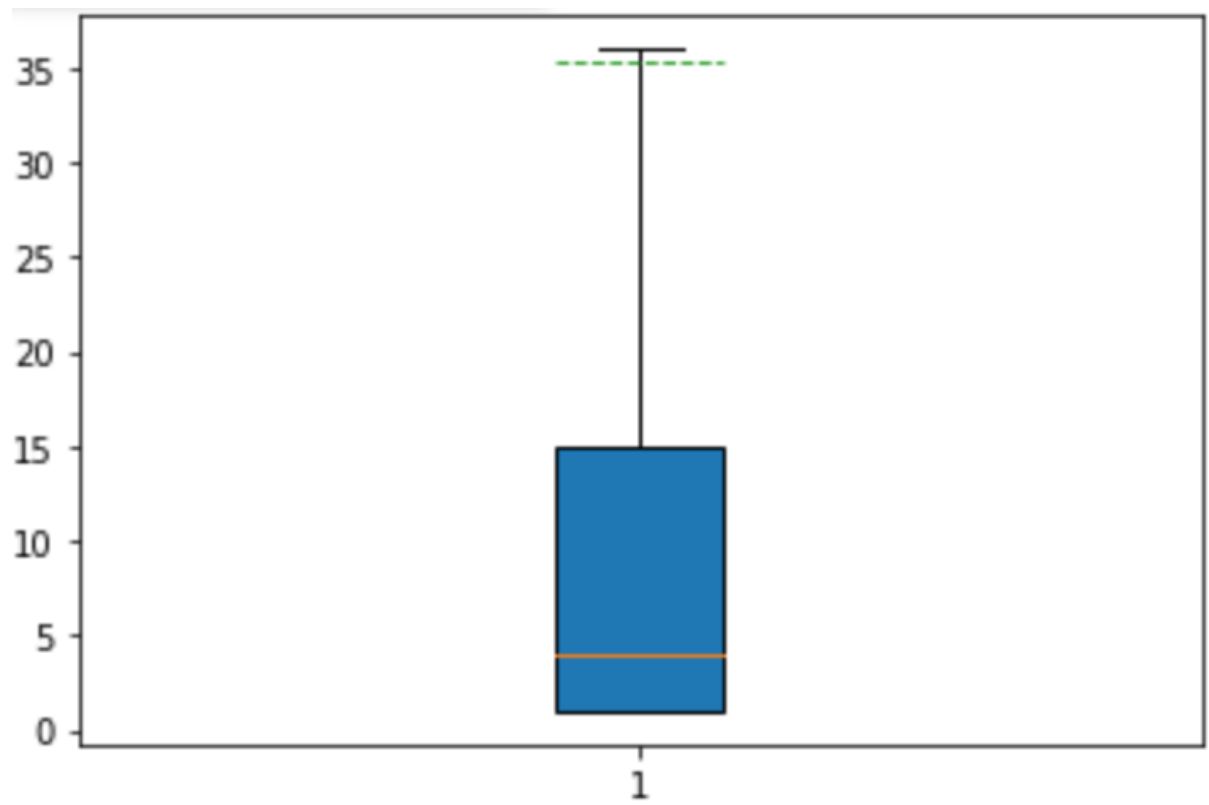

Figure 9: Average number of commits per developer. 
Collaboration of authors: Involvement of different developers is measured in terms of their input to various projects is studied in this section. We calculate the following metrics in order to evaluate the contributions of authors to the Bitcoin ecosystem.

1. The average number of developers committing to the ecosystem each year,

2. Loss of contributors,

3. Developers retention rate in the ecosystem, and

4. Number of developers contributing across different projects and across different categories.

Figure 10 shows the average number of authors per year per projects. As shown in the Figure 10 there is a surge in the number of developers contributing to the ecosystem in year 2011, but this number decreases in the following years.

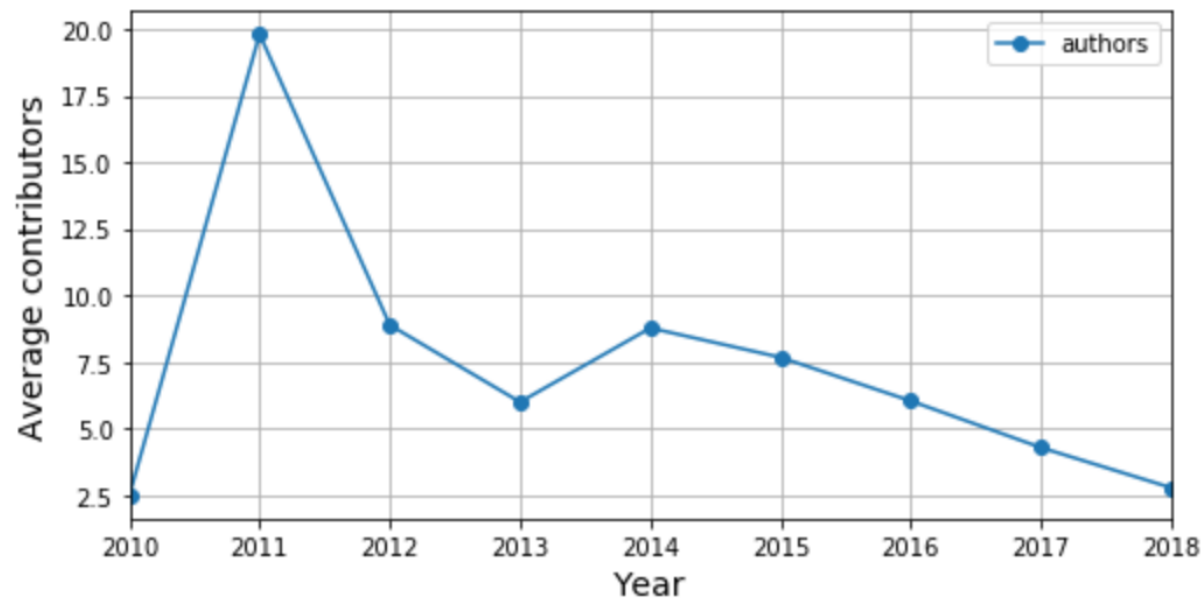

Figure 10: Average number of authors per year per project.

To calculate the loss of contributors to the ecosystem we counted the number of authors with their last commit for each year. Figure 11 shows the loss of contributors within the ecosystem, i.e., the number of developers who contributed to a project in 
a certain year but have not contributed again in the following years. As indicated in the figure the loss in contributors is gradually increasing throughout the years. In 2010, there were only 2 developers who haven't committed to the Bitcoin ecosystem again. While in 2016, there are 111 authors who haven't contributed in year 2017 and the first quarter of 2018.

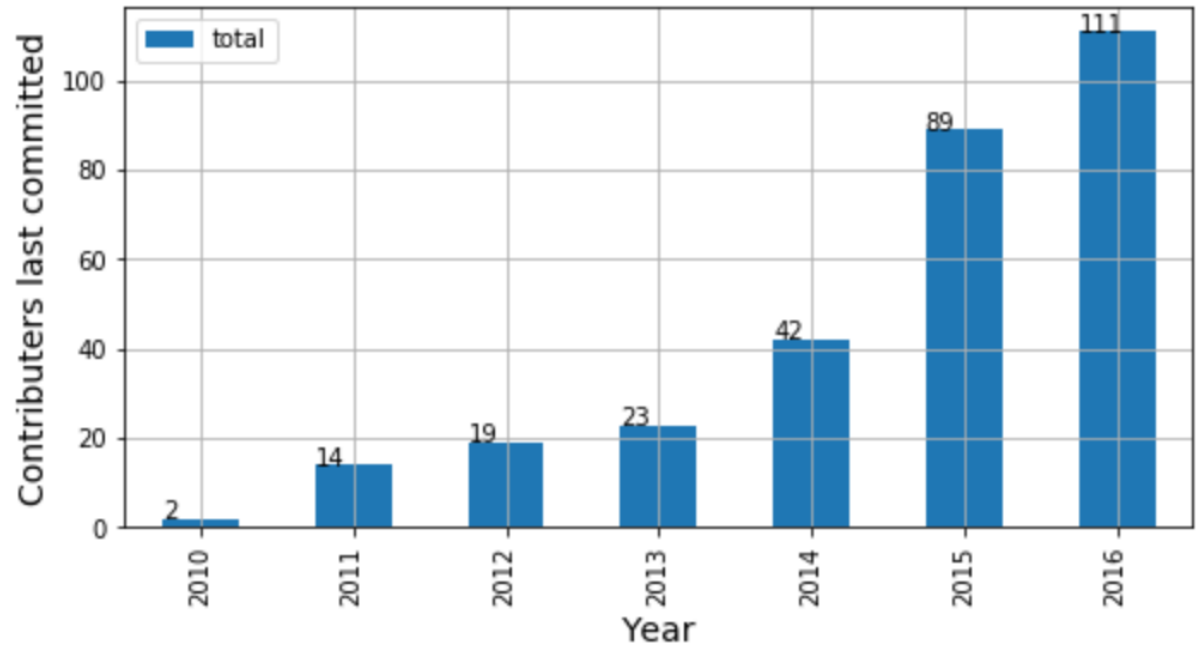

Figure 11: Loss of contributors per year.

To calculate the retention rate of developers, we subtracted the number of newcomers from the number of authors making their last commit as shown in Figure 12 , This gives us an insight about how many developers are being retained throughout the years. 


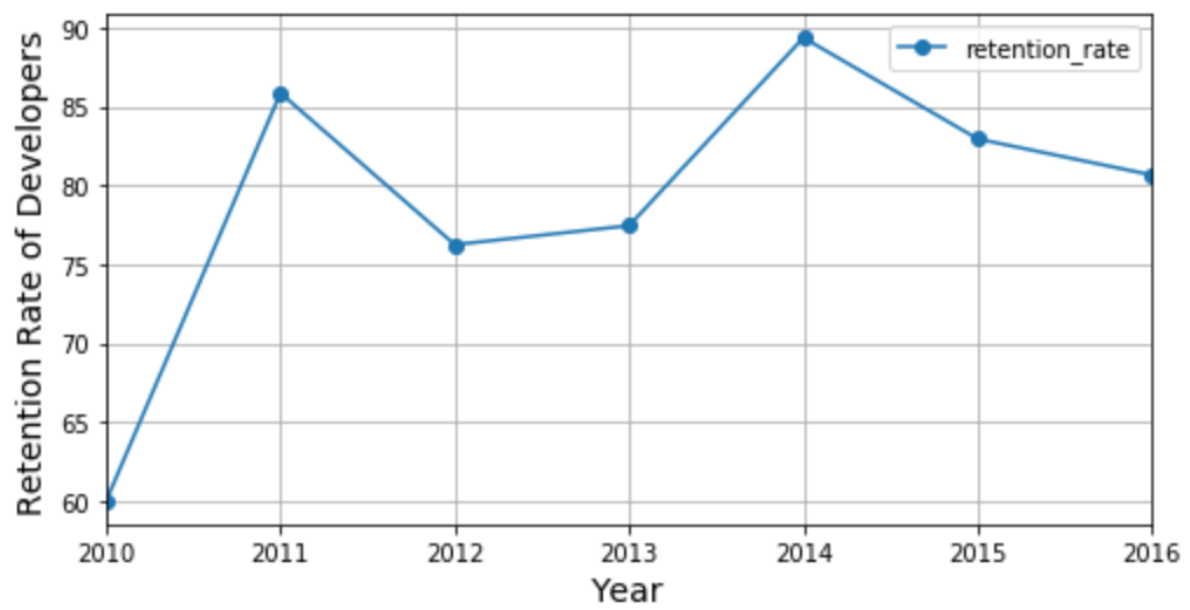

Figure 12: Developer retention rate over the years.

$60 \%$ of the developers who contributed in 2010 have stayed in the ecosystem, while more than $85 \%$ of the developers who submitted a commit in 2011 continued their participation and contribution to the ecosystem. This number decreases to $76 \%$ in 2012. The retention rate is the highest for 2014 which is almost $90 \%$ of developers. Since 2014 the retention rate has been decreasing.

Finally, we want to see whether developers within the Bitcoin ecosystem are collaborating and interacting with each other. To determine this we have analysed developers who are working on more than one project and whether these projects belong to different categories of the ecosystem. As shown in Figure 13, 88\% of the developers are contributing to only one project, and the remaining $12 \%$ are working on more than one project, with $7 \%$ of developers contributing to 2 projects, $2 \%$ to 3 projects, $1 \%$ to 3 projects and $1 \%$ of developers to 5 and more projects. 


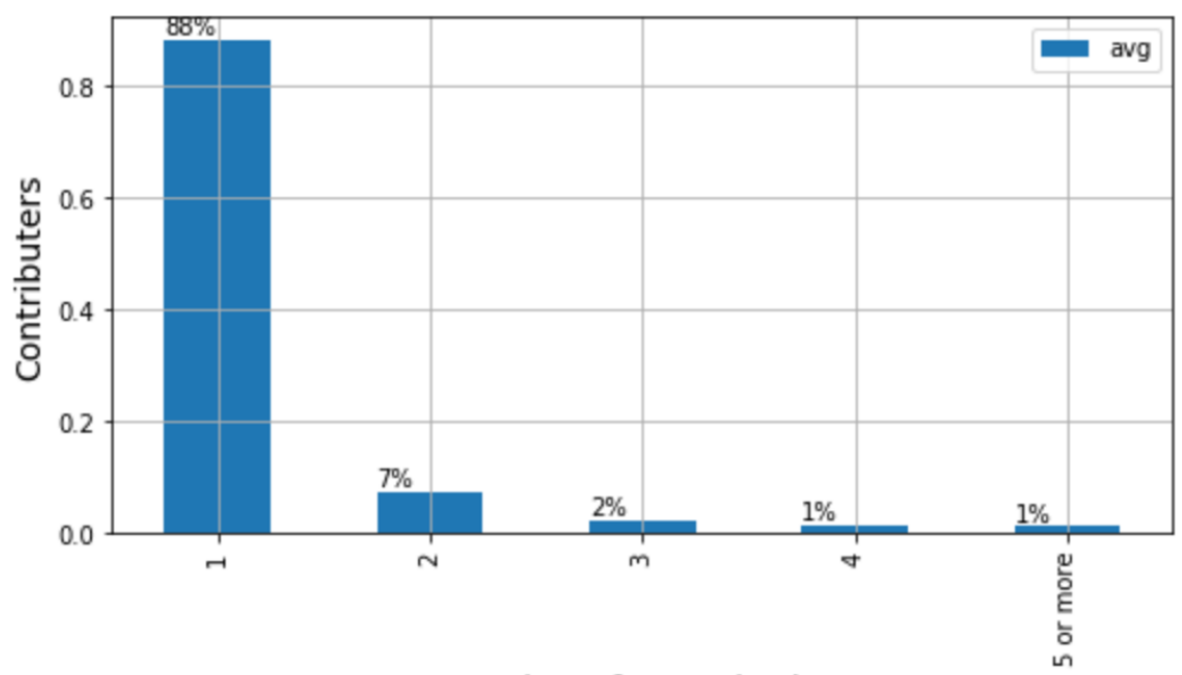

Number of Repositories

Figure 13: Developer collaboration within the ecosystem.

Figure 14 shows the knowledge transfer across different categories in the Bitcoin ecosystem. The Figure 14(a) shows three most collaborating categories where 56 developers are working in both development and services, and 84 developers are working in development and node. 84 is the highest number of developers contributing across different categories, following by 76 developers who are working in development and services. Figure 14(d) shows that projects in wallet, trade and development categories do not have any common contributors.

Issues: The average number of issues created over the years was calculated by dividing the number of issues with the number of projects per year. Figure 15 indicates stability in the average number of issues. As we see, the number of issues is decreasing constantly after year 2011, meaning that projects' code is becoming less bug prone. 


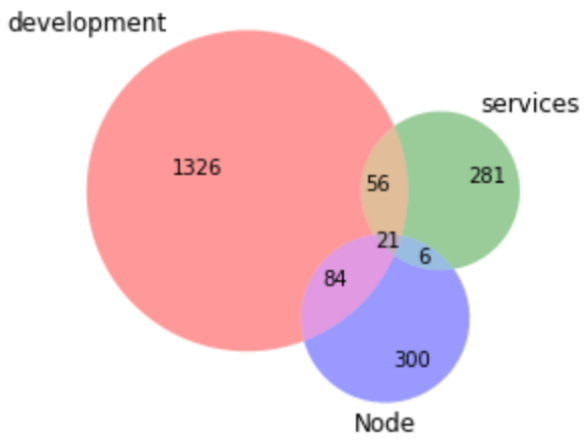

(a) Top collaborating categories

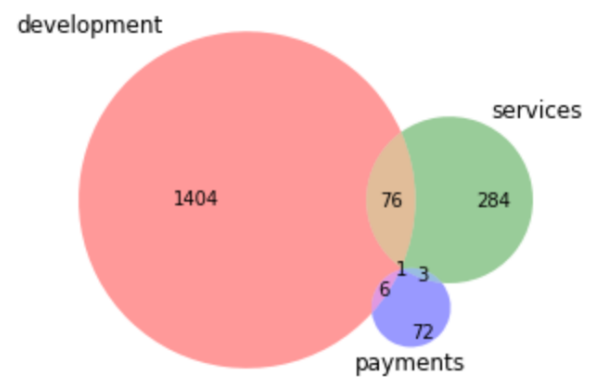

(c) Limited collaboration

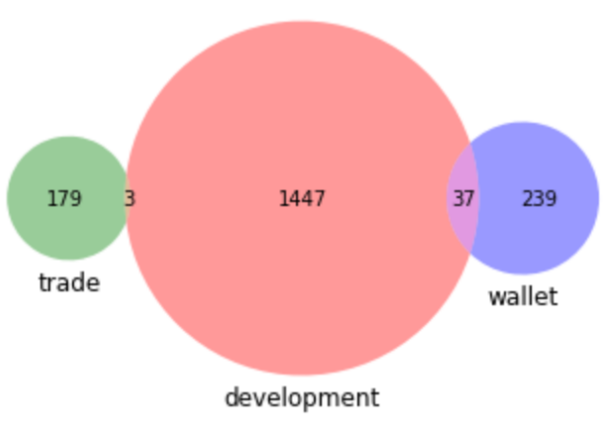

(b) Limited collaboration

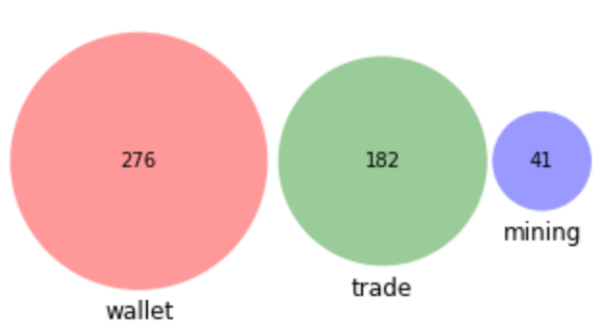

(d) Lack of collaboration

Figure 14: Collaboration across different categories of the ecosystem. 


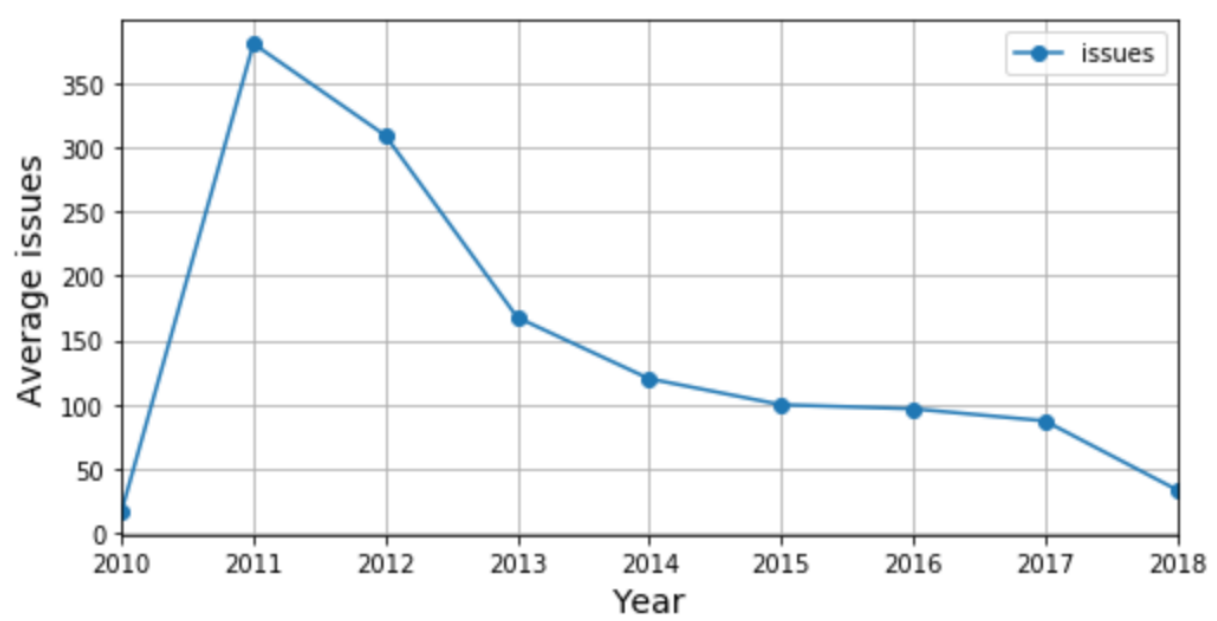

Figure 15: Average number of issues per year per project.

Figure 16 shows the average number of issues within different categories. It shows that development is the dominant category in terms of number issues, followed by wallet from year 2012 to 2014. After 2015, the most of issues were created are in the node category which may appoint to the popularity and higher contribution to this category in the recent years.

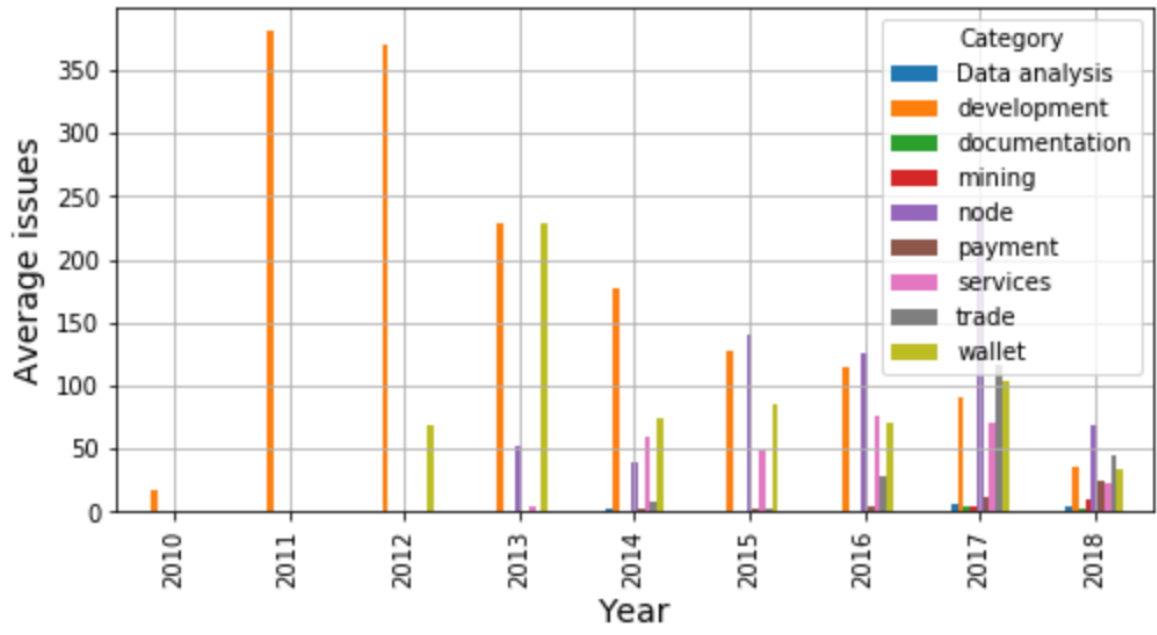

Figure 16: Average number of issues per year per category. 


\subsubsection{Popularity}

To determine the popularity of a project in the ecosystem, we analysed the number

of people starring (watching) a repository and the number of forks. Figure 17 shows the average number of watchers per category throughout the years. Due the fact that we had only two projects in year 2010, the average number of watchers is higher than the normal. After 2011 the number of watchers is decreasing till year 2013. However, after that the number is slightly increasing throughout the rest of years.

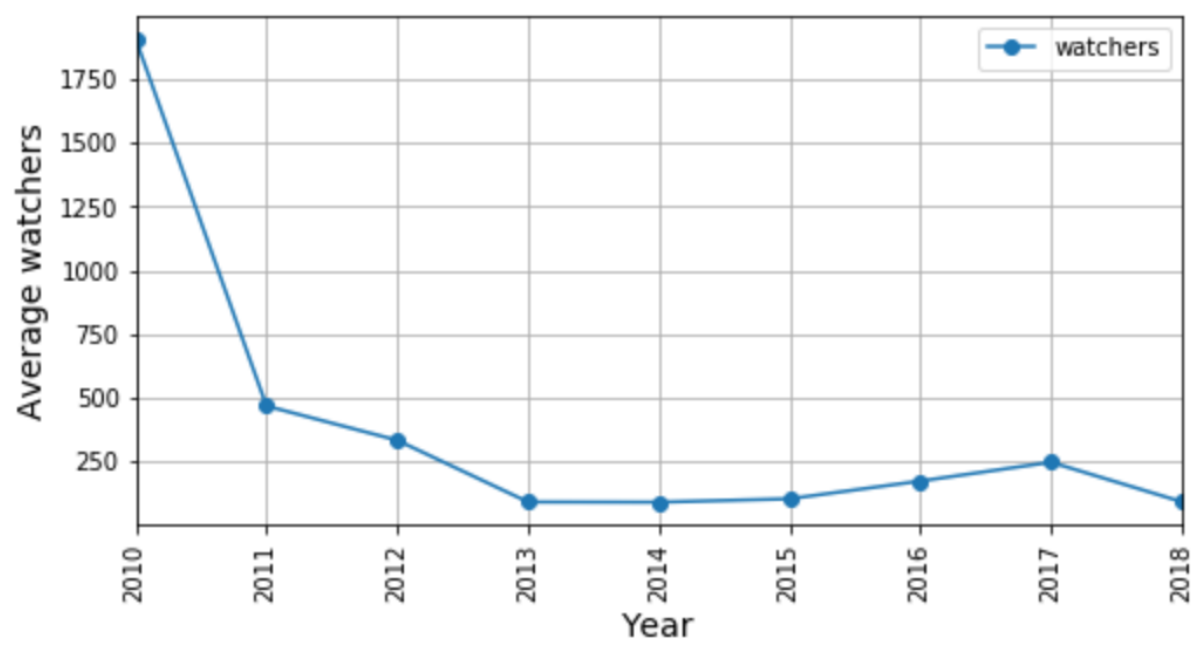

Figure 17: Average number of watchers per year per project.

Figure 18 shows the average number of watchers for different categories throughout the years. In 2010 and 2011, the only category is development. After 2012, wallets projects are gaining attention and it becomes the second popular category till 2014 . In 2015, node is gaining more popularity. Between 2015 and 2016, node stays the second popular category, while in 2017 and 2018 node becomes the most popular category followed by development. 


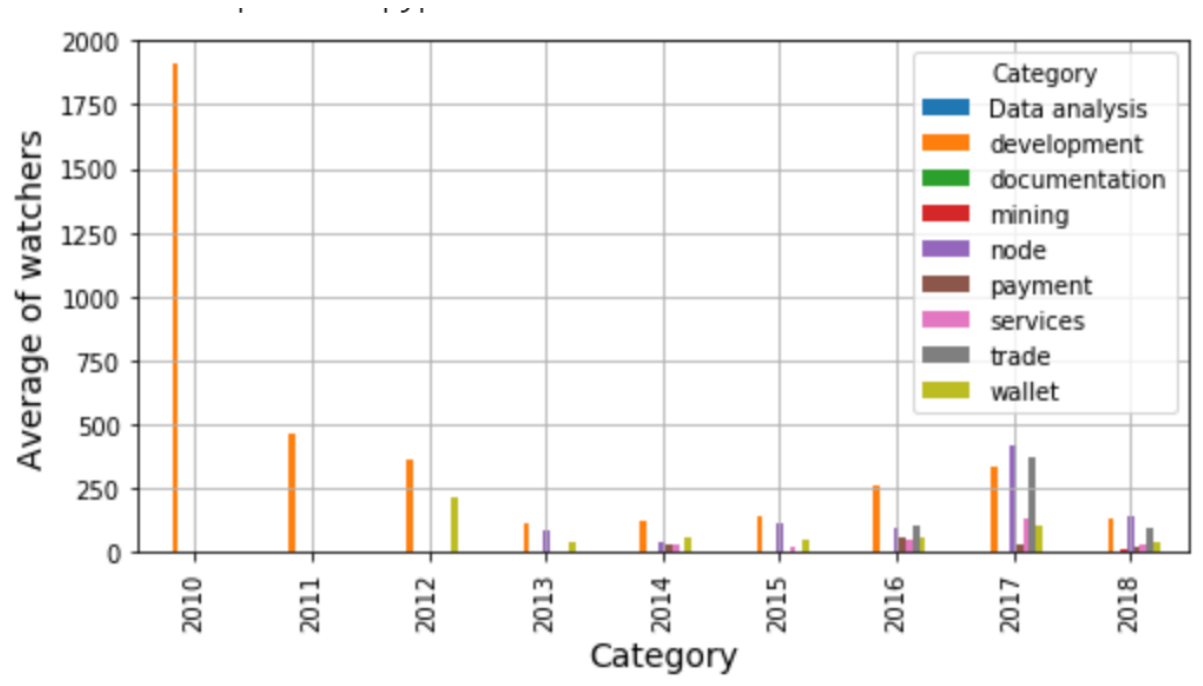

Figure 18: Average number of watchers per year and per category.

Figure 19 shows the average number of forks each year. The number of forks on a project serves as a proxy for the popularity and activity of the project as discussed in Section 4.3.1. Forks indicate the number of projects which are forked from any project in the ecosystem. In 2010, there are 56 forks per project, this number is decreasing till year 2012. In 2015, we have an average of 120 forks which is the highest number throughout the years. This number is significantly dropped to an average of 40 in 2016, but afterwords it is constantly increasing.

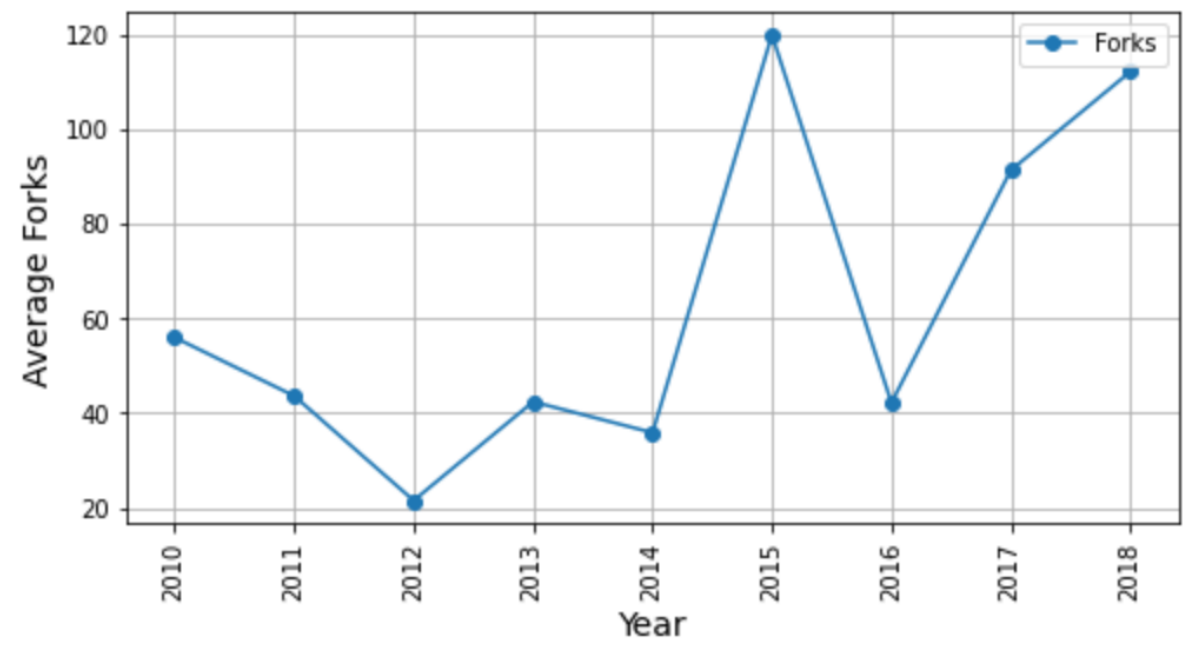

Figure 19: Average number of forks per year. 
Figure 20 shows the number of projects which are forked from a Bitcoin project in each category. As we can see that in 2010 and 2016 all the forks are from the projects in the development category. In 2017, there are some forks from services category, but still development is the leading one. In 2018, the forks are from all the categories, the highest number is for trade followed by node.

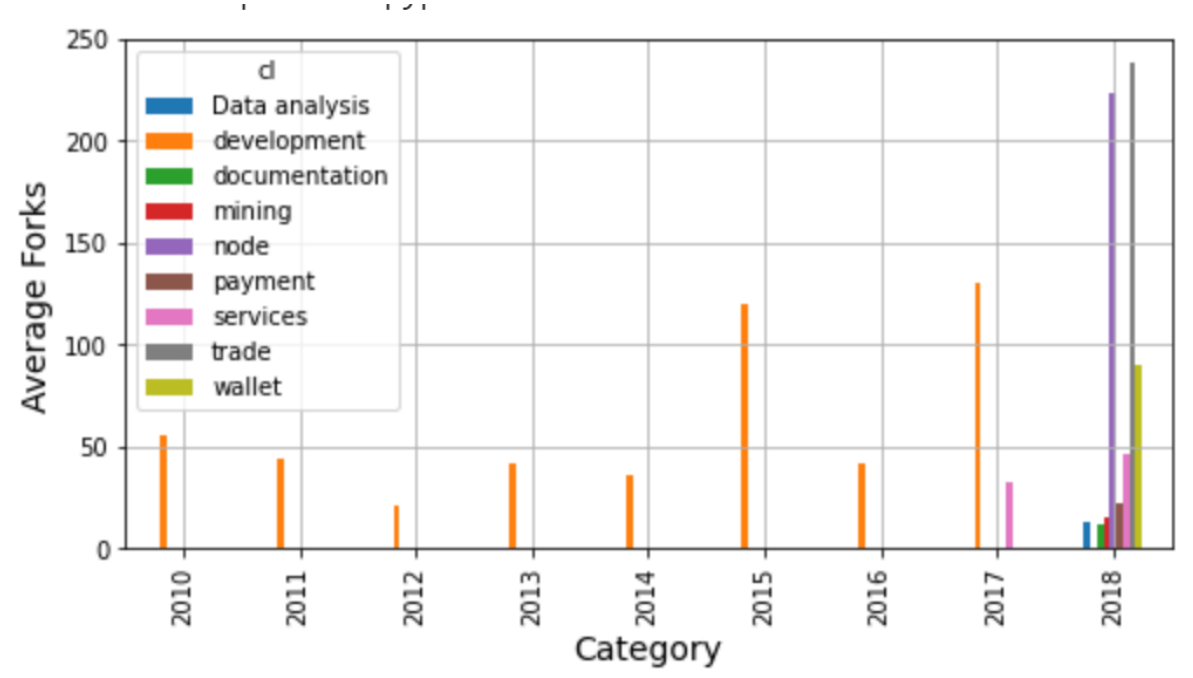

Figure 20: Average number of forks per category.

\subsubsection{Code Quality}

Figure 21 shows the average code complexity per project per year. The higher the complexity the lower the code quality. As discussed in Section 4.3.1, high CC means more independent paths in a module that would require more tests, as well as higher cognitive loads from developers to comprehend and maintain the system.

There is a significant decrease in Cyclomatic Complexity in 2011. From 2011 to 2012 it is increased almost by 100 independent paths through a module. It is decreasing till year 2015, but then it is again increasing till year 2017. Figure 22 shows complexity over the categories. In 2010, all the categories such as development, node, services has the same complexity, while mining has the lowest complexity. From 


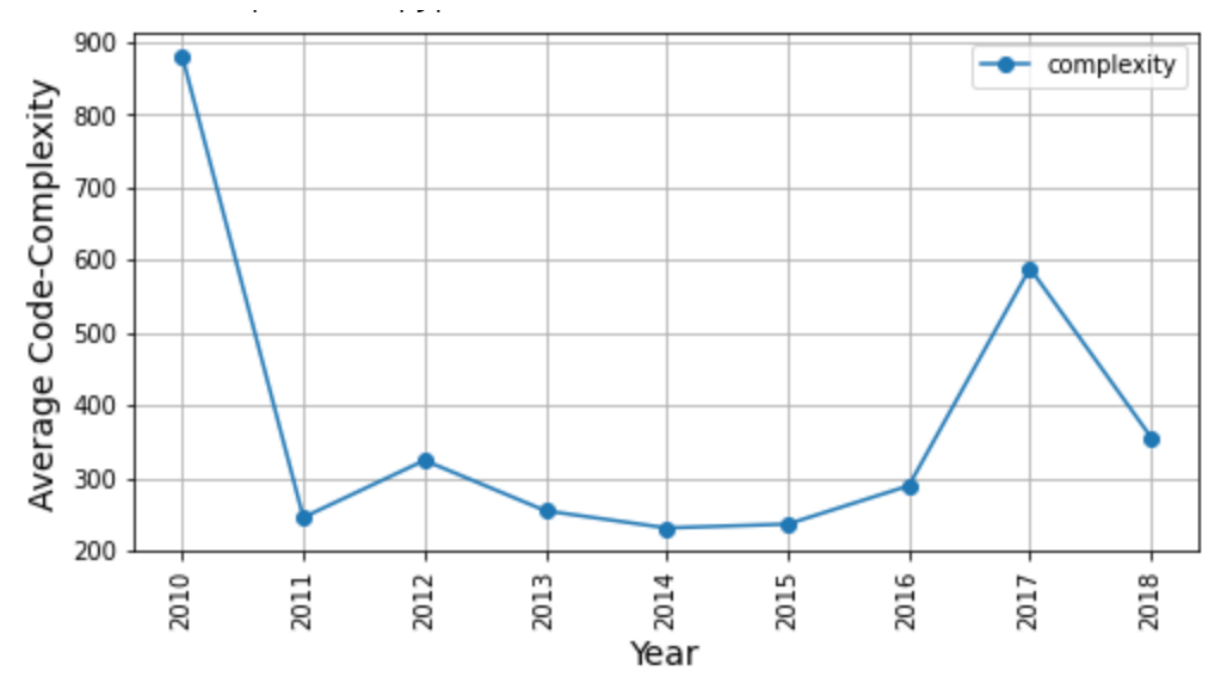

Figure 21: Average code complexity per project.

year 2011 to 2014, node has the highest complexity, while in 2017 it is almost 900 for development and around 200 for the rest of the categories. Mining overall has the lowest complexity less than 200, but in 2018 it reaches the complexity of 1,200 unique paths.

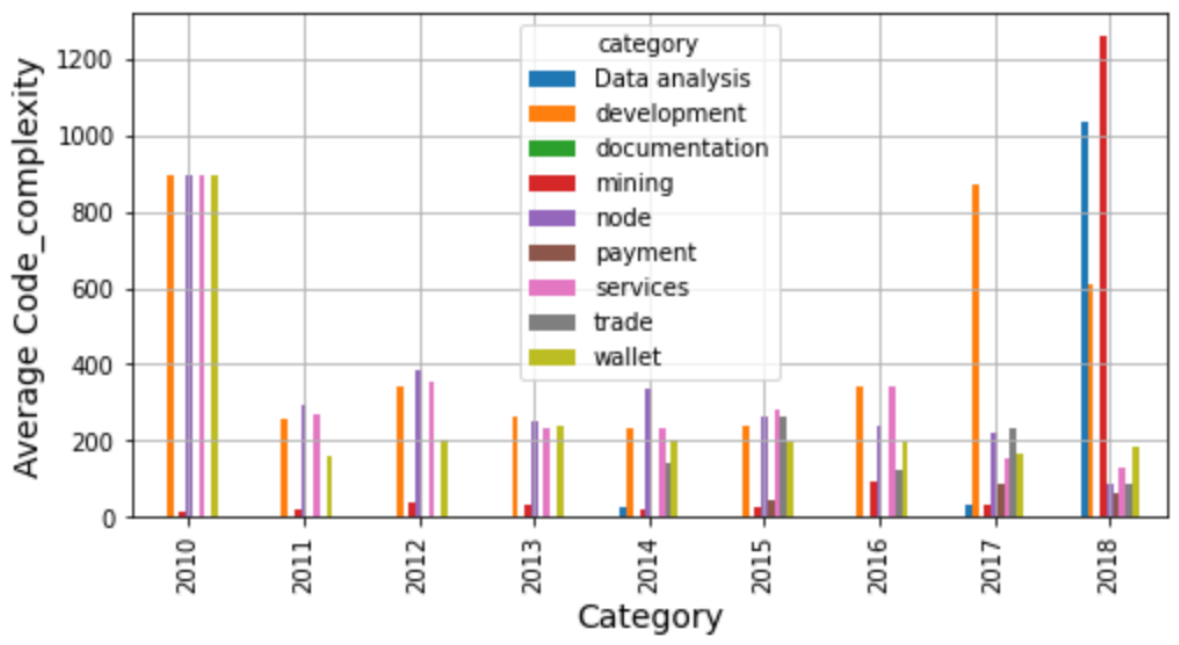

Figure 22: Average code complexity per category. 
Table 15: Descriptive statistics for projects' age.

\begin{tabular}{l|l|l|l|l|l|l}
\hline Mean & Std & Min & $25 \%$ & $50 \%$ & $75 \%$ & Max \\
\hline 51.634 & 74.128 & 0.428 & 10.428 & 17.571 & 44.428 & 380.000 \\
\hline
\end{tabular}

\subsubsection{Age}

Table 15 shows some descriptive statistics about the age (in weeks) of the projects in the ecosystem. The average age of the projects within the Bitcoin ecosystem is 52 weeks (about a year) meaning that most of the projects are relatively young and in early stage of the development. Maximum age is 380 weeks which is around 7 years and 3 months. The Bitcoin's main repository $[150]$ is the oldest project in the ecosystem that is 380 weeks old, and is a part of development category.

Answer to RQ2.2: Overall, the Bitcoin ecosystem's activity in terms of the growth of projects and overall contributions has been on the rise since 2015; while the developer retention rate has been decreasing. However, the average number of contributors per category has been increasing from 2014. While ecosystem's popularity and code complexity is growing every year, cross-category contribution and collaboration between developers has been very low.

\subsection{RQ3: How Accurately Can We Predict the Bitcoin Ecosystem's Health?}

In this section we present the results for each classifier. We will use the models' evaluation methods discussed in Section 4.4.6. We will use decision metrics, classification reports, and ROC to compare the results of four different classification models.

SVM: The total observations in the test set are 242 after a $20-80 \%$ split. SVM achieves accuracy and weighted average F-score of 0.753 and 0.754 , respectively as 
Table 16: SVM classification results.

\begin{tabular}{l|cccc}
\hline & Precision & Recall & F1-score & Support \\
\hline At Risk & 0.745 & 0.953 & 0.837 & 86 \\
Low Risk & 0.646 & 0.545 & 0.592 & 77 \\
Healthy & 0.896 & 0.759 & 0.822 & 79 \\
Accuracy & & & 0.760 & 242 \\
Macro average & 0.762 & 0.753 & 0.750 & 242 \\
Weighted average & 0.763 & 0.760 & 0.754 & 242 \\
\hline
\end{tabular}

reported in Table 16 .

If we look into the decision metrics shown in Figure 23 for class "At Risk" (0), 82 out 86 observations are correctly predicted. Only 4 of the instances of this class are predicted to be at "Low Risk" (1). While 28 out of 77 instances of class "Low Risk" (1) is predicted as "At Risk" (0). For class "Healthy" (2), again 7 out of 79 samples are predicted to be as "Low Risk". Class "Healthy" (2) has high precision, while class "At Risk" has the highest recall, resulting in higher F1-score for classes "At Risk" and "Healthy", respectively. SVM has average $\mathrm{AUC}=0.87$, which means that there are $87 \%$ chances that it is able to distinguish between positive and negative classes. The AUC score per-class level, class "Healthy" and "At Risk" have the same score of 0.94 , which means that this model can identify the sample of these classes $94 \%$ of the time. For class "Low Risk", it is lower than 0.61.

CART Decision tree classifier achieves an accuracy and average F1-score of 0.983 as shown in the classification results in Table 17. Recall for class "At Risk" is 1, which means that this model can correctly identify all samples of this class. It is also shown in the confusion metrics for this model that 82 out of 82 samples are identified correctly. For class "Low Risk", 73 out of 77 are predicted positive. The precision for this class is 1 which means that all the 73 positive predicted are correctly identified. 

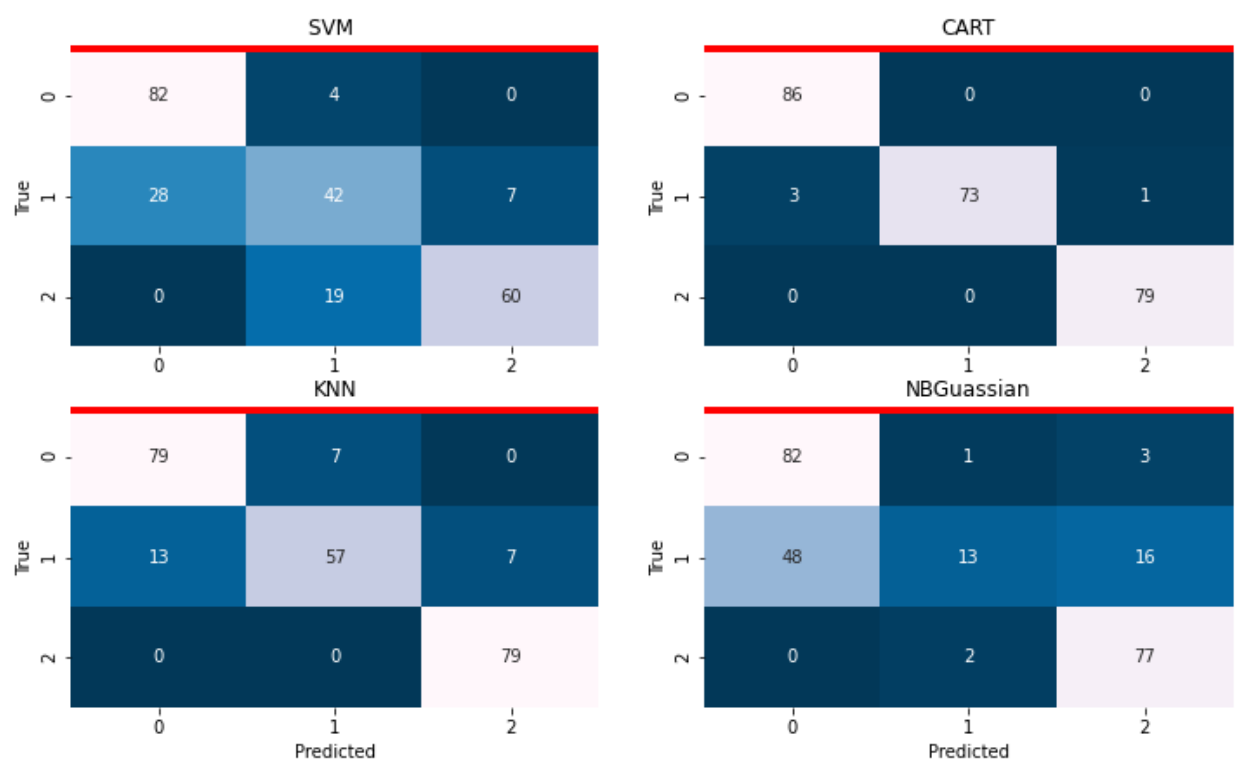

Figure 23: Decision metrics for classifiers.

For class "Healthy" again recall is 1, which means that this model can correctly identify all samples of this class. It is also validated by its confusion matrix which says 79 out 79 are predicted correctly. Overall, F1-score is the highest for class "Healthy". If we look into the average values, we observe no significant difference between the different metrics.

The ROC for CART is shown in Figure 24. The micro average AUC score is 0.98, which means that this model is $98 \%$ correct in the distinguishing between different classes. The AUC score for class "Healthy" and "At Risk" is 0.99, while it is lower for class "Low Risk" having value 0.95.

KNN: KNN achieves an accuracy and average weighted F1-score of 0.88. Recall for class "Healthy" is 1 , which means that this model can correctly identify all samples of this class. It is also shown in the confusion matrix in Figure 23 for this model that 79 out of 79 samples are identified correctly. This model have the same accuracy, weighted precision, weighted recall of $0.88, \mathrm{~F} 1$-score is slightly lower with value 0.885 . 

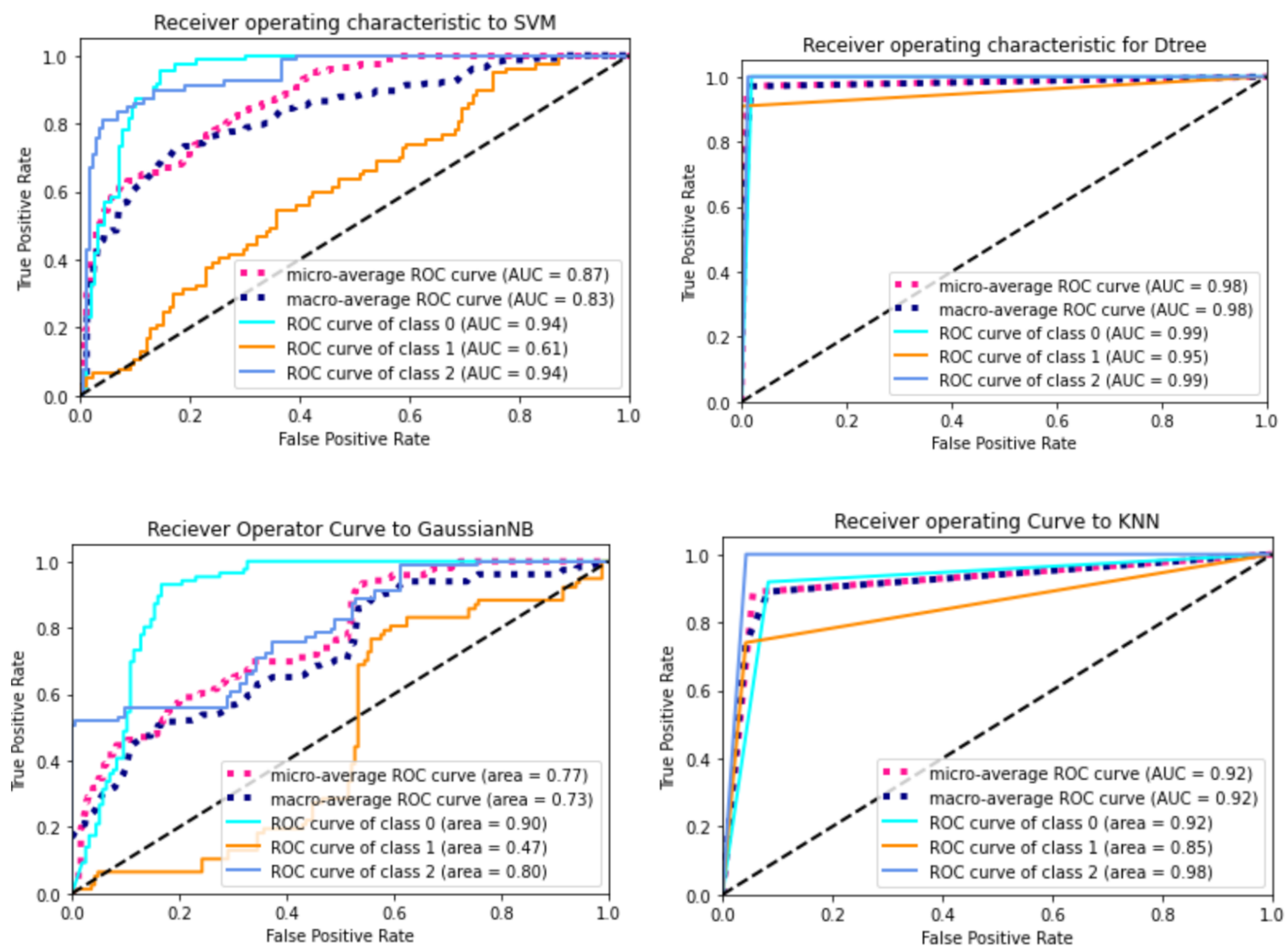

Figure 24: ROC curves.

Table 17: CART classification report.

\begin{tabular}{l|cccc}
\hline & Precision & Recall & F1-score & Support \\
\hline 0 & 0.966 & 1.000 & 0.983 & 86 \\
1 & 1.000 & 0.948 & 0.973 & 77 \\
2 & 0.988 & 1.000 & 0.994 & 79 \\
Accuracy & & & 0.983 & 242 \\
Macro average & 0.985 & 0.983 & 0.983 & 242 \\
Weighted average & 0.984 & 0.983 & 0.983 & 242 \\
\hline
\end{tabular}


Table 18: KNN classification results.

\begin{tabular}{l|cccc}
\hline & Precision & Recall & F1-score & Support \\
\hline 0 & 0.859 & 0.919 & 0.888 & 86 \\
1 & 0.891 & 0.740 & 0.809 & 77 \\
2 & 0.919 & 1.000 & 0.958 & 79 \\
Accuracy & & & 0.888 & 242 \\
Macro avg & 0.889 & 0.886 & 0.885 & 242 \\
Weighted avg & 0.888 & 0.888 & 0.885 & 242 \\
\hline
\end{tabular}

The micro-average AUC score for KNN is equal to 0.92. The AUC score for perclass is $0.92,0.85,0.98$ for class "At Risk", "Low Risk" and "Healthy", respectively.

Naive Bayes: Naive Bayes classifier has obtained an accuracy of 0.711 and weighted average F1-score 0.640. Recall is highest for class "Healthy" which is 0.975 , because it predicts 77 out of 79 samples correctly from this class. Recall for "Low Risk" for this model is 0.169 , which means that it did poorly to predict the instances of this class correctly. As shown in the confusion matrix in Figure 23, only 13 out of 77 are predicted correctly. While precision for this class is much higher than recall value, i.e., 0.182 . As shown in the confusion matrix, it predicted 13 out 16 values are predicted positive which are correct. This model achieves a higher precision, recall and F1-score for class "Healthy".

According to ROC of this model as shown in Figure 24, the AUC=047 less than 0.5 , which means that it ranks a random positive sample of this higher than a random negative sample less than $50 \%$ of the time. Which makes this classifier worse than a random guess classifier. The micro-average AUC score is 0.77 which is reasonable, but it is not doing good for class "Low Risk". 
Table 19: Naive Bayes classification results.

\begin{tabular}{l|cccc}
\hline & Precision & Recall & F1-score & Support \\
\hline 0 & 0.631 & 0.953 & 0.759 & 86 \\
1 & 0.812 & 0.169 & 0.280 & 77 \\
2 & 0.802 & 0.975 & 0.880 & 79 \\
Accuracy & & & 0.711 & 242 \\
Macro avg & 0.748 & 0.699 & 0.640 & 242 \\
Weighted avg & 0.745 & 0.711 & 0.646 & 242 \\
\hline
\end{tabular}

\section{Models' Explainability}

In this section, we present the results from calculating the permutation feature importance, discussed in Section 4.4.7, for all four classifiers.

Figure 25(a) shows the result for SVM classifier on the test set. As we can see that permuting "age_in_weeks" effected the AUC score the most, followed by "complexity". The minimal loss resulted by permuting "num_commits" and "token_count". For decision trees, as shown in Figure 25(b), "age_in_weeks" remains the most important feature, but "watchers", "forks", "complexity", and "total_issues" have nearly similar importance. Moreover, "nloc", "lines_removed", and "method_count" incurred 0 loss after permutation, so the model has totally ignored these features while predicting the health of a project. While in KNN, as shown in Figure 25(c), "age_in_weeks" remains the most important feature, followed by "nloc"; this result is unlike the 0 importance of "nloc" in the decision tree model. KNN uses all the features in its prediction model as none of the features have 0 importance, with "watchers" and "forks" being the least important. Similarly, in Naive Bayes 25(d) "age_in_weeks" remains the most important feature, which demonstrates a decrease of 0.1 in AUC score, followed by "total_issues", while after permuting "number_commits" the AUC score remains unchanged. 


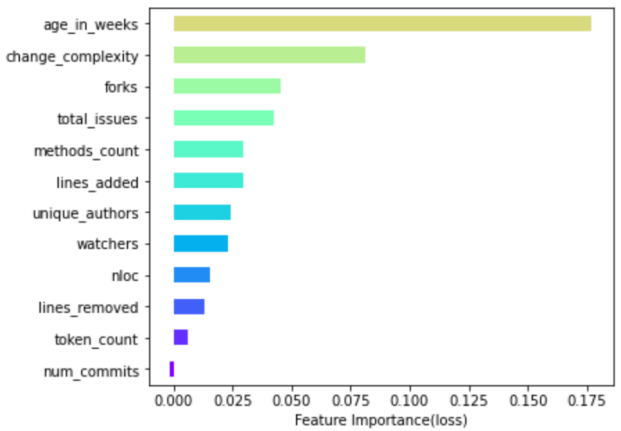

(a) SVM

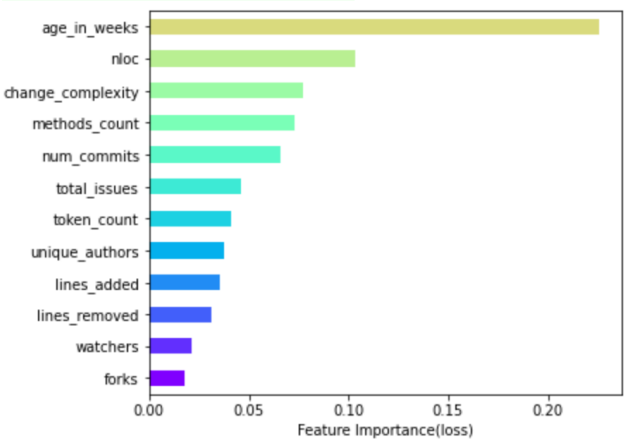

(c) KNN

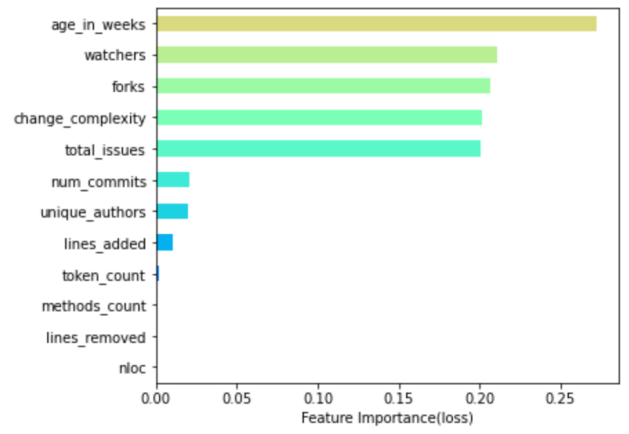

(b) Decision tree

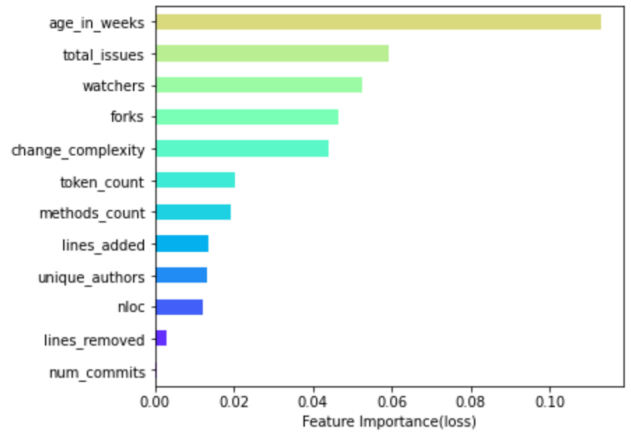

(d) Naive Bayes

Figure 25: Feature permutation with increase in loss. 


$\begin{aligned} \text { Weight } & \text { Feature } \\ 0.1771 \pm 0.0290 & \text { age_in_weeks } \\ 0.0812 \pm 0.0172 & \text { change_complexity } \\ 0.0454 \pm 0.0128 & \text { forks } \\ 0.0426 \pm 0.0273 & \text { total_issues } \\ 0.0298 \pm 0.0097 & \text { methods_count } \\ 0.0297 \pm 0.0169 & \text { lines_added } \\ 0.0245 \pm 0.0250 & \text { unique_authors } \\ 0.0230 \pm 0.0227 & \text { watchers } \\ 0.0153 \pm 0.0075 & \text { nloc } \\ 0.0133 \pm 0.0058 & \text { lines_removed } \\ 0.0059 \pm 0.0089 & \text { token_count } \\ -0.0015 \pm 0.0161 & \text { num_commits }\end{aligned}$

(a) SVM

$\begin{aligned} \text { Weight } & \text { Feature } \\ 0.2255 \pm 0.0354 & \text { age_in_weeks } \\ 0.1035 \pm 0.0319 & \text { nloc } \\ 0.0769 \pm 0.0234 & \text { change_complexity } \\ 0.0729 \pm 0.0294 & \text { methods_count } \\ 0.0661 \pm 0.0240 & \text { num_commits } \\ 0.0461 \pm 0.0201 & \text { total_issues } \\ 0.0410 \pm 0.0211 & \text { token_count } \\ 0.0374 \pm 0.0230 & \text { unique_authors } \\ 0.0356 \pm 0.0229 & \text { lines_added } \\ 0.0310 \pm 0.0232 & \text { lines_removed } \\ 0.0211 \pm 0.0133 & \text { watchers } \\ 0.0176 \pm 0.0128 & \text { forks }\end{aligned}$

(c) $\mathrm{KNN}$

$\begin{aligned} \text { Weight } & \text { Feature } \\ 0.2721 \pm 0.0397 & \text { age_in_weeks } \\ 0.2112 \pm 0.0341 & \text { watchers } \\ 0.2070 \pm 0.0371 & \text { forks } \\ 0.2017 \pm 0.0335 & \text { change_complexity } \\ 0.2003 \pm 0.0362 & \text { total_issues } \\ 0.0204 \pm 0.0124 & \text { num_commits } \\ 0.0200 \pm 0.0116 & \text { unique_authors } \\ 0.0108 \pm 0.0096 & \text { lines_added } \\ 0.0017 \pm 0.0042 & \text { token_count } \\ 0 \pm 0.0000 & \text { methods_count } \\ 0 \pm 0.0000 & \text { lines_removed } \\ 0 \pm 0.0000 & \text { nloc }\end{aligned}$

(b) Decision tree

$\begin{aligned} \text { Weight } & \text { Feature } \\ 0.1129 \pm 0.0353 & \text { age_in_weeks } \\ 0.0590 \pm 0.0381 & \text { total_issues } \\ 0.0523 \pm 0.0320 & \text { watchers } \\ 0.0466 \pm 0.0319 & \text { forks } \\ 0.0441 \pm 0.0176 & \text { change_complexity } \\ 0.0201 \pm 0.0136 & \text { token_count } \\ 0.0193 \pm 0.0136 & \text { methods_count } \\ 0.0137 \pm 0.0140 & \text { lines_added } \\ 0.0130 \pm 0.0267 & \text { unique_authors } \\ 0.0120 \pm 0.0136 & \text { nloc } \\ 0.0031 \pm 0.0104 & \text { lines_removed } \\ 0.0004 \pm 0.0087 & \text { num_commits }\end{aligned}$

(d) Naive Bayes

Figure 26: Weights after feature permutation. 
In Figure 26, the first number in each row shows the reduction in the model's performance, in this case AUC score, by the reshuffling of that feature. The second number is a measure of the randomness of the performance reduction for different reshuffles of the feature column. It measures how performance varied from onereshuffling to the next.

Overall, "age_in_weeks" is the most important feature across all the four classifiers, but the rest of the features vary for each model. Moreover, the maximum decrease in the performance of the estimators is equal to 0.2 , which is for KNN and decision tree. The difference between the incurred loss of the first five features in the decision tree is minimal, meaning that the model uses these features equally to predict the health of a project. "num_commits" is the least important feature for two classifiers, Naive Bayes and SVM, followed by the features that are correlated with "num_commits" such as "lines_removed", "nloc" and "token_count".

Answer to RQ3: According to our machine learning classification results, the decision tree classifier demonstrates better performance when predicting the health of the Bitcoin ecosystem followed by the KNN classifier. We achieved the accuracy of 0.98 and 0.88 for the decision tree and KNN classifier, respectively. Age of the project, i.e., "age_in_weeks", is found to be the most important feature across all the four classifiers. 


\section{Chapter 6}

\section{Discussion}

In this chapter, we discuss the main findings of our study (Section 6.1), implications of our work for the researchers in the field of mining GitHub data (Section 6.2), Bitcoin community (Section 6.3), and researchers working in the field of software ecosystems' health (Section 6.4). Section 6.5 addresses the threats to validity of the study.

\subsection{Main Findings}

The Bitcoin ecosystem in GitHub is limited compared to the "big picture" of the Bitcoin ecosystem. For example, there are over million web and mobile wallet apps available, yet only 51 wallet projects are actively developed and maintained in GitHub. The same observation also applies to the other eight categories of the ecosystem. Moreover, the documentation category has only emerged in GitHub in 2014, meaning that developers have started to work and share documentation related projects. Similarly, the data analysis category has emerged in 2016. As the field of data analytics has matured, the developer community has showed interest in analyzing Bitcoin data and predictive modeling.

The results from our manual categorization and clustering are quite different. While we considered seven most important variables during clustering such as the 
method count, code complexity, age, number of issues, number of commits, nloc, forks and number of contributors, in our manual categorization we grouped the projects based on their description and README files. Besides, in terms of health, the majority of projects are falling into "Low Risk" class. We can say that not all of the projects are performing well in all the defined health metrics. Moreover, over the years the ecosystem has changed significantly. For example, between 2010 and 2013, there were just five categories such as node, wallet, mining, development and services which are essential software to the Bitcoin function. After 2014, each year a new category has emerged in the ecosystem.

\subsection{Implications for the Bitcoin Community in GitHub}

The Bitcoin development community is the first audience to this research work. We have a number of recommendation to offer for the Bitcoin developers.

First, we noticed that the number of contributors seem to be decreasing each year. According to Wahyudin et al. [168], in a healthy and sustainable software ecosystem developers contribute to different projects, but problems arise when they leave the ecosystem. To increase sustainability of the ecosystem, a number of actions can be applied to increase the retention rates of the developers in the ecosystem. Researchers in the field of software development have suggested different ways to increase retention rates. A study by Constantinou et al. [169] reveals that developers retention can be improved when contributors remain active and have strong contribution intensity, regardless of whether they are core or peripheral developers. Similarly, Lin et al. [170 find through survival analysis that developers who join a project earlier have higher chances to stay longer, thus they suggest that encouraging newcomers may effect 
their surviablity in the project. Moreover, they suggest that balancing collaboration and individualism may help increase retention rate. The developers who code may be assigned some documentation tasks, while developers in charge of documentation should not only take on documentation tasks, but also some coding tasks to increase their chances of staying on the project. Furthermore, Midha et al. [171] find that high modularity increases the retention rate, while high complexity lowers the retention rate in open-source projects. Their results indicate that both modularity and complexity have high influence on developer retention rates.

The number of contributors depends on the number open source projects, or generally openness of the software in the ecosystem. A potential solution to attract more developers, the community should make their projects open source. As openness is directly correlated with software reliability, a more transparent flow of information can foster larger number of developers in the ecosystem [172].

Second, we found only six projects that are related to Bitcoin documentation. To improve code quality, as well as better developer understanding of community's goals, more efforts should be dedicated to writing various kinds of documentation related to code (e.g., code review, code standards), community (existing policies, code of conduct, policies, vision, etc) and more.

Third, only $12 \%$ of the developers are contributing to more than one category. The Bitcoin ecosystem should facilitate better knowledge transfer across different categories to be able to maintain a sustainable software ecosystem. Perhaps having certain initiatives in place, such as a dedicated group of users who can oversee the overall health and growth of the ecosystem and implement certain policies and practices for more sustainable future of the Bitcoin ecosystem within GitHub. 


\subsection{Implications for the Bitcoin Community}

Here, we provide a few potentially useful recommendations to the Bitcoin community in general. The Bitcoin community is very connected and regulates the Bitcoin cryptocurrency without the involvement of the government or any third party. Different stakeholders communicate through online forums and social media such as Facebook and Twitter. In this study, we found that there is nearly a lack of collaboration between developers within the GitHub on the projects that comprise the ecosystem. Most of the developers in the ecosystem contribute to a single project within one category. There is very little knowledge transfer across different categories. For example, wallet developers may contribute to other categories such as development, payment and perhaps services, while contributors in the development category can participate in more specialized projects such as wallets. Knowledge transfer and expertise development are critical aspects of the sustainable communities.

Furthermore, investors should consider the overall health of the Bitcoin OSSE before making any investment decisions. This include both technical and social aspects of the OSSE. For example, they should investigate who the core developers are and how the cryptocurrency would work if the key developers suddenly leave the project. QuadrigaCX $\sqrt{173}$ is an example of such a case; investors have lost millions of dollars when the QuadrigaCX co-founder and key developer Gerald Cotten has suddenly disappeared. In the case of Bitcoin, the health of the main Bitcoin repository on GitHub should be carefully considered prior to investing. Good health indicators of the ecosystem become more critical for smaller cryptocurrencies as the risk of investment is higher. 


\subsection{Implications for Research Community}

There are no standard and well defined health metrics used by the researchers to measure the health of a software ecosystem. Most of the researchers refer to social metrics $27,78,85,86]$. We can be enrich these metrics by considering source code quality metrics. In this study, we showed that code quality metrics such as CC can be useful in measuring and predicting the health of an ecosystem. We believe that code quality metrics together with social metrics can be a powerful tool in evaluating the health of a software project or an ecosystem.

We have defined the Bitcoin ecosystem in GitHub using our knowledge of a more general definition of the Bitcoin ecosystem. Many researchers have used other common ways such as package dependency and project relevance $[7,25,25,26,54,55$. However, for ecosystems that may include projects with no known package dependency or relevance such as in the example of the Bitcoin ecosystem, what approach should be followed for identifying such ecosystems and their boundaries. How one can define an OSSE around a potential "community" that lacks any information, documentation, code dependency, etc.? We believe that research community should continue their efforts in establishing and developing techniques for identifying software ecosystems, assessing and evaluating their health. There is no "one fits all" definition of an ecosystem, and future studies of various ecosystems in GitHub can offer better insights into a diversity of the ecosystems out there.

\subsection{Threats to Validity}

Our study is subject to several threats to validity. One potential threat can be the quality of the GHTorrent dataset. We used Big Query to create a list of Bitcoin projects, and filtered the list based on the conditions discussed in Section 4.1. During 
the manual classification, we noticed that there are some projects in the GitHub that GHTorrent have missed to collect in its SQL database. Moreover, while cloning and extracting the source code features, we observed a difference between the number of commits we aggregated form GHTorrent and the number we extracted by cloning repositories. We have mitigated the GHTorrent quality by collecting our metrics querying "live" GitHub data.

Along with threats to the correctness of the dataset, there are many other threats to the to the quality of study design and rigorousness of its execution. First, the heuristic based approach we adopted to classify GitHub projects in different classes of health can be another potential threat to the validity.

Second, we only have data for four months of the year 2018. In the trends analysis this data is projected for 12 months.

Human bias and error during manual categorization for RQ1, can also be a potential threat. To mitigate this bias, two human subjects were involved in classifying projects on GitHub into nine categories.

Fourth, our categorization of the Bitcoin projects is dated to 01-04-2018. As time passes by, we believe more and more projects would emerge in GitHub organized around various and potentially new categories within the ecosystem.

Another treat is related to the choice of classification models. We have not explored all possible classifiers but rather focused on the most popular ones such as KNN, Naive Bayes, decision trees, etc. 


\section{Chapter 7}

\section{Conclusions}

We conclude our work with a summary of our contributions in Section 7.1 and define some future research directions in Section 7.2,

\subsection{Summary of Contributions}

In this thesis, we have studied the Bitcoin software ecosystem in GitHub and explored its health. This work makes seven contributions to the research communities in different fields. First, we define the Bitcoin software ecosystem in GitHub. To better understand the ecosystem, we categorized the projects related to the Bitcoin cryptocurrency into different applications of Bitcoin. We applied both manual and data-driven classification approaches. The nine categories from manual classification appear to represent the Bitcoin ecosystem in GitHub compared to its more general and known definition. Second, we have collected the source code metrics by cloning each project in the dataset and extracting the aggregated values. Third, we have defined the health metrics by leveraging both the social and technical features of a project. Fourth, we classified the projects in the dataset into three classes of health such as "Healthy", "Low Risk", and "At Risk". Fifth, we analyzed the trends in the health of the ecosystem as a whole. We performed the analysis over the span of 
eight years and across different categories. As our sixth contribution, we used the health labels to build four classification models to predict the health of a project. Moreover, we compared the performance of the different classifiers to determine the best classifier for our data and problem. We found that decision trees outperform other classifiers such as KNN, SVM and Naive Bayes.

Finally, we discussed implications to three different groups of audiences, who can benefit from this line of research: Bitcoin developers in GitHub, researchers in the field of cryptocurrency, and the software ecosystem community.

\subsection{Future Work}

There are a number of research opportunities that can be built on top of this work. First, we can analyze the discussion around the evaluation of pull requests to understand the reasons for the loss of contributors in the ecosystem. How often pull requests are accepted by contributors? Who is in charge of making these decisions? Does this decision making affect contributor loss and retention rates?

Second, it would be critical to conduct qualitative studies (surveys, interviews) with Bitcoin developers to better understand how and why they decide to contribute or not to the projects hosted in GitHub.

Another follow-up could be to combine data from a social media platform such as Twitter to analyze whether online discussions affect the health of the ecosystem, in particular, the loss of contributors.

In our work we have only studied Bitcoin related projects that are hosted in GitHub. It would be interesting to expand the search for the Bitcoin projects that are hosted and shared elsewhere (e.g., mobile wallet apps). Including projects nonGitHub to the analysis can result in the expansion of the Bitcoin ecosystem and bring more insights into how developers collaborate on the Bitcoin development via several 
different platforms.

Another potential line of research may study the effects of financial markets and social media platforms and trends on the ecosystem's health. There has been an extensive research on how social media may influence the price of the Bitcoin cryptocurrency [174,175], or the stock prices in general [176, 177], as well as numerous attempts in predicting the price of the cryptocurrency. For example, Mai et al. [174 studied how social media impacts Bitcoin value. They found that overall social media sentiment is a crucial element in valuation of Bitcoin, but all the social media platforms do not have the same impact. Similarly, Xie et al. [175] studied the relationship between Bitcoin price movement and social media discussions. They compared the predictive power of the discussions across different channels. Balfagih et al. [178 conducted a study on Bitcoin Twitter feeds in order to predict it price. 


\section{List of References}

[1] S. Jansen, "Measuring the health of open source software ecosystems: Beyond the scope of project health," Information and Software Technology, vol. 56, no. 11, pp. 1508-1519, 2014.

[2] D. Williams, Cryptocurrency Compendium: A Reference for Digital Currencies. Lulu.com, 2017.

[3] J. Carrick, "Bitcoin as a complement to emerging market currencies," Emerging Markets Finance and Trade, vol. 52, no. 10, pp. 2321-2334, 2016.

[4] S. Dallyn, "Cryptocurrencies as market singularities: the strange case of Bitcoin," Journal of Cultural Economy, vol. 10, no. 5, pp. 462-473, 2017.

[5] D. G. Messerschmitt and C. Szyperski, "Software ecosystems: understanding an indispensable technology and industry. 2003," 2003.

[6] K. Manikas and K. M. Hansen, "Software ecosystems-A systematic literature review," Journal of Systems and Software, vol. 86, no. 5, pp. 1294-1306, 2013.

[7] S. Jansen, A. Finkelstein, and S. Brinkkemper, "A sense of community: A research agenda for software ecosystems," in 2009 31st International Conference on Software Engineering-Companion Volume, pp. 187-190, IEEE, 2009.

[8] "Eli5-scikit-learn." https://cointelegraph.com/news/ understanding-the-bitcoin-ecosystem. Accessed: 2019-09-30.

[9] G. Hileman and M. Rauchs, "Global cryptocurrency benchmarking study," Cambridge Centre for Alternative Finance, vol. 33, 2017.

[10] S. Nakamoto et al., "Bitcoin: A peer-to-peer electronic cash system," 2008.

[11] "Coin Market Cap." https://coinmarketcap.com. Accessed: 2010-09-30. 
[12] "99Bitcoin." https://99bitcoins.com/bitcoin/who-accepts. Accessed: 2010-09-30.

[13] "Microsoft." https://www.microsoft.com/en-ca/. Accessed: 2010-12-30.

[14] "Amazon." https://www.amazon.ca/. Accessed: 2010-12-30.

[15] "Amazon." https://purse.io/shop. Accessed: 2010-12-30.

[16] "Wikipedia." https://en.wikipedia.org/wiki/Main_Page. Accessed: 2010$12-30$.

[17] "Expedia." https://www.expedia.ca/. Accessed: 2010-12-30.

[18] "Overstock." https://www.overstock.com/. Accessed: 2010-12-30.

[19] "BitPay." https://bitpay.com/. Accessed: 2010-12-30.

[20] "Coinbase." https://www. coinbase.com/. Accessed: 2019-12-30.

[21] T. Mens, M. Claes, P. Grosjean, and A. Serebrenik, "Studying evolving software ecosystems based on ecological models," in Evolving Software Systems, pp. 297326, Springer, 2014.

[22] M. A. Cusumano, "The Bitcoin Ecosystem," Communications of the ACM, vol. 57, no. 10, pp. 22-24, 2014.

[23] I. Van Den Berk, S. Jansen, and L. Luinenburg, "Software ecosystems: a software ecosystem strategy assessment model," in Proceedings of the Fourth European Conference on Software Architecture: Companion Volume, pp. 127-134, 2010.

[24] A. Gawer, The Rise of the Platform Enterprise: A Global Survey. Center for Global Enterprise, 2016.

[25] M. Lungu, "Towards reverse engineering software ecosystems," in 2008 IEEE International Conference on Software Maintenance, pp. 428-431, IEEE, 2008.

[26] V. Cosentino, J. L. C. Izquierdo, and J. Cabot, "A systematic mapping study of software development with GitHub," IEEE Access, vol. 5, pp. 7173-7192, 2017.

[27] T. Mens, B. Adams, and J. Marsan, "Towards an interdisciplinary, socio-technical analysis of software ecosystem health," arXiv preprint arXiv:1711.04532, 2017. 
[28] "SlashDot."

https://slashdot.org/story/15/10/05/2031247/ linux-kernel-dev-sarah-sharp-quits-citing-brutal-communications-style, Accessed: 2015-01-12.

[29] "The npm Blog." https://blog.npmjs.org/post/141577284765/ kik-left-pad-and-npm. Accessed: 2016-03-12.

[30] J. Marsan, M. Templier, P. Marois, B. Adams, K. Carillo, and G. L. Mopenza, "Toward Solving Social and Technical Problems in Open Source Software Ecosystems: Using Cause-and-Effect Analysis to Disentangle the Causes of Complex Problems," IEEE Software, vol. 36, pp. 34-41, Jan 2019.

[31] M. A. Cusumano, "The bitcoin ecosystem," Communications of the ACM, vol. 57, no. 10, pp. 22-24, 2014.

[32] "Spark Capital." https://thegongshow.tumblr.com/post/64777314119/ bitcoin-market-map-spark-capital-was-interested/. Accessed: 2014-0312 .

[33] "Bitcoin Core Wallet." https://bitcoin.org/en/wallets/desktop/ windows/bitcoincore/. Accessed: 2019-09-30.

[34] "Samourai Bitcoin Wallet." https://samouraiwallet.com/, Accessed: 201909-30.

[35] "Armory Bitcoin Wallet." https://www.bitcoinarmory.com/. Accessed: 2019-09-30.

[36] "GreenAddress Bitcoin Wallet." https://greenaddress.it/en/. Accessed: 2019-09-30.

[37] "Ripple." https://ripple.com/. Accessed: 2014-03-12.

[38] "Bit Instant." https://bitinstant.io/. Accessed: 2019-03-12.

[39] "BIPS." https://en.bitcoin.it/wiki/BIPS. Accessed: 2019-03-12.

[40] "Local Bitcoins." https://localbitcoins.com/. Accessed: 2019-03-12.

[41] "BitStamp." https://www.bitstamp.net/. Accessed: 2019-03-12.

[42] "bitbond." https://www.bitbond.com. Accessed: 2020-01-30.

[43] "Bit-Lender." https://bit-lender.com/. Accessed: 2020-01-30. 
[44] "BUTTERFLY LABS." https://www.butterfly-labs.com/. Accessed: 2020-01-30.

[45] "Lamassu." https://lamassu.is/. Accessed: 2020-01-30.

[46] "Robocoin." https://coinatmradar.com/manufacturer/4/ robocoin-kiosk-bitcoin-atm-producer/. Accessed: 2020-01-30.

[47] "Hash Flair." http://hashflare.io/. Accessed: 2020-01-30.

[48] "SATOSHIDICE DAPP." https://satoshidice.com/. Accessed: 2020-01-30.

[49] "COINSTAR." https://www.coinstar.com/howcoinstarworks. Accessed: 2020-01-30.

[50] "BITCOINGET." https://www.bitcoinget.com/. Accessed: 2020-01-30.

[51] "BITPAY." https://bitpay.com/card/. Accessed: 2020-01-30.

[52] K. Manikas, "Revisiting software ecosystems research: A longitudinal literature study," Journal of Systems and Software, vol. 117, pp. 84-103, 2016.

[53] J. Bosch and P. Bosch-Sijtsema, "From integration to composition: On the impact of software product lines, global development and ecosystems," Journal of Systems and Software, vol. 83, no. 1, pp. 67-76, 2010.

[54] S. Jansen, S. Brinkkemper, and A. Finkelstein, "Business Network Management as a Survival Strategy: A Tale of Two Software Ecosystems," Iwseco@ Icsr, vol. 2009, 2009.

[55] J. Bosch, "From software product lines to software ecosystems," in $S P L C$, vol. 9, pp. 111-119, 2009.

[56] M. Lungu, R. Robbes, and M. Lanza, "Recovering inter-project dependencies in software ecosystems," in Proceedings of the IEEE/ACM international conference on Automated software engineering, pp. 309-312, ACM, 2010.

[57] A. Serebrenik and T. Mens, "Challenges in software ecosystems research," in Proceedings of the 2015 European Conference on Software Architecture Workshops, pp. 1-6, 2015.

[58] O. Franco-Bedoya, D. Ameller, D. Costal, and X. Franch, "Open source software ecosystems: A Systematic mapping," Information and Software Technology, vol. 91, pp. 160-185, 2017. 
[59] J. Knodel and K. Manikas, "Towards a typification of software ecosystems," in International Conference of Software Business, pp. 60-65, Springer, 2015.

[60] S. Hyrynsalmi, J. Ruohonen, and M. Seppänen, "Healthy until otherwise proven: some proposals for renewing research of software ecosystem health," in 2018 IEEE/ACM 1st International Workshop on Software Health (SoHeal), pp. 18-24, IEEE, 2018.

[61] F. Perez, B. E. Granger, and J. D. Hunter, "Python: an ecosystem for scientific computing," Computing in Science \& Engineering, vol. 13, no. 2, pp. 13-21, 2010.

[62] W. Ma, L. Chen, Y. Zhou, and B. Xu, "What Are the Dominant Projects in the GitHub Python Ecosystem?," in 2016 Third International Conference on Trustworthy Systems and their Applications (TSA), pp. 87-95, IEEE, 2016.

[63] M. Claes, T. Mens, and P. Grosjean, "On the maintainability of CRAN packages," in 2014 Software Evolution Week-IEEE Conference on Software Maintenance, Reengineering, and Reverse Engineering (CSMR-WCRE), pp. 308-312, IEEE, 2014.

[64] D. M. German, B. Adams, and A. E. Hassan, "The evolution of the R software ecosystem," in 2013 17th European Conference on Software Maintenance and Reengineering, pp. 243-252, IEEE, 2013.

[65] B. Vasilescu, A. Serebrenik, P. Devanbu, and V. Filkov, "How social Q\&A sites are changing knowledge sharing in open source software communities," in Proceedings of the 17th ACM conference on Computer supported cooperative work 6 social computing, pp. 342-354, ACM, 2014.

[66] R. Robbes, M. Lungu, and D. Röthlisberger, "How do developers react to api deprecation?: the case of a smalltalk ecosystem," in Proceedings of the ACM SIGSOFT 20th International Symposium on the Foundations of Software Engineering, p. 56, ACM, 2012.

[67] J. Businge, A. Serebrenik, and M. van den Brand, "Survival of Eclipse thirdparty plug-ins," in 2012 28th IEEE International Conference on Software Maintenance (ICSM), pp. 368-377, IEEE, 2012.

[68] J. Businge, A. Serebrenik, and M. van den Brand, "Analyzing the Eclipse API usage: Putting the developer in the loop," in 2013 17th European Conference on Software Maintenance and Reengineering, pp. 37-46, IEEE, 2013. 
[69] J. C. Dueñas, F. Cuadrado, M. Santillán, J. L. Ruiz, et al., "Apache and Eclipse: Comparing open source project incubators," IEEE software, vol. 24, no. 6, pp. 90-98, 2007.

[70] T. Mens, J. Fernández-Ramil, and S. Degrandsart, "The evolution of Eclipse," in 2008 IEEE International Conference on Software Maintenance, pp. 386-395, IEEE, 2008.

[71] M. Harman, Y. Jia, and Y. Zhang, "App store mining and analysis: MSR for app stores," in Proceedings of the 9th IEEE Working Conference on Mining Software Repositories, pp. 108-111, IEEE Press, 2012.

[72] S. Hyrynsalmi, T. Mäkilä, A. Järvi, A. Suominen, M. Seppänen, and T. Knuutila, "App store, marketplace, play! an analysis of multi-homing in mobile software ecosystems," Jansen, Slinger, pp. 59-72, 2012.

[73] N. Bettenburg, A. E. Hassan, B. Adams, and D. M. German, "Management of community contributions," Empirical Software Engineering, vol. 20, no. 1, pp. 252-289, 2015.

[74] E. Constantinou, A. Decan, and T. Mens, "Breaking the borders: an investigation of cross-ecosystem software packages," arXiv preprint arXiv:1812.04868, 2018.

[75] J. Teixeira and S. Hyrynsalmi, "How Do Software Ecosystems Co-Evolve?," in International Conference of Software Business, pp. 115-130, Springer, 2017.

[76] S. Abramova and R. Böhme, "Perceived benefit and risk as multidimensional determinants of bitcoin use: a quantitative exploratory study," 2016.

[77] D. Y. Huang, H. Dharmdasani, S. Meiklejohn, V. Dave, C. Grier, D. McCoy, S. Savage, N. Weaver, A. C. Snoeren, and K. Levchenko, "Botcoin: Monetizing Stolen Cycles.," in NDSS, Citeseer, 2014.

[78] T. Mens, M. Claes, and P. Grosjean, "ECOS: Ecological studies of open source software ecosystems," in 2014 Software Evolution Week-IEEE Conference on Software Maintenance, Reengineering, and Reverse Engineering (CSMR-WCRE), pp. 403-406, IEEE, 2014.

[79] B. Vasilescu, A. Serebrenik, M. Goeminne, and T. Mens, "On the variation and specialisation of workload - a case study of the Gnome ecosystem community," Empirical Software Engineering, vol. 19, no. 4, pp. 955-1008, 2014. 
[80] A. Decan, T. Mens, M. Claes, and P. Grosjean, "When GitHub meets CRAN: An analysis of inter-repository package dependency problems," in 2016 IEEE 23rd International Conference on Software Analysis, Evolution, and Reengineering (SANER), vol. 1, pp. 493-504, IEEE, 2016.

[81] A. Decan, T. Mens, and M. Claes, "An empirical comparison of dependency issues in OSS packaging ecosystems," in 2017 IEEE 24th International Conference on Software Analysis, Evolution and Reengineering (SANER), pp. 2-12, IEEE, 2017.

[82] E. Constantinou and T. Mens, "Socio-Technical Evolution of the Ruby Ecosystem in GitHub," 022017.

[83] E. Constantinou and T. Mens, "Social and technical evolution of software ecosystems: A case study of Rails," in Proccedings of the 10th European Conference on Software Architecture Workshops, pp. 1-4, 2016.

[84] M. Claes, T. Mens, R. Di Cosmo, and J. Vouillon, "A historical analysis of debian package incompatibilities," in 2015 IEEE/ACM 12th Working Conference on Mining Software Repositories, pp. 212-223, IEEE, 2015.

[85] T. Mens, "An ecosystemic and socio-technical view on software maintenance and evolution," in 2016 IEEE International Conference on Software Maintenance and Evolution (ICSME), pp. 1-8, IEEE, 2016.

[86] K. Carillo, J. Marsan, and B. Negoita, "Exploring the biosphere-Towards a conceptualization of peer production communities as living organisms," in Proc. AIS SIGOPEN Developmental Workshop for Research on Open Phenomena, pp. 1-12, 2017.

[87] J. Dijkers, R. Sincic, N. Wasankhasit, and S. Jansen, "Exploring the effect of software ecosystem health on the financial performance of the open source companies," in 2018 IEEE/ACM 1st International Workshop on Software Health (SoHeal), pp. 48-55, IEEE, 2018.

[88] R. J. Wieringa, Design science methodology for information systems and software engineering. Springer, 2014.

[89] G. Gousios, "The GHTorrent dataset and tool suite," in Proceedings of the 10th Working Conference on Mining Software Repositories, MSR '13, (Piscataway, NJ, USA), pp. 233-236, IEEE Press, 2013. 
[90] D. Alami, M. Rodríguez, and S. Jansen, "Relating health to platform success: exploring three e-commerce ecosystems," in Proceedings of the 2015 European Conference on Software Architecture Workshops, pp. 1-6, 2015.

[91] L. Soussi, Z. Spijkerman, and S. Jansen, "A case study of the health of an augmented reality software ecosystem: Vuforia," in International Conference of Software Business, pp. 145-152, Springer, 2016.

[92] J. Axelsson and M. Skoglund, "Quality assurance in software ecosystems: A systematic literature mapping and research agenda," Journal of Systems and Software, vol. 114, pp. 69-81, 2016.

[93] S. Hyrynsalmi, M. Seppänen, T. Nokkala, A. Suominen, and A. Järvi, "Wealthy, Healthy and/or Happy - What does 'ecosystem health'stand for?," in International Conference of Software Business, pp. 272-287, Springer, 2015.

[94] K. Manikas and K. M. Hansen, "Reviewing the health of software ecosystems-a conceptual framework proposal," in Proceedings of the 5th international workshop on software ecosystems (IWSECO), pp. 33-44, Citeseer, 2013.

[95] S. da Silva Amorim, F. S. S. Neto, J. D. McGregor, E. S. de Almeida, and C. von Flach G. Chavez, "How has the health of software ecosystems been evaluated? A systematic review," in Proceedings of the 31st Brazilian symposium on software engineering, pp. 14-23, 2017.

[96] D. G. Baur, K. Hong, and A. D. Lee, "Bitcoin: Medium of exchange or speculative assets?," Journal of International Financial Markets, Institutions and Money, vol. 54, pp. 177-189, 2018.

[97] Z. Li, Q. Lu, S. Chen, Y. Liu, and X. Xu, "A Landscape of Cryptocurrencies," in 2019 IEEE International Conference on Blockchain and Cryptocurrency $(I C B C)$, pp. 165-166, IEEE, 2019.

[98] J. Abraham, D. Higdon, J. Nelson, and J. Ibarra, "Cryptocurrency price prediction using tweet volumes and sentiment analysis," SMU Data Science Review, vol. 1, no. 3, p. 1, 2018.

[99] A. Mittal, V. Dhiman, A. Singh, and C. Prakash, "Short-Term Bitcoin Price Fluctuation Prediction Using Social Media and Web Search Data," in 2019 Twelfth International Conference on Contemporary Computing (IC3), pp. 1-6, IEEE, 2019. 
[100] A. Burnie and E. Yilmaz, "An Analysis of the Change in Discussions on Social Media with Bitcoin Price," in Proceedings of the 42nd International ACM SIGIR Conference on Research and Development in Information Retrieval, pp. 889-892, 2019.

[101] M. Balcilar, E. Bouri, R. Gupta, and D. Roubaud, "Can volume predict Bitcoin returns and volatility? A quantiles-based approach," Economic Modelling, vol. 64 , pp. $74-81,2017$.

[102] E. Kalliamvakou, D. Damian, K. Blincoe, L. Singer, and D. M. German, "Open source-style collaborative development practices in commercial projects using GitHub," in 2015 IEEE/ACM 37th IEEE International Conference on Software Engineering, vol. 1, pp. 574-585, IEEE, 2015.

[103] G. Gousios and D. Spinellis, "GHTorrent: GitHub's data from a firehose," in 2012 9th IEEE Working Conference on Mining Software Repositories (MSR), pp. 12-21, IEEE, 2012.

[104] J. E. Montandon, L. L. Silva, and M. T. Valente, "Identifying experts in software libraries and frameworks among GitHub users," in 2019 IEEE/ACM 16th International Conference on Mining Software Repositories (MSR), pp. 276-287, IEEE, 2019.

[105] J. Tsay, L. Dabbish, and J. Herbsleb, "Let's talk about it: evaluating contributions through discussion in GitHub," in Proceedings of the 22nd ACM SIGSOFT international symposium on foundations of software engineering, pp. 144-154, 2014 .

[106] S. S. Manes and O. Baysal, "How often and what StackOverflow posts do developers reference in their GitHub projects?," in 2019 IEEE/ACM 16th International Conference on Mining Software Repositories (MSR), pp. 235-239, IEEE, 2019.

[107] C. Treude and M. Wagner, "Predicting good configurations for GitHub and stack overflow topic models," in 2019 IEEE/ACM 16th International Conference on Mining Software Repositories (MSR), pp. 84-95, IEEE, 2019.

[108] A. Trockman, R. van Tonder, and B. Vasilescu, "Striking gold in software repositories? an econometric study of cryptocurrencies on github," in 2019 IEEE/ACM 16th International Conference on Mining Software Repositories (MSR), pp. 181-185, IEEE, 2019. 
[109] R. van Tonder, A. Trockman, and C. L. Goues, "A panel data set of cryptocurrency development activity on GitHub," in Proceedings of the 16th International Conference on Mining Software Repositories, pp. 186-190, IEEE Press, 2019.

[110] Y. Zhauniarovich, Y. Boshmaf, H. A. Jawaheri, and M. A. Sabah, "Characterizing Bitcoin donations to open source software on GitHub," arXiv preprint arXiv:1907.04002, 2019.

[111] K. Blincoe, F. Harrison, and D. Damian, "Ecosystems in GitHub and a Method for Ecosystem Identification Using Reference Coupling," in 2015 IEEE/ACM 12th Working Conference on Mining Software Repositories, pp. 202-211, May 2015.

[112] Y. Zhang, D. Lo, P. S. Kochhar, X. Xia, Q. Li, and J. Sun, "Detecting similar repositories on GitHub," in 2017 IEEE 24th International Conference on Software Analysis, Evolution and Reengineering (SANER), pp. 13-23, Feb 2017.

[113] Z. Liao, N. Wang, S. Liu, Y. Zhang, H. Liu, and Q. Zhang, "IdentificationMethod Research for Open-Source Software Ecosystems," Symmetry, vol. 11, no. 2, p. 182, 2019.

[114] D. Spadini, M. Aniche, and A. Bacchelli, "Pydriller: Python framework for mining software repositories," in Proceedings of the 2018 26th ACM Joint Meeting on European Software Engineering Conference and Symposium on the Foundations of Software Engineering, pp. 908-911, 2018.

[115] "Google Big Query." https://cloud.google.com/bigquery/. Accessed: 2019-09-30.

[116] J. Ramos et al., "Using tf-idf to determine word relevance in document queries," in Proceedings of the first instructional conference on machine learning, vol. 242, pp. 133-142, New Jersey, USA, 2003.

[117] J. A. Hartigan and M. A. Wong, "Algorithm AS 136: A k-means clustering algorithm," Journal of the royal statistical society. series c (applied statistics), vol. 28, no. 1, pp. 100-108, 1979.

[118] T. Kohonen, "The self-organizing map," Proceedings of the IEEE, vol. 78, no. 9, pp. 1464-1480, 1990.

[119] E. A. Krasznai, P. Boda, A. Csercsa, M. Ficsor, and G. Varbiro, "Use of self organising maps in modelling the distribution patterns of gammarids (crustacea: Amphipoda)," Ecological Informatics, vol. 31, 112015. 
[120] T. M. Kodinariya and P. R. Makwana, "Review on determining number of Cluster in K-Means Clustering," International Journal, vol. 1, no. 6, pp. 90-95, 2013.

[121] P. J. Rousseeuw, "Silhouettes: a graphical aid to the interpretation and validation of cluster analysis," Journal of computational and applied mathematics, vol. 20, pp. 53-65, 1987.

[122] S. Basu, A. Banerjee, and R. Mooney, "Semi-supervised clustering by seeding," in In Proceedings of 19th International Conference on Machine Learning (ICML-2002, Citeseer, 2002.

[123] S. Basu, A. Banerjee, and R. J. Mooney, "Active semi-supervision for pairwise constrained clustering," in Proceedings of the 2004 SIAM international conference on data mining, pp. 333-344, SIAM, 2004.

[124] H. Borges and M. T. Valente, "What's in a GitHub star? understanding repository starring practices in a social coding platform," Journal of Systems and Software, vol. 146, pp. 112-129, 2018.

[125] "Cyclomatic complexity, author=Ebert, Christof and Cain, James and Antoniol, Giuliano and Counsell, Steve and Laplante, Phillip," IEEE software, vol. 33, no. 6, pp. 27-29, 2016.

[126] M. Bruntink and A. Van Deursen, "Predicting class testability using objectoriented metrics," in Source Code Analysis and Manipulation, Fourth IEEE International Workshop on, pp. 136-145, IEEE, 2004.

[127] T. J. McCabe, "A complexity measure," IEEE Transactions on software Engineering, no. 4, pp. 308-320, 1976.

[128] Y. U. Mshelia, "MECOT: a software quality metrics collection tool," Journal of Systems Integration, vol. 10, no. 1, pp. 21-35, 2019.

[129] T. Mccabe, "Cyclomatic complexity and the year 2000," IEEE Software, vol. 13, no. 3, pp. 115-117, 1996.

[130] P. Bellmann, P. Thiam, and F. Schwenker, "Using a Quartile-based Data Transformation for Pain Intensity Classification based on the SenseEmotion Database," in 2019 8th International Conference on Affective Computing and Intelligent Interaction Workshops and Demos (ACIIW), pp. 310-316, IEEE, 2019. 
[131] H.-C. Shih and E.-R. Liu, "New quartile-based region merging algorithm for unsupervised image segmentation using color-alone feature," Information Sciences, vol. 342, pp. 24-36, 2016.

[132] P. B. De Laat, "Governance of open source software: state of the art," Journal of Management \& Governance, vol. 11, no. 2, pp. 165-177, 2007.

[133] E. Alpaydin, Introduction to machine learning. MIT press, 2020.

[134] B. Schlkopf, A. J. Smola, and F. Bach, Learning with kernels: support vector machines, regularization, optimization, and beyond. the MIT Press, 2018.

[135] C. Lemaréchal, "Cauchy and the gradient method," Doc Math Extra, vol. 251, p. $254,2012$.

[136] M. Stone, "Cross-validatory choice and assessment of statistical predictions," Journal of the Royal Statistical Society: Series B (Methodological), vol. 36, no. 2, pp. 111-133, 1974.

[137] D. M. Powers, "Evaluation: from precision, recall and F-measure to ROC, informedness, markedness and correlation," 2011.

[138] C. Cortes and V. Vapnik, "Support-vector networks," Machine learning, vol. 20, no. 3, pp. 273-297, 1995.

[139] L. E. Peterson, "K-nearest neighbor," Scholarpedia, vol. 4, no. 2, p. 1883, 2009.

[140] L. Rokach and O. Z. Maimon, Data mining with decision trees: theory and applications, vol. 69. World scientific, 2008.

[141] I. Rish et al., "An empirical study of the naive Bayes classifier," in IJCAI 2001 workshop on empirical methods in artificial intelligence, vol. 3, pp. 41-46, 2001.

[142] "Eli5-scikit-learn." https://eli5.readthedocs.io/en/latest/libraries/ sklearn.html. Accessed: 2019-09-30.

[143] A. Altmann, L. Toloşi, O. Sander, and T. Lengauer, "Permutation importance: a corrected feature importance measure," Bioinformatics, vol. 26, no. 10, pp. 1340-1347, 2010.

[144] "GitHub." https://github.com/terryyin/lizard. Accessed: 2019-09-30.

[145] "Scikit-learn." https://scikit-learn.org/stable/. Accessed: 2019-09-30. 
[146] "Pandas." https://pandas.pydata.org/. Accessed: 2019-09-30.

[147] "Numpy." https://numpy.org/. Accessed: 2019-09-30.

[148] "Seaborn." https://seaborn.pydata.org/. Accessed: 2019-09-30.

[149] "Google Colaboratory." https://colab.research.google.com/notebooks/ intro.ipynb. Accessed: 2019-09-30.

[150] "Bitcoin Core." https://github.com/bitcoin/bitcoin. Accessed: 2019-0930.

[151] "CCTX." https://github.com/ccxt/ccxt. Accessed: 2019-09-30.

[152] "bitcoin security threat model." https://github.com/JWWeatherman/ bitcoin_security_threat_model. Accessed: 2019-09-30.

[153] "Bitcoin Visuals." https://bitcoinvisuals.com/. Accessed: 2019-09-30.

[154] "Coin Dance, howpublished $=$ https://coin.dance/, note = Accessed: 201909-30."

[155] "Blockstream." https://blockstream.com/. Accessed: 2019-09-30.

[156] "OpenBazaar Client v2." https://github.com/OpenBazaar/ openbazaar-desktop. Accessed: 2019-09-30.

[157] "Casa." https://keys.casa/node-features/. Accessed: 2019-09-30.

[158] "Bitcoin Lightning." https://bitcoinlightning.com/ what-is-bitcoin-lightning/. Accessed: 2019-09-30.

[159] "eclair." https://github.com/ACINQ/eclair. Accessed: 2019-09-30.

[160] "Electrum." https://github.com/spesmilo/electrum. Accessed: 2019-0930.

[161] "bither-android." https://github.com/bither/bither-android. Accessed: 2019-09-30.

[162] "BTCMiner for Bitcoin." https://en.bitcoin.it/wiki/BTCMiner. Accessed: 2019-09-30.

[163] "Oxbitcoin." https://github.com/0xbitcoin/Oxbitcoin-miner. Accessed: 2019-09-30. 
[164] "Metatrader5." https://www.metatrader5.com/en. Accessed: 2019-09-30.

[165] "zenbot." https://github.com/DeviaVir/zenbot. Accessed: 2019-09-30.

[166] "twitterbitcoin." https://github.com/joelsonderegger/twitterbitcoin. Accessed: 2019-09-30.

[167] "BTCPay Server." https://en.bitcoin.it/wiki/BTCPay, Accessed: 201909-30.

[168] D. Wahyudin, K. Mustofa, A. Schatten, S. Biffl, and A. M. Tjoa, "Monitoring the "health" status of open source web-engineering projects," International Journal of Web Information Systems, 2007.

[169] E. Constantinou and T. Mens, "An empirical comparison of developer retention in the rubygems and npm software ecosystems," Innovations in Systems and Software Engineering, vol. 13, no. 2-3, pp. 101-115, 2017.

[170] B. Lin, G. Robles, and A. Serebrenik, "Developer turnover in global, industrial open source projects: Insights from applying survival analysis," in 2017 IEEE 12th International Conference on Global Software Engineering (ICGSE), pp. 66-75, IEEE, 2017.

[171] V. Midha and P. Palvia, "Retention and quality in open source software projects," AMCIS 2007 Proceedings, p. 25, 2007.

[172] R. v. W. de Joode and M. de Bruijne, "The organization of open source communities: Towards a Framework to Analyze the relationship between openness and reliability," in Proceedings of the 39th Annual Hawaii International Conference on System Sciences (HICSS'06), vol. 6, pp. 118b-118b, IEEE, 2006.

[173] "Eli5-scikit-learn." https://www.washingtonpost.com/nation/2019/12/16/ gerald-cotten-quadrigacx-cryptocurrency-death-body-exhumed/. Accessed: 2019-09-30.

[174] F. Mai, Z. Shan, Q. Bai, X. Wang, and R. H. Chiang, "How does social media impact bitcoin value? a test of the silent majority hypothesis," Journal of Management Information Systems, vol. 35, no. 1, pp. 19-52, 2018.

[175] P. Xie, J. Wu, and C. Wu, "Social data predictive power comparison across information channels and user groups: evidence from the bitcoin market," The Journal of Business Inquiry, vol. 17, no. 1, pp. 41-54, 2017. 
[176] M. Al Guindy and R. Riordan, "Tweeting the good news: Returns and price informativeness," Available at SSRN 2999443, 2017.

[177] M. Al Guindy and R. Riordan, "The social internetwork and stock returns," Available at SSRN 3501915, 2019.

[178] A. M. Balfagih and V. Keselj, "Evaluating sentiment classifiers for bitcoin tweets in price prediction task," in 2019 IEEE International Conference on Big Data (Big Data), pp. 5499-5506, IEEE, 2019. 